KEK-TH-1537

RIKEN-QHP-17

\title{
Non-Abelian statistics of vortices with non-Abelian Dirac fermions
}

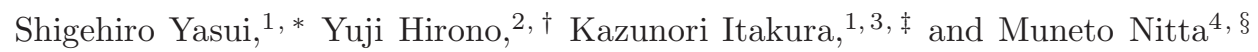 \\ ${ }^{1}$ KEK Theory Center, Institute of Particle and Nuclear Studies, \\ High Energy Accelerator Research Organization (KEK), 1-1 Oho, Tsukuba, Ibaraki 305-0801, Japan \\ ${ }_{2}$ Department of Physics, University of Tokyo, Hongo 7-3-1, Bunkyo-ku, Tokyo 113-0033, Japan \\ ${ }^{3}$ Department of Particle and Nuclear Studies, Graduate University for \\ Advanced Studies (SOKENDAI), 1-1 Oho, Tsukuba, Ibaraki 305-0801, Japan \\ ${ }^{4}$ Department of Physics, and Research and Education Center for Natural Sciences, \\ Keio University, 4-1-1 Hiyoshi, Yokohama, Kanagawa 223-8521, Japan
}

(Dated: February 28, 2018)

\begin{abstract}
We extend our previous analysis on the exchange statistics of vortices having a single Dirac fermion trapped in each core, to the case where vortices trap two Dirac fermions with U(2) symmetry. Such a system of vortices with non-Abelian Dirac fermions appears in color superconductors at extremely high densities, and in supersymmetric QCD. We show that the exchange of two vortices having doublet Dirac fermions in each core is expressed by non-Abelian representations of a braid group, which is explicitly verified in the matrix representation of the exchange operators when the number of vortices is up to four. We find that the result contains the matrices previously obtained for the vortices with a single Dirac fermion in each core as a special case. The whole braid group does not immediately imply non-Abelian statistics of identical particles because it also contains exchanges between vortices with different numbers of Dirac fermions. However, we find that it does contain, as its subgroup, genuine non-Abelian statistics for the exchange of the identical particles, that is, vortices with the same number of Dirac fermions. This result is surprising compared with conventional understanding because all Dirac fermions are defined locally at each vortex, unlike the case of Majorana fermions for which Dirac fermions are defined non-locally by Majorana fermions located at two spatially separated vortices.
\end{abstract}

PACS numbers: 05.30.Pr, 74.25.Uv, 67.85.-d, 21.65.Qr, 03.67.-a

\section{INTRODUCTION}

Topological insulators/superconductors have an attractive property that some of them possess quantum vortices which trap zero-energy, Majorana or Dirac, fermions in their cores 1, 2]. The existence of such zero-energy fermions is topologically protected and is robust against small perturbations [3, 4]. Thus, when we consider adiabatic manipulation of vortices such as interchanging the positions of two vortices, we can treat the vortices as objects that are always accompanied by zero-energy fermions. In particular, according to the recent discoveries, the exchange of such vortices can be represented by a non-trivial representation of a braid group, whose precise form is determined by the trapped zero-energy Majorana fermions [5 [10] and Dirac fermions [11]. The exchange of vortices with Majorana fermions gives non-Abelian statistics because they are all identical particles. The statistics is called non-Abelian because quantum states of two vortices transform non-diagonally under the exchange of two vortices (i.e., the exchange operation is described by non-diagonal matrices acting on the quantum states), and two adjacent exchange operations (such as those for vortex pairs $(1,2)$ and $(2,3))$ do not commute with each other. This is highly contrasted with the ordinary statistics where only a phase factor $\mathrm{e}^{i \theta}$ appears under the exchange of two particles $(\theta=0$ for the Bose-Einstein, $\theta=\pi$ for the Fermi-Dirac, and others for the anyon statistics), and two adjacent operations are commutative. On the other hand, it is unclear whether the exchange of vortices with Dirac fermions gives a non-Abelian statistics or not

\footnotetext{
*Electronic address: yasuis@post.kek.jp

${ }^{\dagger}$ Electronic address: hirono@nt.phys.s.u-tokyo.ac.jp

¥Electronic address: kazunori.itakura@kek.jp

$\S$ Electronic address: nitta@phys-h.keio.ac.jp
} 
because, in general, it exchanges different particles, that is, vortices with different numbers of Dirac fermions. For U(1) Dirac fermions, vortices can be distinguished by the occupancy of Dirac fermions.

According to the classification of topological insulators/superconductors [1, 2] and its extension to the case with topological defects [4, 12], the vortices with the Majorana or Dirac fermions are categorized into different types: class D for the Majorana and classes C and DIII for the Dirac. However, the essential difference between the vortices with Majorana fermions and Dirac fermions is the parity of the number of zero-energy Majorana fermions trapped to a single vortex. Notice that a single Dirac fermion corresponds to two Majorana fermions. Thus, when a vortex traps an even number of the Majorana fermions, it should be regarded as the Dirac case, while an odd number of the Majorana fermions, just as the Majorana case [4]. So far, non-Abelian unitary transformations (non-Abelian representations of a braid group) are found both in the Majorana and Dirac cases. In the Majorana case, the non-Abelian statistics was first discovered when a vortex has a single Majorana fermion [ [ ] $]$ and later when a vortex can have multiple Majorana fermions with non-Abelian symmetry [8 10]. In contrast, in the Dirac case, while non-Abelian representation of a braid group is analyzed when a vortex traps a single Dirac fermion [11], it is unclear if it gives genuine non-Abelian statistics. 229] The present paper discusses the non-Abelian representation of a braid group for the exchange of vortices which have multiple Dirac fermions with non-Abelian symmetry, to complete the series of analyses. We then show that it indeed contains genuine non-Abelian statistics of the exchanges of identical particles, that is, vortices with the same numbers of Dirac fermions. This result is somewhat surprising compared with conventional understanding because non-Abelian statistics appears in a system with only locally-defined Dirac fermions.

There is an important difference between the vortices with Majorana fermions and Dirac fermions. Consider a system of vortices each of which traps only a single Majorana fermion. Recall that the Majorana fermion has a unique property that there is no distinction between a particle and a hole (anti-particle) [13]. Thus, in order to define the Fock vacuum, one has to introduce a Dirac fermion by using two Majorana fermions that belong to different vortices [5, 6]. Therefore, the Dirac fermions thus constructed are non-local objects. When there are $2 m$ vortices, the total Hilbert space of the zero-energy fermions has a dimension (degeneracy) of $2^{m}$, where each zero-energy Dirac fermion has the dimension 2 (empty or occupied). On the other hand, in the system of vortices each of which traps a single Dirac fermion, we can immediately construct the Hilbert space without introducing 'non-local' Dirac fermions [11], and find a non-Abelian representation of a braid group (but not non-Abelian statistics) for the exchange of two vortices. When there are $2 m$ vortices, the dimension of the Hilbert space is $2^{2 m}=4^{m}$.

In the Majorana case, increasing the number of Majorana fermions in a single vortex brings in an interesting nontrivial structure. When the multiple Majorana fermions are in the vector representations of $\mathrm{SO}(3)$ [ 8 ] and, in general, $\mathrm{SO}(2 N-1)$ [10], the exchange matrices of two vortices are given as tensor products of the matrices that appear in the single Majorana case (called the Ivanov matrices) and generators of the Coxeter group of the $A_{2 m-1}$ type (for $2 m$ vortices). Besides, the dimension of the Hilbert space of zero-energy fermions becomes larger than that of the single Majorana case by the internal degrees of freedom. For example, when three (triplet) Majorana fermions with $\mathrm{SO}(3)$ symmetry are trapped, the dimension is $2^{3 m}$ which should be compared with $2^{m}$ for the case with a single Majorana fermion. It is not known if a similar structure exists and how the dimension of the Hilbert space is enlarged, when increasing the number of Dirac fermions at each vortex. The purpose of the present paper is to show the explicit forms of the non-Abelian representation of a braid group for vortices with multiple Dirac fermions, and that it contains genuine non-Abelian statistics in the sectors of the exchange of vortices with the same numbers of Dirac fermions. As the simplest but non-trivial example, we focus on the Dirac fermions with U(2) symmetry. Extension to general cases will become more complicated, but should be straightforward.

It should be noticed that, since the vortices with zero-energy fermions are characterized by topology, they appear in many different quantum systems. For example, vortices with the Majorana fermions are realized in chiral $p$-wave superconductors [3], such as $\mathrm{Sr}_{2} \mathrm{RuO}_{4}$ [14], in chiral $p$-wave superfluids, such as the A-phase of ${ }^{3} \mathrm{He}$ in $2+1$ dimensions, and also in other systems [15, 16]. Vortices with the Dirac fermions are identified with the integer (singular) vortices in the ${ }^{3} \mathrm{He}$ A-phase in $2+1$ dimensions [17] the normal " $o$ " vortices in the ${ }^{3} \mathrm{He} \mathrm{B}$-phase in $3+1$ dimensions [18] and also with dislocation lines in topological insulators [19]. In these examples, the vortex has only a single zero-energy Majorana or Dirac fermion at its core. So far, there is no condensed-matter example of vortices with more than two Majorana fermions. However, we know at least two examples in high-energy physics. In fact, the existence of zero-energy modes in the vortex-fermion system was discussed long time ago in the context of relativistic quantum field theory [20].

The primary example is the color superconductor in QCD which could exist in extremely high density matter such as in the cores of neutron stars [21]. In particular, vortices with non-Abelian symmetries appear in the color-flavor locked (CFL) phase where the original color $\mathrm{SU}(3)$ and flavor $\mathrm{SU}(3)$ symmetry in the vacuum breaks down to $\mathrm{SU}(3)$ with color and flavor degrees are locked [22]. The SU(3) CFL symmetry is further broken down to a U(2) symmetry in the core of non-Abelian vortices [23]. It has been recently shown [24] by using the index theorem that there appear two types of non-Abelian vortices: the vortex which traps a triplet of zero-energy Majorana fermions [9] and the vortex which traps an $\mathrm{U}(2)$ doublet of zero-energy Dirac fermions. While the former gives an example of non-Abelian 
statistics of vortices with multiple Majorana fermions [8], the latter gives an example of non-Abelian representation of a braid group for the exchange of vortices with doublet Dirac fermions focused in the present paper. The secondary example is the non-Abelian vortices in supersymmetric QCD with $\mathrm{U}(N)$ gauge symmetry [25] (see Ref. [26] for reviews). In this case, the color $\mathrm{U}(N)$ symmetry and the flavor $\mathrm{SU}(N)$ symmetry are spontaneously broken down to the $\mathrm{SU}(N)$ color-flavor locked symmetry by the scalar quark condensates in the vacuum, and it is further broken down to a $\mathrm{U}(N-1)$ symmetry in the core of non-Abelian vortices. These vortices contain one singlet and one $(N-1)$-plet of zero-energy Dirac fermions in their core. Therefore, it gives an example of non-Abelian statistics of vortices with arbitrary number of Dirac fermions.

Before finishing Introduction, let us briefly comment on the potential application to quantum computers [27, 28]. As discussed above, the system of vortices with zero-energy fermions is robust against small perturbations from environment, and has the Hilbert space with a large dimension. These are desirable properties as quantum computers. Comparing the Majorana and Dirac cases, it should be noticed that the Dirac case is simpler because we do not have to introduce non-local Dirac fermions. Since vortex systems with non-Abelian symmetry have larger dimensions, it would be worth to consider the case with non-Abelian Dirac fermions, even though it has not been realized in laboratory.

This paper is organized as follows. In Section II we briefly summarize the non-Abelian statistics for the vortices trapping a single Dirac fermion with U(1) symmetry at each core, as presented in Ref. [11]. In Section [II, we discuss the non-Abelian representation of the braid group for the exchange of the vortices trapping doublet Dirac fermions with U(2) symmetry. In Section IV we discuss the difference between non-Abelian representations of the braid group for the exchange of the Dirac fermion with $\mathrm{U}(1)$ symmetry and that with $\mathrm{U}(2)$ symmetry. In Section $\mathrm{V}$ we show that the whole braid group contains genuine non-Abelian statistics as its subgroup, in the sectors of the vortices with the same number of Dirac fermions. Section VI is devoted to a summary. In Appendices, we present detailed supplementary information. In Appendix $\mathrm{A}$ we give the transformation matrices for U(1) (singlet) Dirac fermions. In Appendix B we give the Hilbert space and the exchange matrices for $n=4 \mathrm{U}(2)$ Dirac vortices. In Appendix C. we discuss restricted Hilbert subspaces, in which two successive exchanges of vortices is equal to the identity.

\section{NON-ABELIAN REPRESENTATION OF BRAID GROUP FOR EXCHANGE OF U(1) DIRAC VORTICES}

Let us first explain how the non-Abelian representation of the braid group appears in the system of vortices having a single Dirac fermion in each core, which corresponds to considering the Dirac fermion with the U(1) symmetry. This is a brief summary of the recent work done by three of us [1]. We highlight the similarities to and differences from the case with Majorana fermions. Below, we call vortices with the Dirac fermions "the Dirac vortices", while vortices with the Majorana fermions, "the Majorana vortices".

Consider $n$ Dirac vortices which are labelled by $k=1, \cdots, n$. The number of vortices, $n$, can be arbitrary in contrast with the case of Majorana vortices where we define Dirac fermions by using two Majorana fermions, and thus the total fermion number is even. The operator $\hat{\psi}_{k}$ denotes the Dirac fermion of the $k$-th vortex. Together with its hermitian conjugate $\hat{\psi}_{k}^{\dagger}$, they satisfy the following algebra:

$$
\left\{\hat{\psi}_{k}, \hat{\psi}_{\ell}^{\dagger}\right\}=\delta_{k \ell}, \quad\left\{\hat{\psi}_{k}, \hat{\psi}_{\ell}\right\}=0, \quad\left\{\hat{\psi}_{k}^{\dagger}, \hat{\psi}_{\ell}^{\dagger}\right\}=0 .
$$

We regard $\hat{\psi}_{k}$ and $\hat{\psi}_{k}^{\dagger}$ as the annihilation and creation operators, respectively. Exchange of the $k$-th and $(k+1)$-th vortices, which is denoted by $T_{k}$, induces the following exchange of $\hat{\psi}_{k}$ and $\hat{\psi}_{k+1}$,

$$
T_{k}:\left\{\begin{array}{l}
\hat{\psi}_{k} \rightarrow \hat{\psi}_{k+1} \\
\hat{\psi}_{k+1} \rightarrow-\hat{\psi}_{k}
\end{array}\right.
$$

with the rest $\hat{\psi}_{\ell}(\ell \neq k$ and $k+1)$ unchanged. We note that $T_{k}$ 's satisfy the braid relations,

$$
\begin{aligned}
& \text { (i) } T_{k} T_{\ell} T_{k}=T_{\ell} T_{k} T_{\ell} \quad \text { for } \quad|k-\ell|=1, \\
& \text { (ii) } T_{k} T_{\ell}=T_{\ell} T_{k} \quad \text { for } \quad|k-\ell|>1,
\end{aligned}
$$

as a general rule of exchange operations. Notice that these relations and the transformation (2.2) are the same as in the case of the Majorana vortices, while the fermion operators $\hat{\psi}_{k}$ and $\hat{\psi}_{k}^{\dagger}$ now satisfy Eq. (2.1), instead of the Clifford algebra $\left\{\hat{\gamma}_{k}, \hat{\gamma}_{\ell}\right\}=2 \delta_{k \ell}$ satisfied by the Majorana fermion operators $\hat{\gamma}_{k}$ 's. Therefore, one has the same property of the operator $T_{k}$, namely, four-time successive application of $T_{k}$ is equivalent to the identity, $\left(T_{k}\right)^{4}=1$. This fact by itself suggests that the exchange of these vortices shows a representation of the braid group different from the ordinary 
Bose-Einstein, Fermi-Dirac or Abelian anyon one which shows Abelian representation of the braid group. In fact, the exchange statistics of the Majorana vortices showing the same property $\left(T_{k}\right)^{4}=1$ turned out to be non-Abelian [6, 8, 10], and we will discuss below if the same is true for the Dirac vortices.

The transformation (2.2) can be represented by the following unitary operator

$$
\hat{\tau}_{k}^{\mathrm{s}} \equiv 1+\hat{\psi}_{k+1} \hat{\psi}_{k}^{\dagger}+\hat{\psi}_{k+1}^{\dagger} \hat{\psi}_{k}-\hat{\psi}_{k+1}^{\dagger} \hat{\psi}_{k+1}-\hat{\psi}_{k}^{\dagger} \hat{\psi}_{k}+2 \hat{\psi}_{k+1}^{\dagger} \hat{\psi}_{k+1} \hat{\psi}_{k}^{\dagger} \hat{\psi}_{k}
$$

so that $\hat{\tau}_{k}^{\mathrm{s}} \hat{\psi}_{\ell}\left(\hat{\tau}_{k}^{\mathrm{s}}\right)^{-1}(\ell=1, \cdots, n)$ reproduces the transformation law. The superscript "s" implies the singlet Dirac fermion. One can also confirm by a straightforward calculation that $\hat{\tau}_{k}$ 's satisfy the braid relations,

$$
\begin{aligned}
& \text { (i') } \hat{\tau}_{k}^{\mathrm{s}} \hat{\tau}_{\ell}^{\mathrm{s}} \hat{\tau}_{k}^{\mathrm{s}}=\hat{\tau}_{\ell}^{\mathrm{s}} \hat{\tau}_{k}^{\mathrm{s}} \hat{\tau}_{\ell}^{\mathrm{s}} \quad \text { for } \quad|k-\ell|=1, \\
& \text { (ii') } \hat{\tau}_{k}^{\mathrm{s}} \hat{\tau}_{\ell}^{\mathrm{s}}=\hat{\tau}_{\ell}^{\mathrm{s}} \hat{\tau}_{k}^{\mathrm{s}} \quad \text { for } \quad|k-\ell|>1 .
\end{aligned}
$$

Having the explicit form of the exchange operator (2.5), we are able to check the representation of the braid group for the exchange of the Dirac vortices. First of all, as we mentioned above, four successive exchanges of the $k$-th and $(k+1)$-th vortices indeed yield the identity:

$$
\left(\hat{\tau}_{k}^{\mathrm{s}}\right)^{4}=1
$$

while two successive exchanges do not,

$$
\left(\hat{\tau}_{k}^{\mathrm{s}}\right)^{2}=\left(1-2 \hat{\psi}_{k}^{\dagger} \hat{\psi}_{k}\right)\left(1-2 \hat{\psi}_{k+1}^{\dagger} \hat{\psi}_{k+1}\right) \neq 1
$$

Next, it should be noted that $\hat{\tau}_{k}^{\mathrm{s}}$ and $\hat{\tau}_{k+1}^{\mathrm{s}}$ are not commutative;

$$
\left[\hat{\tau}_{k}^{\mathrm{s}}, \hat{\tau}_{k+1}^{\mathrm{s}}\right] \neq 0
$$

Therefore, one observes at the operator level that the exchange operation of two Dirac vortices is in general nonAbelian. However, this observation does not necessarily mean that the exchange is always non-Abelian. In order to confirm whether the exchange operation is indeed non-Abelian, we have to check if the commutator $\left[\hat{\tau}_{k}^{\mathrm{s}}, \hat{\tau}_{k+1}^{\mathrm{s}}\right]$ does not vanish in the matrix representation.

Let us consider the matrix representation of the operator $\hat{\tau}_{k}^{\mathrm{s}}$. As a basis of the Hilbert space, we choose the Fock states defined by the Dirac fermion operators $\hat{\psi}_{k}$ 's. One of the merits of the Dirac vortices is that we can construct the Hilbert space naturally by using locally-defined Dirac fermions, which is in clear contrast with the Majorana case. We first define the Fock vacuum state $|0\rangle$ by

$$
\hat{\psi}_{\ell}|0\rangle=0 \text { for all } \ell \text {. }
$$

Then, by acting successively the creation operators $\hat{\psi}_{\ell}^{\dagger}$ 's on the vacuum, we obtain the $f$-particle state $(0 \leq f \leq n)$

$$
\begin{aligned}
& |0 \cdots 01 \cdots 1 \cdots 10 \cdots 0\rangle=\hat{\psi}_{\ell_{1}}^{\dagger} \ldots \hat{\psi}_{\ell_{i}}^{\dagger} \ldots \hat{\psi}_{\ell_{f}}^{\dagger}|0\rangle, \\
& \check{1} \quad \check{\ell}_{1} \quad \check{\ell}_{i} \quad \check{\ell}_{f} \quad \check{n}
\end{aligned}
$$

in which the $\ell_{i}$-th $(i=1, \cdots, f)$ vortex is occupied by a Dirac fermion, while the other vortices are empty.

When we have only one vortex, $n=1$ ( $k$-th vortex), there are two Fock states, $|0\rangle$ and $|1\rangle \equiv \hat{\psi}_{k}^{\dagger}|0\rangle$.

When we have two vortices, $n=2\left(k\right.$-th and $(k+1)$-th vortices), there are $2^{2}=4$ Fock states, $|00\rangle \equiv|0\rangle$, $|10\rangle \equiv \hat{\psi}_{k}^{\dagger}|0\rangle,|01\rangle \equiv \hat{\psi}_{k+1}^{\dagger}|0\rangle$ and $|11\rangle \equiv \hat{\psi}_{k}^{\dagger} \hat{\psi}_{k+1}^{\dagger}|0\rangle$.

We can similarly obtain the Fock states for any number of vortices $n$. The basis of the whole Hilbert space for the $n$-vortex system is given by a tensor product of the 4 Fock states constructed at each vortex. Because the fermion number operator $\hat{f}^{\mathrm{s}} \equiv \sum_{i=1}^{n} \hat{\psi}_{i}^{\dagger} \hat{\psi}_{i}$ commutes with $\hat{\tau}_{\ell}^{\mathrm{s}}$ for $\ell=1, \cdots, n-1$, the fermion number $f$ (an eigenvalue of $\left.\hat{f}^{\mathrm{s}}\right)$ is a conserved quantity under the exchange of vortices. Thus, the whole Hilbert space $\mathbb{H}^{(n)}$ for $n$ vortices (with the dimension $2^{n}$ ) can be decomposed into subspaces $\mathbb{H}^{(n, f)}$ labelled further by the total fermion number $f$ : $\mathbb{H}^{(n)}=\oplus_{f=1}^{n} \mathbb{H}^{(n, f)}$. Then, in each subspace, the operators $\hat{\tau}_{\ell}^{\mathrm{s}}$ 's are represented by matrices, whose explicit expressions are shown up to $n=4$ in Appendix $\mathrm{A}$. One can confirm that the matrix representations of the exchange operators $\hat{\tau}_{\ell}$ 's are indeed non-Abelian for $n \geq 3$. 


\section{NON-ABELIAN REPRESENTATION OF BRAID GROUP FOR EXCHANGE OF U(2) DIRAC VORTICES}

Now let us turn to the case with vortices which trap two massless Dirac fermions having the "pseudo-spin" U(2) symmetry. In particular, we consider Dirac fermions in the doublet of $\mathrm{U}(2)$, which are denoted by $\hat{\psi}_{k}^{a}(a=1,2)$ for the $k$-th vortex. We also use the vector notation for the doublet $\left(\hat{\psi}_{k}^{1}, \hat{\psi}_{k}^{2}\right)^{\mathrm{t}}$. The exchange of the $k$-th and $(k+1)$-th vortices induces transformation of the Dirac fermions. Here we consider the simplest transformation similarly as the single Dirac fermion shown in Eq. (2.2):

$$
T_{k}:\left\{\begin{array}{l}
\hat{\psi}_{k}^{a} \rightarrow \hat{\psi}_{k+1}^{a} \\
\hat{\psi}_{k+1}^{a} \rightarrow-\hat{\psi}_{k}^{a}
\end{array}\right.
$$

for each $a=1,2$, with the rest $\hat{\psi}_{\ell}^{a}(\ell \neq k$ and $k+1)$ unchanged. This transformation is expressed by the unitary operator

$$
\hat{\tau}_{k} \equiv \prod_{a=1,2}\left(1+\hat{\psi}_{k+1}^{a} \hat{\psi}_{k}^{a \dagger}+\hat{\psi}_{k+1}^{a \dagger} \hat{\psi}_{k}^{a}-\hat{\psi}_{k+1}^{a \dagger} \hat{\psi}_{k+1}^{a}-\hat{\psi}_{k}^{a \dagger} \hat{\psi}_{k}^{a}+2 \hat{\psi}_{k+1}^{a \dagger} \hat{\psi}_{k+1}^{a} \hat{\psi}_{k}^{a \dagger} \hat{\psi}_{k}^{a}\right)
$$

which is invariant under the $\mathrm{U}(2)$ transformation, $\left(\hat{\psi}_{k}^{1}, \hat{\psi}_{k}^{2}\right)^{\mathrm{t}} \rightarrow \exp (i \varphi) \exp (i \vec{\theta} \cdot \vec{\sigma} / 2)\left(\hat{\psi}_{k}^{1}, \hat{\psi}_{k}^{2}\right)^{\mathrm{t}}$ with $\varphi$ and $\vec{\theta}$ being parameters and $\vec{\sigma}$ the Pauli matrices. We confirm that $\hat{\tau}_{k} \hat{\psi}_{\ell}^{a} \hat{\tau}_{k}^{-1}(a=1,2)$ reproduces the transformation (3.1), and that $\hat{\tau}_{k}$ 's satisfy the braid relations, (i') $\hat{\tau}_{k} \hat{\tau}_{\ell} \hat{\tau}_{k}=\hat{\tau}_{\ell} \hat{\tau}_{k} \hat{\tau}_{\ell}$ for $|k-\ell|=1$ and (ii') $\hat{\tau}_{k} \hat{\tau}_{\ell}=\hat{\tau}_{\ell} \hat{\tau}_{k}$ for $|k-\ell|>1$. Similarly as in the U(1) case (see Eqs. (2.8) and (2.9)), the exchange operator $\hat{\tau}_{k}$ satisfies

$$
\left(\hat{\tau}_{k}\right)^{4}=1
$$

while two successive exchanges do not go back to the identity at the operator level:

$$
\left(\hat{\tau}_{k}\right)^{2}=\left(1-2 \hat{\psi}_{k}^{1 \dagger} \hat{\psi}_{k}^{1}\right)\left(1-2 \hat{\psi}_{k}^{2 \dagger} \hat{\psi}_{k}^{2}\right)\left(1-2 \hat{\psi}_{k+1}^{1 \dagger} \hat{\psi}_{k+1}^{1}\right)\left(1-2 \hat{\psi}_{k+1}^{2 \dagger} \hat{\psi}_{k+1}^{2}\right) \neq 1 \text {. }
$$

We can also check that $\hat{\tau}_{k}$ and $\hat{\tau}_{k+1}$ are non-commutative, which suggests that the representation of the braid group for the exchange of $\mathrm{U}(2)$ Dirac vortices is non-Abelian.

We define the number operator of the Dirac fermions

$$
\hat{f}=\sum_{\ell=1}^{n} \sum_{a=1,2} \hat{\psi}_{\ell}^{a \dagger} \hat{\psi}_{\ell}^{a}
$$

whose eigenvalues $f$ give the total number of zero-energy Dirac fermions in the vortex system $(0 \leq f \leq 2 n)$. We note that $\sum_{a=1,2} \psi_{k}^{a \dagger} \psi_{k}^{a}$ is $\mathrm{U}(2)$ invariant, hence $\hat{f}$ is also $\mathrm{U}(2)$ invariant. We also note that $\hat{f}$ commutes with $\hat{\tau}_{\ell}$ for $\ell=1, \cdots, n-1$, hence $f$ is a conserved quantity under the exchange of two Dirac vortices.

We can construct the Hilbert space by successively applying $\hat{\psi}_{\ell}^{a \dagger}$ to the Fock vacuum $|0\rangle$ defined by

$$
\hat{\psi}_{\ell}^{a}|0\rangle=0 \text { for all } \ell \text { and } a=1,2
$$

Below, we explicitly construct the Hilbert spaces when the numbers of vortices are $n=1,2,3$, and see the non-Abelian properties of the exchange operation in the matrix representations for $n=2$ and 3 . The results for $n=4$ vortices are quite involved and are shown in Appendix B.

\section{A. The case of $n=1$}

When we have only one vortex, we are not able to discuss the exchange of vortices. However, let us consider this case to demonstrate how to construct the Hilbert space for the U(2) Dirac vortex. We have two massless Dirac fermion operators $\hat{\psi}_{k}^{1}$ and $\hat{\psi}_{k}^{2}$ (to avoid notational confusion, we use the label $k$ to specify this single vortex). By applying the creation operators $\hat{\psi}_{k}^{1 \dagger}$ and $\hat{\psi}_{k}^{2 \dagger}$ to the Fock vacuum $|0\rangle$, we obtain $2 \times 2=4$ energetically degenerate states (2 for empty/occupied, and another 2 for $a=1,2)$. Let us introduce the notation $\left|\mathcal{R}_{f}\right\rangle$ where $\mathcal{R}$ is the representation of $\mathrm{U}(2)$ group and $f$ is the total number of the Dirac fermions. Namely,

$$
\left|\mathbf{1}_{0}\right\rangle \equiv|0\rangle,
$$




$$
\begin{aligned}
&\left|\mathbf{2}_{1}\right\rangle \equiv\left(\begin{array}{c}
\hat{\psi}_{k}^{1 \dagger} \\
\hat{\psi}_{k}^{2 \dagger}
\end{array}\right)|0\rangle, \\
&\left|\mathbf{1}_{2}\right\rangle \equiv \hat{\psi}_{k}^{1 \dagger} \hat{\psi}_{k}^{2 \dagger}|0\rangle .
\end{aligned}
$$

The bold numbers 1, 2 imply the singlet and doublet representations of the U(2) group. Notice that the fully occupied state $\hat{\psi}_{k}^{1 \dagger} \hat{\psi}_{k}^{2 \dagger}|0\rangle$ is invariant under the $\mathrm{U}(2)$ transformation, thus it belongs to the singlet representation. Hence, there are two singlet states $\left|\mathbf{1}_{0}\right\rangle$ (empty) and $\left|\mathbf{1}_{2}\right\rangle$ (fully-occupied by two fermions), and one doublet state $\left|\mathbf{2}_{1}\right\rangle$ (occupied by one fermion). We use these states as the basis to span the whole Hilbert space of the zero-energy states. Therefore, we have decomposed the representations of the $\mathrm{U}(2)$ pseudo-spin as

$$
\mathbf{1}_{0}+\mathbf{2}_{1}+\mathbf{1}_{2},
$$

and correspondingly the whole Hilbert space of a single vortex $\mathbb{H}^{\{n=1\}}$ into a direct sum

$$
\mathbb{H}^{\{n=1\}}=\mathbb{H}^{\mathbf{1}_{[0]}} \oplus \mathbb{H}^{\mathbf{2}_{[1]}} \oplus \mathbb{H}^{\mathbf{1}_{[2]},}
$$

where $\mathbb{H}^{\mathbf{1}_{[0]}} \equiv\left\{\left|\mathbf{1}_{0}\right\rangle\right\}, \mathbb{H}^{\mathbf{2}_{[1]}} \equiv\left\{\left|\mathbf{2}_{1}\right\rangle\right\}$ and $\mathbb{H}^{\mathbf{1}_{[2]}} \equiv\left\{\left|\mathbf{1}_{2}\right\rangle\right\}$.

\section{B. The case of $n=2$}

Consider the case when we have two Dirac vortices which are respectively labelled by $k$ and $k+1$. First of all, we can use the decomposition for a single Dirac vortex, Eq. (3.8), to decompose the whole Hilbert space of two Dirac vortices into representations. Since each vortex contains the representations $\left(\mathbf{1}_{0}+\mathbf{2}_{1}+\mathbf{1}_{2}\right)_{\text {vortex } \ell}(\ell=k, k+1)$, the total representation is obtained as a tensor product of them, which can be decomposed as

$$
\begin{aligned}
\left(\mathbf{1}_{0}+\mathbf{2}_{1}\right. & \left.+\mathbf{1}_{2}\right)_{\text {vortex } k} \otimes\left(\mathbf{1}_{0}+\mathbf{2}_{1}+\mathbf{1}_{2}\right)_{\text {vortex } k+1} \\
& =\mathbf{1}_{00}+\mathbf{1}_{11}+\mathbf{1}_{20}+\mathbf{1}_{02}+\mathbf{1}_{22} \\
& +\mathbf{2}_{10}+\mathbf{2}_{01}+\mathbf{2}_{21}+\mathbf{2}_{12} \\
& +\mathbf{3}_{11}
\end{aligned}
$$

where the bold numbers denote representations, and the subscript $n_{k} n_{k+1}\left(n_{k}, n_{k+1}=0,1,2\right)$ denotes the number of the Dirac fermions, $n_{k}$ and $n_{k+1}$, at the $k$-th and $(k+1)$-th vortices, respectively. Next, one obtains the basis of the whole Hilbert space by applying $\hat{\psi}_{\ell}^{a \dagger}(\ell=k, k+1$ and $a=1,2)$ successively to the Fock vacuum $|0\rangle$ defined by $\hat{\psi}_{\ell}^{a}|0\rangle=0$ for all $\ell$ and $a=1,2$. Then, one reorganizes the Fock states according to the decomposition into representations.

Below, we explicitly show the basis of the Hilbert space according to the decomposition in Eq. (3.10). We introduce

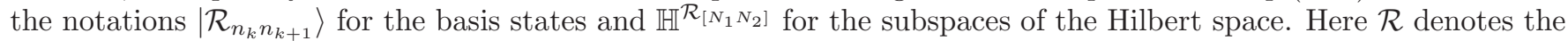
representation of pseudo-spin, and the subscript $n_{k} n_{k+1}\left(n_{k}, n_{k+1}=0,1,2\right)$ denotes the number of the Dirac fermions at the $k$-th and $(k+1)$-th vortices, respectively. In the subspaces, we do not distinguish $n_{k}$ and $n_{k+1}$ for the reasons mentioned later, and thus $N_{1}$ and $N_{2}$ are defined as $N_{1}=\max \left\{n_{k}, n_{k+1}\right\}$ and $N_{2}=\min \left\{n_{k}, n_{k+1}\right\}$. For example, $\left(N_{1}, N_{2}\right)=(1,0)$ contains two cases $\left(n_{k}, n_{k+1}\right)=(1,0),(0,1)$. Then, the whole Hilbert space of two Dirac vortices is decomposed into seven subspaces:

$$
\begin{aligned}
\mathbb{H}^{\{n=2\}} & =\mathbb{H}^{\mathbf{1}_{[00]}} \oplus \mathbb{H}^{\mathbf{1}_{[11]}} \oplus \mathbb{H}^{\mathbf{1}_{[20]}} \oplus \mathbb{H}^{\mathbf{1}_{[22]}} \\
& \oplus \mathbb{H}^{\mathbf{2}_{[10]}} \oplus \mathbb{H}^{\mathbf{2}_{[21]}} \\
& \oplus \mathbb{H}^{\mathbf{3}_{[11]}}
\end{aligned}
$$

This should be compared with the decomposition (3.10).

The first line in Eq. (3.11) corresponds to the singlet subspaces. They are defined by the basis states as $\mathbb{H}^{\mathbf{1}_{[00]}} \equiv$ $\left\{\left|\mathbf{1}_{00}\right\rangle\right\}, \mathbb{H}^{\mathbf{1}_{[11]}} \equiv\left\{\left|\mathbf{1}_{11}\right\rangle\right\}, \mathbb{H}^{\mathbf{1}_{[20]}} \equiv\left\{\left|\mathbf{1}_{20}\right\rangle,\left|\mathbf{1}_{02}\right\rangle\right\}$, and $\mathbb{H}^{\mathbf{1}_{[22]}} \equiv\left\{\left|\mathbf{1}_{22}\right\rangle\right\}$. Explicit forms of the basis states are given as follows:

$$
\begin{aligned}
\left|\mathbf{1}_{00}\right\rangle & \equiv|0\rangle \\
\left|\mathbf{1}_{11}\right\rangle & \equiv \frac{1}{\sqrt{2}}\left(\hat{\psi}_{k}^{1 \dagger} \hat{\psi}_{k+1}^{2 \dagger}-\hat{\psi}_{k}^{2 \dagger} \hat{\psi}_{k+1}^{1 \dagger}\right)|0\rangle \\
\left|\mathbf{1}_{20}\right\rangle & \equiv \hat{\psi}_{k}^{1 \dagger} \hat{\psi}_{k}^{2 \dagger}|0\rangle
\end{aligned}
$$




$$
\begin{aligned}
\left|\mathbf{1}_{02}\right\rangle & \equiv \hat{\psi}_{k+1}^{1 \dagger} \hat{\psi}_{k+1}^{2 \dagger}|0\rangle, \\
\left|\mathbf{1}_{22}\right\rangle & \equiv \hat{\psi}_{k}^{1 \dagger} \hat{\psi}_{k}^{2 \dagger} \hat{\psi}_{k+1}^{1 \dagger} \hat{\psi}_{k+1}^{2 \dagger}|0\rangle .
\end{aligned}
$$

The second line in Eq. (3.11) corresponds to the doublet subspaces. They are defined by the basis states as $\mathbb{H}^{\mathbf{2}^{[10]}} \equiv\left\{\left|\mathbf{2}_{10}\right\rangle,\left|\mathbf{2}_{01}\right\rangle\right\}$ and $\mathbb{H}^{\mathbf{2}_{[21]}} \equiv\left\{\left|\mathbf{2}_{21}\right\rangle,\left|\mathbf{2}_{12}\right\rangle\right\}$. Explicit forms of the basis states are given as follows:

$$
\begin{aligned}
\left|\mathbf{2}_{10}\right\rangle & \equiv\left(\begin{array}{c}
\hat{\psi}_{k}^{1 \dagger} \\
\hat{\psi}_{k}^{2 \dagger}
\end{array}\right)|0\rangle, \\
\left|\mathbf{2}_{01}\right\rangle & \equiv\left(\begin{array}{c}
\hat{\psi}_{k+1}^{1 \dagger} \\
\hat{\psi}_{k+1}^{2 \dagger}
\end{array}\right)|0\rangle, \\
\left|\mathbf{2}_{21}\right\rangle & \equiv \hat{\psi}_{k}^{1 \dagger} \hat{\psi}_{k}^{2 \dagger}\left(\begin{array}{c}
\hat{\psi}_{k+1}^{1 \dagger} \\
\hat{\psi}_{k+1}^{2 \dagger}
\end{array}\right)|0\rangle, \\
\left|\mathbf{2}_{12}\right\rangle & \equiv\left(\begin{array}{c}
\hat{\psi}_{k}^{1 \dagger} \\
\hat{\psi}_{k}^{2 \dagger}
\end{array}\right) \hat{\psi}_{k+1}^{1 \dagger} \hat{\psi}_{k+1}^{2 \dagger}|0\rangle .
\end{aligned}
$$

Here, in the last two expressions, we have factored out the singlet part so that the vector structure becomes manifest. Lastly, the third line in Eq. (3.11) corresponds to the triplet subspace. It is defined by the basis states as $\mathbb{H}^{\mathbf{3}^{[11]}} \equiv$ $\left\{\left|3_{11}\right\rangle\right\}$ with

$$
\left|\mathbf{3}_{11}\right\rangle \equiv\left(\begin{array}{c}
\hat{\psi}_{k}^{1 \dagger} \hat{\psi}_{k+1}^{1 \dagger} \\
\frac{1}{\sqrt{2}}\left(\hat{\psi}_{k}^{1 \dagger} \hat{\psi}_{k+1}^{2 \dagger}+\hat{\psi}_{k}^{2 \dagger} \hat{\psi}_{k+1}^{1 \dagger}\right) \\
\hat{\psi}_{k}^{2 \dagger} \hat{\psi}_{k+1}^{2 \dagger}
\end{array}\right)|0\rangle .
$$

Now we have prepared to discuss the exchange of vortices. We recall that the exchange of $\hat{\psi}_{k}^{a}$ and $\hat{\psi}_{k+1}^{a}$ is expressed by the operator $\hat{\tau}_{k}$ defined in Eq. (3.2) as a unitary transformation $\hat{\tau}_{k} \hat{\psi}_{\ell}^{a} \hat{\tau}_{k}^{-1}(\ell=k, k+1$ and $a=1,2)$. With the explicit forms of the basis states in Hilbert subspaces, we are able to express the operator $\hat{\tau}_{k}$ as matrices. In the

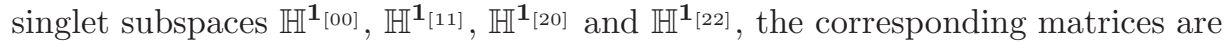

$$
\begin{aligned}
\tau_{k}^{\mathbf{1}_{[00]}} & =1, \\
\tau_{k}^{\mathbf{1}_{[11]}} & =-1, \\
\tau_{k}^{\mathbf{1}_{[20]}} & =\left(\begin{array}{ll}
0 & 1 \\
1 & 0
\end{array}\right), \\
\tau_{k}^{\mathbf{1}_{[22]}} & =1 .
\end{aligned}
$$

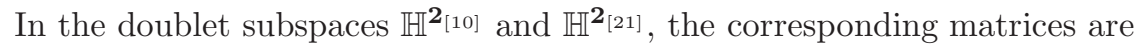

$$
\begin{aligned}
\tau_{k}^{\mathbf{2}_{[10]}} & =\left(\begin{array}{cc}
0 & -1 \\
1 & 0
\end{array}\right), \\
\tau_{k}^{\mathbf{2}_{[21]}} & =\left(\begin{array}{cc}
0 & 1 \\
-1 & 0
\end{array}\right) .
\end{aligned}
$$

Lastly, in the triplet subspace $\mathbb{H}^{\mathbf{3}^{[11]}}$, the matrix is

$$
\tau_{k}^{\mathbf{3}_{[11]}}=1
$$

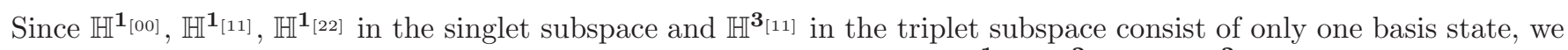

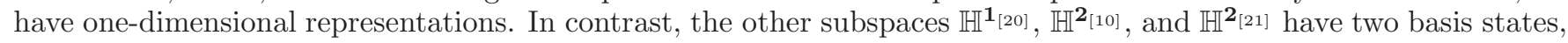
thus yielding two-dimensional representations. Notice that the exchange matrices in these subspaces have off-diagonal elements. However, this does not mean that the representation of the braid group is non-Abelian. Rather, this simply implies that two basis states are mixed with each other by the exchange operation (this is the reason why we did not specify the order of $n_{k}$ and $n_{k+1}$ in defining the subspaces). In fact, one can choose appropriate basis states so that the exchange matrices are expressed as diagonal. For example, $\tau_{k}^{\mathbf{1}^{[20]}}$ is diagonalized with eigenvalues \pm 1 showing the Abelian representation of the braid group, while $\tau_{k}^{\mathbf{2}_{[10]}}$ and $\tau_{k}^{\mathbf{2}_{[21]}}$ are diagonalized with $\pm i$, showing anyon-like Abelian representation of the braid group. 


\section{The case of $n=3$ : emergence of non-Abelian representation}

Let us finally consider the case of three Dirac vortices which are respectively labelled by $k, k+1$ and $k+2$. Basically we will follow the same procedures presented in the case of two Dirac vortices, but as easily expected, the analysis becomes quite involved. Still, we present here all the information since this is the simplest case where the non-Abelian representation of the braid group appears. In fact, when we have only two vortices $(k$-th and $(k+1)$-th vortices), there is only one exchange operator $\hat{\tau}_{k}$, thus we are not able to discuss the non-commutativity of two exchange operations. It makes sense only when we have three or more vortices. When we have three vortices $(k$-th, $(k+1)$-th and $(k+2)$-th vortices), we can define two exchange operators $\hat{\tau}_{k}$ and $\hat{\tau}_{k+1}$, and thus we can check if these two are commutative or not.

First of all, by using Eq. (3.8) for a single Dirac vortex, we can decompose the pseudo-spin structure made of three Dirac vortices into representations of $\mathrm{U}(2)$. We again introduce the notation $\mathcal{R}_{n_{k} n_{k+1} n_{k+2}}$ similar to the $n=2$ case. Here the subscript $n_{k} n_{k+1} n_{k+2}\left(n_{k}, n_{k+1}, n_{k+2}=0,1,2\right)$ denotes the number of Dirac fermions at the $k$-th, $(k+1)$-th and $(k+2)$-th vortices, respectively. Then, one finds

$$
\begin{aligned}
\left(\mathbf{1}_{0}\right. & \left.+\mathbf{2}_{1}+\mathbf{1}_{2}\right)_{\text {vortex } k} \otimes\left(\mathbf{1}_{0}+\mathbf{2}_{1}+\mathbf{1}_{2}\right)_{\text {vortex } k+1} \otimes\left(\mathbf{1}_{0}+\mathbf{2}_{1}+\mathbf{1}_{2}\right)_{\text {vortex } k+2} \\
& =\mathbf{1}_{000}+\left(\mathbf{1}_{020}+\mathbf{1}_{200}+\mathbf{1}_{002}\right)+\left(\mathbf{1}_{110}+\mathbf{1}_{011}+\mathbf{1}_{101}\right)+\left(\mathbf{1}_{220}+\mathbf{1}_{022}+\mathbf{1}_{202}\right)+\left(\mathbf{1}_{112}+\mathbf{1}_{211}+\mathbf{1}_{121}\right)+\mathbf{1}_{222} \\
& +\left(\mathbf{2}_{100}+\mathbf{2}_{010}+\mathbf{2}_{001}\right)+\left(\mathbf{2}_{210}+\mathbf{2}_{120}+\mathbf{2}_{012}+\mathbf{2}_{102}+\mathbf{2}_{021}+\mathbf{2}_{201}\right)+\left(\mathbf{2}_{212}+\mathbf{2}_{122}+\mathbf{2}_{221}\right)+\left(\mathbf{2}_{\mathrm{A} 111}+\mathbf{2}_{\mathrm{S} \underline{111}}\right) \\
& +\left(\mathbf{3}_{110}+\mathbf{3}_{011}+\mathbf{3}_{101}\right)+\left(\mathbf{3}_{112}+\mathbf{3}_{211}+\mathbf{3}_{121}\right) \\
& +\mathbf{4}_{\mathrm{S}} \underline{111} .
\end{aligned}
$$

Here we have also introduced new notations $\mathbf{2}_{\mathrm{A}} \underline{11}, \mathbf{2}_{\mathrm{S} 111}$ and $\mathbf{4}_{\mathrm{S}} \underline{111}$, meaning that the first two subscripts with underlines are made Asymmetric (Symmetric) with respect to the indices. We will note again when we present the explicit forms of the basis states. Each term corresponds to the basis state in the Hilbert subspace. Notice that we have already grouped the representations so as not to distinguish the ordering of $n_{k}, n_{k+1}$ and $n_{k+2}$. Thus, one can easily see that the whole Hilbert space $\mathbb{H}^{\{n=3\}}$ of three $\mathrm{U}(2)$ Dirac vortices can be decomposed into a direct sum

$$
\begin{aligned}
\mathbb{H}^{\{n=3\}} & =\mathbb{H}^{\mathbf{1}_{[000]}} \oplus \mathbb{H}^{\mathbf{1}_{[200]}} \oplus \mathbb{H}^{\mathbf{1}_{[110]}} \oplus \mathbb{H}^{\mathbf{1}_{[220]}} \oplus \mathbb{H}^{\mathbf{1}_{[211]}} \oplus \mathbb{H}^{\mathbf{1}_{[22]}} \\
& \oplus \mathbb{H}^{\mathbf{2}_{[100]}} \oplus \mathbb{H}^{\mathbf{2}_{[210]}} \oplus \mathbb{H}^{\mathbf{2}_{[221]}} \oplus \mathbb{H}^{\mathbf{2}_{[111]}} \\
& \oplus \mathbb{H}^{\mathbf{3}_{[110]}} \oplus \mathbb{H}^{\mathbf{3}_{[211]}} \\
& \oplus \mathbb{H}^{\mathbf{4}_{[111]}}
\end{aligned}
$$

Next, one can obtain the basis states of the Hilbert space by applying $\hat{\psi}_{\ell}^{a \dagger}(\ell=k, k+1, k+2$ and $a=1,2)$ successively to the vacuum $|0\rangle$ defined by $\hat{\psi}_{\ell}^{a}|0\rangle=0$ for all $\ell$ and $a=1,2$.

The first line in the decomposition (3.21) corresponds to the singlet subspaces. They are defined by the basis states as $\mathbb{H}^{\mathbf{1}_{[000]}} \equiv\left\{\left|\mathbf{1}_{000}\right\rangle\right\}, \mathbb{H}^{\mathbf{1}_{[200]}} \equiv\left\{\left|\mathbf{1}_{020}\right\rangle,\left|\mathbf{1}_{200}\right\rangle,\left|\mathbf{1}_{002}\right\rangle\right\}, \mathbb{H}^{\mathbf{1}_{[110]}} \equiv\left\{\left|\mathbf{1}_{110}\right\rangle,\left|\mathbf{1}_{011}\right\rangle,\left|\mathbf{1}_{101}\right\rangle\right\}, \mathbb{H}^{\mathbf{1}_{[220]}} \equiv\left\{\left|\mathbf{1}_{220}\right\rangle,\left|\mathbf{1}_{022}\right\rangle,\left|\mathbf{1}_{202}\right\rangle\right\}$, $\mathbb{H}^{\mathbf{1}_{[211]}} \equiv\left\{\left|\mathbf{1}_{112}\right\rangle,\left|\mathbf{1}_{211}\right\rangle,\left|\mathbf{1}_{121}\right\rangle\right\}$ and $\mathbb{H}^{\mathbf{1}_{[222]}} \equiv\left\{\left|\mathbf{1}_{222}\right\rangle\right\}$. Notice that all these states have even numbers of Dirac fermions. Explicit forms of the basis states are given as follows:

$$
\begin{aligned}
& \mathbb{H}^{\mathbf{1}_{[000]}:} \quad\left|\mathbf{1}_{000}\right\rangle \equiv|0\rangle, \\
& \mathbb{H}^{\mathbf{1}_{[200]}:} \quad\left\{\begin{aligned}
\left|\mathbf{1}_{020}\right\rangle & \equiv \hat{\psi}_{k+1}^{1 \dagger} \hat{\psi}_{k+1}^{2 \dagger}|0\rangle \\
\left|\mathbf{1}_{200}\right\rangle & \equiv \hat{\psi}_{k}^{1 \dagger} \hat{\psi}_{k}^{2 \dagger}|0\rangle \\
\left|\mathbf{1}_{002}\right\rangle & \equiv \hat{\psi}_{k+2}^{1 \dagger} \hat{\psi}_{k+2}^{2 \dagger}|0\rangle
\end{aligned}\right. \\
& \mathbb{H}^{\mathbf{1}_{[110]}:} \quad\left\{\begin{array}{l}
\left|\mathbf{1}_{110}\right\rangle \equiv \frac{1}{\sqrt{2}}\left(\hat{\psi}_{k}^{1 \dagger} \hat{\psi}_{k+1}^{2 \dagger}-\hat{\psi}_{k}^{2 \dagger} \hat{\psi}_{k+1}^{1 \dagger}\right)|0\rangle, \\
\left|\mathbf{1}_{011}\right\rangle \equiv \frac{1}{\sqrt{2}}\left(\hat{\psi}_{k+1}^{1 \dagger} \hat{\psi}_{k+2}^{2 \dagger}-\hat{\psi}_{k+1}^{2 \dagger} \hat{\psi}_{k+2}^{1 \dagger}\right)|0\rangle, \\
\left|\mathbf{1}_{101}\right\rangle \equiv \frac{1}{\sqrt{2}}\left(\hat{\psi}_{k}^{1 \dagger} \hat{\psi}_{k+2}^{2 \dagger}-\hat{\psi}_{k}^{2 \dagger} \hat{\psi}_{k+2}^{1 \dagger}\right)|0\rangle,
\end{array}\right. \\
& \mathbb{H}^{\mathbf{1}_{[220]}:} \quad\left\{\begin{aligned}
\left|\mathbf{1}_{220}\right\rangle & \equiv \hat{\psi}_{k}^{1 \dagger} \hat{\psi}_{k}^{2 \dagger} \hat{\psi}_{k+1}^{1 \dagger} \hat{\psi}_{k+1}^{2 \dagger}|0\rangle \\
\left|\mathbf{1}_{022}\right\rangle & \equiv \hat{\psi}_{k+1}^{1 \dagger} \hat{\psi}_{k+1}^{2 \dagger} \hat{\psi}_{k+2}^{1 \dagger} \hat{\psi}_{k+2}^{2 \dagger}|0\rangle \\
\left|\mathbf{1}_{202}\right\rangle & \equiv \hat{\psi}_{k}^{1 \dagger} \hat{\psi}_{k}^{2 \dagger} \hat{\psi}_{k+2}^{1 \dagger} \hat{\psi}_{k+2}^{2 \dagger}|0\rangle
\end{aligned}\right. \\
& \mathbb{H}^{\mathbf{1}_{[211]}:} \quad\left\{\begin{array}{l}
\left|\mathbf{1}_{112}\right\rangle \equiv \frac{1}{\sqrt{2}}\left(\hat{\psi}_{k}^{1 \dagger} \hat{\psi}_{k+1}^{2 \dagger}-\hat{\psi}_{k}^{2 \dagger} \hat{\psi}_{k+1}^{1 \dagger}\right) \hat{\psi}_{k+2}^{1 \dagger} \hat{\psi}_{k+2}^{2 \dagger}|0\rangle \\
\left|\mathbf{1}_{211}\right\rangle \equiv \frac{1}{\sqrt{2}} \hat{\psi}_{k}^{1 \dagger} \hat{\psi}_{k}^{2 \dagger}\left(\hat{\psi}_{k+1}^{1 \dagger} \hat{\psi}_{k+2}^{2 \dagger}-\hat{\psi}_{k+1}^{2 \dagger} \hat{\psi}_{k+2}^{1 \dagger}\right)|0\rangle \\
\left|\mathbf{1}_{121}\right\rangle \equiv \frac{1}{\sqrt{2}}\left(\hat{\psi}_{k}^{1 \dagger} \hat{\psi}_{k+2}^{2 \dagger}-\hat{\psi}_{k}^{2 \dagger} \hat{\psi}_{k+2}^{1 \dagger}\right) \hat{\psi}_{k+1}^{1 \dagger} \hat{\psi}_{k+1}^{2 \dagger}|0\rangle
\end{array}\right.
\end{aligned}
$$




$$
\mathbb{H}^{\mathbf{1}^{[222]}}: \quad \quad\left|\mathbf{1}_{222}\right\rangle \equiv \hat{\psi}_{k}^{1 \dagger} \hat{\psi}_{k}^{2 \dagger} \hat{\psi}_{k+1}^{1 \dagger} \hat{\psi}_{k+1}^{2 \dagger} \hat{\psi}_{k+2}^{1 \dagger} \hat{\psi}_{k+2}^{2 \dagger}|0\rangle .
$$

It is interesting to notice that the structures in $\mathbb{H}^{\mathbf{1}_{[110]}}$ and $\mathbb{H}^{\mathbf{1}_{[211]}}$, or in $\mathbb{H}^{\mathbf{1}_{[200]}}$ and $\mathbb{H}^{\mathbf{1}_{[220]}}$ are similar. This is the symmetry between "occupied" and "empty" states (or "particles" and "holes"), reflecting the ambiguity in defining the creation operator (i.e., we could define $\hat{\psi}_{k}^{a}$ as a creation operator, instead of the annihilation operator).

The second line of Eq. (3.21) corresponds to the doublet subspaces. These four subspaces are respectively defined by the basis states as $\mathbb{H}^{\left[\mathbf{2}^{[100]}\right.} \equiv\left\{\left|\mathbf{2}_{100}\right\rangle,\left|\mathbf{2}_{010}\right\rangle,\left|\mathbf{2}_{001}\right\rangle\right\}, \mathbb{H}^{\mathbf{2}_{[210]}} \equiv\left\{\left|\mathbf{2}_{210}\right\rangle,\left|\mathbf{2}_{120}\right\rangle,\left|\mathbf{2}_{012}\right\rangle,\left|\mathbf{2}_{102}\right\rangle,\left|\mathbf{2}_{021}\right\rangle,\left|\mathbf{2}_{201}\right\rangle\right\}, \mathbb{H}^{\mathbf{2}_{[221]}} \equiv$ $\left\{\left|\mathbf{2}_{212}\right\rangle,\left|\mathbf{2}_{122}\right\rangle,\left|\mathbf{2}_{221}\right\rangle\right\}$, and $\mathbb{H}^{\mathbf{2}_{[11]}} \equiv\left\{\left|\mathbf{2}_{\mathrm{A}} \underline{111}\right\rangle,\left|\mathbf{2}_{\mathrm{S}} \underline{111}\right\rangle\right\}$. This time, the total numbers of Dirac fermions are odd in these states. Explicit forms of the basis states are given as follows:

$$
\begin{aligned}
& \mathbb{H}^{\mathbf{2}_{[100]}}: \quad\left\{\begin{aligned}
\left|\mathbf{2}_{100}\right\rangle & \equiv\left(\begin{array}{c}
\hat{\psi}_{k}^{1 \dagger} \\
\hat{\psi}_{k}^{2 \dagger}
\end{array}\right)|0\rangle \\
\left|\mathbf{2}_{010}\right\rangle & \equiv\left(\begin{array}{c}
\hat{\psi}_{k+1}^{1 \dagger} \\
\hat{\psi}_{k+1}^{2 \dagger}
\end{array}\right)|0\rangle \\
\left|\mathbf{2}_{001}\right\rangle & \equiv\left(\begin{array}{c}
\hat{\psi}_{k+2}^{1 \dagger} \\
\hat{\psi}_{k+2}^{2 \dagger}
\end{array}\right)|0\rangle
\end{aligned}\right. \\
& \left\{\begin{aligned}
&\left|\mathbf{2}_{210}\right\rangle \equiv \hat{\psi}_{k}^{1 \dagger} \hat{\psi}_{k}^{2 \dagger}\left(\begin{array}{c}
\hat{\psi}_{k+1}^{1 \dagger} \\
\hat{\psi}_{k+1}^{2 \dagger}
\end{array}\right)|0\rangle, \\
&\left|\mathbf{2}_{120}\right\rangle \equiv\left(\begin{array}{c}
\hat{\psi}_{k}^{1 \dagger} \\
\hat{\psi}_{k}^{2 \dagger}
\end{array}\right) \hat{\psi}_{k+1}^{1 \dagger} \hat{\psi}_{k+1}^{2 \dagger}|0\rangle, \\
&\left.\mathbf{2}_{0120]}\right\rangle \equiv\left(\begin{array}{c}
\hat{\psi}_{k+1}^{1 \dagger} \\
\hat{\psi}_{k+1}^{2 \dagger}
\end{array}\right) \hat{\psi}_{k+2}^{1 \dagger} \hat{\psi}_{k+2}^{2 \dagger}|0\rangle, \\
&\left|\mathbf{2}_{102}\right\rangle \equiv\left(\begin{array}{c}
\hat{\psi}_{k}^{1 \dagger} \\
\hat{\psi}_{k}^{1 \dagger}
\end{array}\right) \hat{\psi}_{k+2}^{1 \dagger} \hat{\psi}_{k+2}^{2 \dagger}|0\rangle, \\
&\left|\mathbf{2}_{021}\right\rangle \equiv \hat{\psi}_{k+1}^{1 \dagger} \hat{\psi}_{k+1}^{2 \dagger}\left(\begin{array}{c}
\hat{\psi}_{k+2}^{1 \dagger} \\
\hat{\psi}_{k+2}^{2 \dagger}
\end{array}\right)|0\rangle, \\
&\left|\mathbf{2}_{201}\right\rangle \equiv \hat{\psi}_{k}^{1 \dagger} \hat{\psi}_{k}^{2 \dagger}\left(\begin{array}{c}
\hat{\psi}_{k+2}^{1 \dagger} \\
\hat{\psi}_{k+2}^{2 \dagger}
\end{array}\right)|0\rangle,
\end{aligned}\right. \\
& \mathbb{H}^{2_{[221]}}: \quad\left\{\begin{array}{l}
\left|\mathbf{2}_{212}\right\rangle \equiv \hat{\psi}_{k}^{1 \dagger} \hat{\psi}_{k}^{2 \dagger}\left(\begin{array}{c}
\hat{\psi}_{k+1}^{1 \dagger} \\
\hat{\psi}_{k+1}^{2 \dagger}
\end{array}\right) \hat{\psi}_{k+2}^{1 \dagger} \hat{\psi}_{k+2}^{2 \dagger}|0\rangle, \\
\left|\mathbf{2}_{122}\right\rangle \equiv\left(\begin{array}{c}
\hat{\psi}_{k}^{1 \dagger} \\
\hat{\psi}_{k}^{2 \dagger}
\end{array}\right) \hat{\psi}_{k+1}^{1 \dagger} \hat{\psi}_{k+1}^{2 \dagger} \hat{\psi}_{k+2}^{1 \dagger} \hat{\psi}_{k+2}^{2 \dagger}|0\rangle, \\
\left|\mathbf{2}_{221}\right\rangle \equiv \hat{\psi}_{k}^{1 \dagger} \hat{\psi}_{k}^{2 \dagger} \hat{\psi}_{k+1}^{1 \dagger} \hat{\psi}_{k+1}^{2 \dagger}\left(\begin{array}{c}
\hat{\psi}_{k+2}^{1 \dagger} \\
\hat{\psi}_{k+2}^{2 \dagger}
\end{array}\right)|0\rangle,
\end{array}\right. \\
& \mathbb{H}^{\mathbf{2}^{[111]}}: \quad\left\{\begin{array}{l}
\left|\mathbf{2}_{\mathrm{A}} \underline{111}\right\rangle \equiv \frac{1}{\sqrt{2}}\left(\hat{\psi}_{k}^{1 \dagger} \hat{\psi}_{k+1}^{2 \dagger}-\hat{\psi}_{k}^{2 \dagger} \hat{\psi}_{k+1}^{1 \dagger}\right)\left(\begin{array}{c}
\hat{\psi}_{k+2}^{1 \dagger} \\
\hat{\psi}_{k+2}^{2 \dagger}
\end{array}\right)|0\rangle, \\
\left|\mathbf{2}_{\mathrm{S}} \underline{111}\right\rangle \equiv \frac{1}{\sqrt{6}}\left(\begin{array}{c}
2 \hat{\psi}_{k}^{1 \dagger} \hat{\psi}_{k+1}^{1 \dagger} \hat{\psi}_{k+2}^{2 \dagger}-\left(\hat{\psi}_{k}^{1 \dagger} \hat{\psi}_{k+1}^{2 \dagger}+\hat{\psi}_{k}^{2 \dagger} \hat{\psi}_{k+1}^{1 \dagger}\right) \hat{\psi}_{k+2}^{1 \dagger} \\
\left(\hat{\psi}_{k}^{1 \dagger} \hat{\psi}_{k+1}^{2 \dagger}+\hat{\psi}_{k}^{2 \dagger} \hat{\psi}_{k+1}^{1 \dagger}\right) \hat{\psi}_{k+2}^{2 \dagger}-2 \hat{\psi}_{k}^{2 \dagger} \hat{\psi}_{k+1}^{2 \dagger} \hat{\psi}_{k+2}^{1 \dagger}
\end{array}\right)|0\rangle .
\end{array}\right.
\end{aligned}
$$

Now the meaning of the notations $\mathrm{A}$ and $\mathrm{S}$ is evident. For example, in $\left|\mathbf{2}_{\mathrm{A}} \underline{111}\right\rangle$ the first $(k$-th) and second $((k+1)$-th $)$ vortices form anti-symmetric combination with respect to the indices.

The third line of the decomposition (3.21) corresponds to the triplet subspaces. They are defined by the basis states as $\mathbb{H}^{\mathbf{3}_{[110]}} \equiv\left\{\left|\mathbf{3}_{110}\right\rangle,\left|\mathbf{3}_{011}\right\rangle,\left|\mathbf{3}_{101}\right\rangle\right\}$ and $\mathbb{H}^{\mathbf{3}_{[211]}} \equiv\left\{\left|\mathbf{3}_{112}\right\rangle,\left|\mathbf{3}_{211}\right\rangle,\left|\mathbf{3}_{121}\right\rangle\right\}$. Total fermion numbers are now even. Explicit 
forms of the basis states are given as follows:

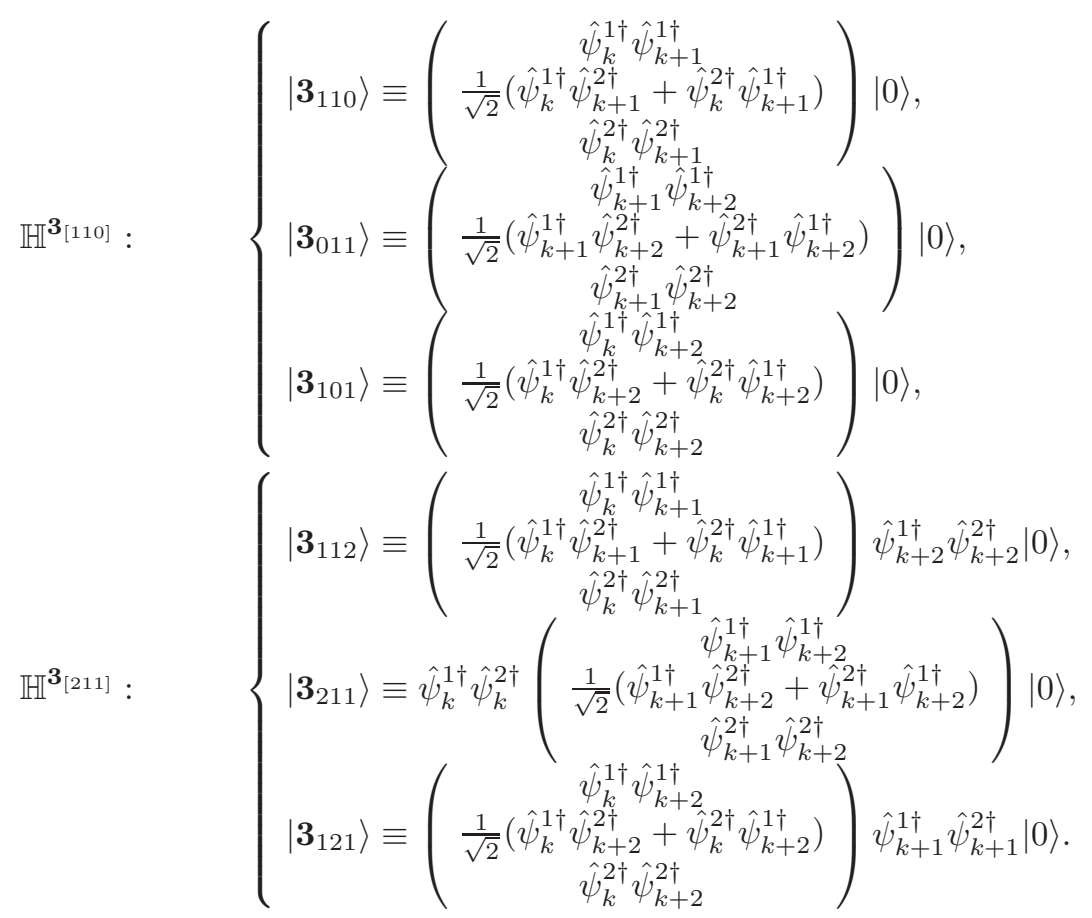

The last line of the decomposition 3.21) corresponds to the quartet subspace. It is defined by the basis states as $\mathbb{H}^{\mathbf{4}_{[111]}} \equiv\left\{\left|\mathbf{4}_{\mathrm{S}} \underline{111}\right\rangle\right\}$. Explicitly,

$$
\mathbb{H}^{\mathbf{4}_{[111]}:} \quad\left|\mathbf{4}_{\mathrm{S}} \underline{111}\right\rangle \equiv\left(\begin{array}{c}
\hat{\psi}_{k}^{1 \dagger} \hat{\psi}_{k+1}^{1 \dagger} \hat{\psi}_{k+2}^{1 \dagger} \\
\frac{1}{\sqrt{3}} \hat{\psi}_{k}^{1 \dagger} \hat{\psi}_{k+1}^{1 \dagger} \hat{\psi}_{k+2}^{2 \dagger}+\frac{1}{\sqrt{3}}\left(\hat{\psi}_{k}^{1 \dagger} \hat{\psi}_{k+1}^{2 \dagger}+\hat{\psi}_{k}^{2 \dagger} \hat{\psi}_{k+1}^{1 \dagger}\right) \hat{\psi}_{k+2}^{1 \dagger} \\
\frac{1}{\sqrt{3}}\left(\hat{\psi}_{k}^{1 \dagger} \hat{\psi}_{k+1}^{2 \dagger}+\hat{\psi}_{k}^{2 \dagger} \hat{\psi}_{k+1}^{1 \dagger}\right) \hat{\psi}_{k+2}^{2 \dagger}+\frac{1}{\sqrt{3}} \hat{\psi}_{k}^{2 \dagger} \hat{\psi}_{k+1}^{2 \dagger} \hat{\psi}_{k+2}^{1 \dagger} \hat{\psi}_{k}^{2 \dagger} \hat{\psi}_{k+1}^{2 \dagger} \hat{\psi}_{k+2}^{2 \dagger}
\end{array}\right)|0\rangle .
$$

Now we can finally discuss the exchange of $\mathrm{U}(2)$ Dirac vortices in the Hilbert space constructed for three Dirac vortices. As in the case of $n=2$, we express the operators $\hat{\tau}_{k}$ and $\hat{\tau}_{k+1}$ as matrices with the basis states presented above.

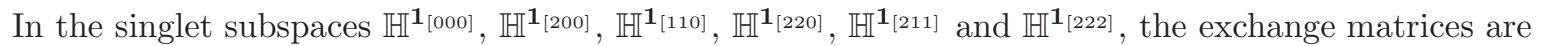

$$
\begin{aligned}
\tau_{k}^{\mathbf{1}_{[000]}} & =\tau_{k+1}^{\mathbf{1}_{[000]}}=1, \\
\tau_{k}^{\mathbf{1}_{[200]}} & =\left(\begin{array}{lll}
0 & 1 & 0 \\
1 & 0 & 0 \\
0 & 0 & 1
\end{array}\right), \quad \tau_{k+1}^{\mathbf{1}_{[200]}}=\left(\begin{array}{lll}
0 & 0 & 1 \\
0 & 1 & 0 \\
1 & 0 & 0
\end{array}\right) \\
\tau_{k}^{\mathbf{1}_{[110]}} & =\left(\begin{array}{ccc}
-1 & 0 & 0 \\
0 & 0 & 1 \\
0 & -1 & 0
\end{array}\right), \quad \tau_{k+1}^{\mathbf{1}_{[110]}}=\left(\begin{array}{ccc}
0 & 0 & -1 \\
0 & -1 & 0 \\
1 & 0 & 0
\end{array}\right), \\
\tau_{k}^{\mathbf{1}_{[220]}} & =\left(\begin{array}{ccc}
1 & 0 & 0 \\
0 & 0 & 1 \\
0 & 1 & 0
\end{array}\right), \quad \tau_{k+1}^{\mathbf{1}_{[220]}}=\left(\begin{array}{ccc}
0 & 0 & 1 \\
0 & 1 & 0 \\
1 & 0 & 0
\end{array}\right), \\
\tau_{k}^{\mathbf{1}_{[211]}} & =\left(\begin{array}{ccc}
-1 & 0 & 0 \\
0 & 0 & 1 \\
0 & -1 & 0
\end{array}\right), \quad \tau_{k+1}^{\mathbf{1}_{[211]}}=\left(\begin{array}{ccc}
0 & 0 & -1 \\
0 & -1 & 0 \\
1 & 0 & 0
\end{array}\right), \\
\tau_{k}^{\mathbf{1}_{[222]}} & =\tau_{k+1}^{\mathbf{1}_{[22]}}=1 .
\end{aligned}
$$

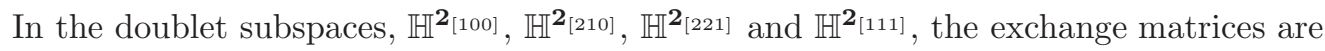

$$
\tau_{k}^{\mathbf{2}_{[100]}}=\left(\begin{array}{ccc}
0 & -1 & 0 \\
1 & 0 & 0 \\
0 & 0 & 1
\end{array}\right), \quad \tau_{k+1}^{\mathbf{2}_{[100]}}=\left(\begin{array}{ccc}
1 & 0 & 0 \\
0 & 0 & -1 \\
0 & 1 & 0
\end{array}\right),
$$




$$
\begin{aligned}
\tau_{k}^{\mathbf{2}_{[210]}} & =\left(\begin{array}{cccccc}
0 & 1 & 0 & 0 & 0 & 0 \\
-1 & 0 & 0 & 0 & 0 & 0 \\
0 & 0 & 0 & 1 & 0 & 0 \\
0 & 0 & -1 & 0 & 0 & 0 \\
0 & 0 & 0 & 0 & 0 & 1 \\
0 & 0 & 0 & 0 & 1 & 0
\end{array}\right), \quad \tau_{k+1}^{\mathbf{2}_{[210]}}=\left(\begin{array}{cccccc}
0 & 0 & 0 & 0 & 0 & -1 \\
0 & 0 & 0 & 1 & 0 & 0 \\
0 & 0 & 0 & 0 & -1 & 0 \\
0 & 1 & 0 & 0 & 0 & 0 \\
0 & 0 & 1 & 0 & 0 & 0 \\
1 & 0 & 0 & 0 & 0 & 0
\end{array}\right), \\
\tau_{k}^{\mathbf{2}_{[221]}} & =\left(\begin{array}{ccc}
0 & 1 & 0 \\
-1 & 0 & 0 \\
0 & 0 & 1
\end{array}\right), \quad \tau_{k+1}^{\mathbf{2}_{[221]}}=\left(\begin{array}{ccc}
0 & 0 & -1 \\
0 & 1 & 0 \\
1 & 0 & 0
\end{array}\right), \\
\tau_{k}^{\mathbf{2}_{[111]}} & =\left(\begin{array}{cc}
-1 & 0 \\
0 & 1
\end{array}\right), \quad \tau_{k+1}^{\mathbf{2}_{[111]}}=\left(\begin{array}{cc}
\frac{1}{2} & \frac{\sqrt{3}}{2} \\
\frac{\sqrt{3}}{2} & -\frac{1}{2}
\end{array}\right) .
\end{aligned}
$$

In the triplet subspaces, $\mathbb{H}^{\mathbf{3}_{[110]}}$ and $\mathbb{H}^{\mathbf{3}_{[211]}}$, the exchange matrices are

$$
\begin{aligned}
\tau_{k}^{\mathbf{3}_{[110]}}=\left(\begin{array}{ccc}
1 & 0 & 0 \\
0 & 0 & 1 \\
0 & -1 & 0
\end{array}\right), & \tau_{k+1}^{\mathbf{3}_{[110]}}=\left(\begin{array}{ccc}
0 & 0 & -1 \\
0 & 1 & 0 \\
1 & 0 & 0
\end{array}\right), \\
\tau_{k}^{\mathbf{3}_{[211]}}=\left(\begin{array}{ccc}
1 & 0 & 0 \\
0 & 0 & 1 \\
0 & -1 & 0
\end{array}\right), & \tau_{k+1}^{\mathbf{3}_{[211]}}=\left(\begin{array}{ccc}
0 & 0 & -1 \\
0 & 1 & 0 \\
1 & 0 & 0
\end{array}\right) .
\end{aligned}
$$

Lastly, in the quartet subspace $\mathbb{H}^{\mathbf{4}_{[111]}}$, the exchange matrices are

$$
\tau_{k}^{\mathbf{4}_{[111]}}=\tau_{k+1}^{\mathbf{4}_{[111]}}=1
$$

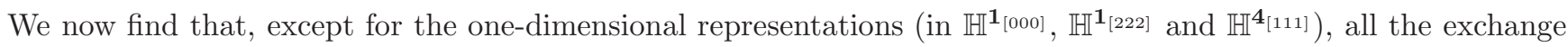
matrices are non-commutative; $\left[\tau_{k}, \tau_{k+1}\right] \neq 0$. Therefore, the exchange operations in these subspaces exhibit nonAbelian representation of the braid group.

Recall that we have shown that the exchange operators $\hat{\tau}_{\ell}$ 's satisfy $\left(\hat{\tau}_{\ell}\right)^{4}=1$ at the operator level (see Eq. (3.3)). Of course this is satisfied by all the exchange matrices obtained above. However, interestingly, a stronger relation

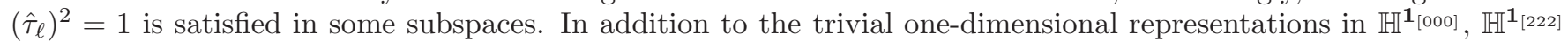

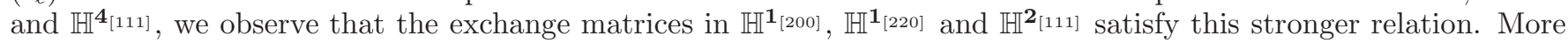
details are discussed in Appendix C.

We can continue the construction of the Hilbert space when the number of vortices is more than three. The dimension of the total Hilbert space of $n$-vortex system is $\left(2^{2}\right)^{n}$. We present the results for $n=4$ vortices in Appendix B] which also show non-Abelian representation of the braid group.

\section{U(1) DIRAC STRUCTURE EMBEDDED IN U(2) DIRAC VORTICES}

Recall that we already found in the previous paper [1] that the system of U(1) Dirac vortices shows non-Abelian representation of the braid group. Then, it is natural to ask how the previous results are related to the present results. In this section, we discuss that we can indeed identify the same structure as the U(1) vortices by a simple reduction of the $\mathrm{U}(2)$ system.

So far, we have not specified any detail of the interaction, but suppose that one can turn on an interaction which breaks the global $\mathrm{U}(2)$ symmetry so that only the $\mathrm{U}(1)$ symmetry is preserved. Then, in the presence of such an interaction, only one Dirac fermion would remain massless. We can realize such a case by simply ignoring the lower component $\hat{\psi}_{\ell}^{2}(\ell=1, \cdots, n)$ of the $\mathrm{U}(2)$ Dirac fermions. Under this simple procedure, the Hilbert space $\mathbb{H}^{\{n\}}$ of the $\mathrm{U}(2)$ Dirac vortices reduces to the Hilbert space $\mathbb{H}^{(n)}$ of the U(1) Dirac vortices discussed in the previous paper 11 ] (and also in Sec. II and Appendix A). Let us discuss the cases of $n=2$ and 3, separately.

Consider first the case of $n=2$ (see Sec. IIIB). One finds that only the following states survive after the reduction:

$$
\left|\mathbf{1}_{00}\right\rangle=|0\rangle, \quad\left|\mathbf{2}_{10}\right\rangle=\left(\begin{array}{c}
\hat{\psi}_{k}^{1 \dagger} \\
0
\end{array}\right)|0\rangle, \quad\left|\mathbf{2}_{01}\right\rangle=\left(\begin{array}{c}
\hat{\psi}_{k+1}^{1 \dagger} \\
0
\end{array}\right)|0\rangle, \quad\left|\mathbf{3}_{11}\right\rangle=\left(\begin{array}{c}
\hat{\psi}_{k}^{1 \dagger} \hat{\psi}_{k+1}^{1 \dagger} \\
0 \\
0
\end{array}\right)|0\rangle .
$$




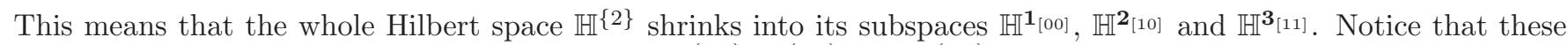
four states are equivalent to the basis states for $\mathbb{H}^{(2,0)}, \mathbb{H}^{(2,1)}$ and $\mathbb{H}^{(2,2)}$ in the U(1) Dirac vortices. Thus, it is not surprising that the exchange matrices in the reduced U(2) Dirac vortices are equivalent to those of the U(1) vortices:

$$
\begin{aligned}
\tau_{k}^{\mathbf{1}_{[00]}} & =\tau_{k}^{(2,0)}, \\
\tau_{k}^{\mathbf{2}_{[10]}} & =\tau_{k}^{(2,1)}, \\
\tau_{k}^{\mathbf{3}_{[11]}} & =\tau_{k}^{(2,2)} .
\end{aligned}
$$

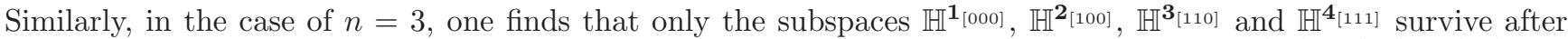
the reduction of the lower component. These Hilbert subspaces correspond, respectively, to $\mathbb{H}^{(3,0)}, \mathbb{H}^{(3,1)}, \mathbb{H}^{(3,2)}$ and $\mathbb{H}^{(3,3)}$ in the $\mathrm{U}(1)$ Dirac vortices. The matrices of these two cases are equivalent to each other:

$$
\begin{aligned}
\tau_{\ell}^{\mathbf{1}_{[000]}} & =\tau_{\ell}^{(3,0)}, \\
\tau_{\ell}^{\mathbf{2}_{[100]}} & =\tau_{\ell}^{(3,1)}, \\
\tau_{\ell}^{\mathbf{3}_{[110]}} & =\tau_{\ell}^{(3,2)}, \\
\tau_{\ell}^{\mathbf{4}_{[111]}} & =\tau_{\ell}^{(3,3)},
\end{aligned}
$$

with $\ell=k, k+1$.

By repeating the same arguments for general $n$, one can easily find that there will be a correspondence between the Hilbert subspaces $\mathbb{H}^{\mathcal{M}^{[1 \cdots 10 \cdots 0]}}$ and $\mathbb{H}^{(n, f)}$. The matrices in these two subspaces will be equivalent;

$$
\tau_{\ell}^{\mathcal{M}_{[1 \cdots 10 \cdots 0]}}=\tau_{\ell}^{(n, f)}
$$

with $\ell=1, \cdots, n-1$. Here, the $\mathrm{U}(2)$ representation $\mathcal{M}_{[\underbrace{1 \cdots 1}_{f}}^{10 \cdots 0} \underbrace{0 \cdots}_{n-f}$ contains $f$ Dirac fermions, and the dimension of $\mathcal{M}_{[1 \cdots 10 \cdots 0]}$ is equal to $f+1$. Thus, the Hilbert space $\mathbb{H}^{\{n\}}$ of the $\mathrm{U}(2)$ Dirac vortices covers $\mathbb{H}^{(n)}$ of the $\mathrm{U}(1)$ Dirac vortices. This is confirmed also for $n=4$ vortices in Appendix B.

\section{GENUINE NON-ABELIAN STATISTICS}

\section{A. Sectors of exchanges of the identical vortices}

We have constructed the braid group made of exchanges of $U(2)$ Dirac vortices. One may be able to regard it as a kind of exchange statistics since all the Dirac vortices to be exchanged are energetically the same. However, this is true only if we are allowed to neglect the difference between the occupation and absence of Dirac fermions in each vortex. Genuine non-Abelian statistics in a strict sense should appear in exchanges of identical particles, which implies that we should distinguish the number of Dirac fermions in each vortex. In the case of vortices with U(1) Dirac fermions, the whole braid group representation is non-Abelian, but does not have genuine non-Abelian statistics when restricted to a subspace of exchanges of identical states (i.e., between two occupied states or two un-occupied states). What is truly remarkable in the $\mathrm{U}(2)$ case is that the braid group which we have found indeed contains genuine non-Abelian statistics as its subgroup where we consider only the exchanges between the identical vortices, the vortices with the same numbers of Dirac fermions. Below, we explicitly show them in subspaces with $n=3$ and 4 vortices.

1. The doublet Hilbert subspace $\mathbb{H}^{\mathbf{2}_{[111]}}$ for three $\mathrm{U}(2)$ Dirac vortices $(n=3 ; k$-th, $(k+1)$-th and $(k+2)$-th vortices) with single Dirac fermion occupations. The minimum genuine non-Abelian statistics appears in the doublet Hilbert subspace $\mathbb{H}^{\mathbf{2}_{[111]}}$ in $n=3$ vortices. The two-dimensional Hilbert subspace $\mathbb{H}^{\mathbf{2}_{[111]}}$ is spanned by the basis $\left|\mathbf{2}_{\mathrm{A}} \underline{11} 1\right\rangle$ and $\left|\mathbf{2}_{\mathrm{S}} \underline{111}\right\rangle$ defined in Eq. (3.31). The Dirac fermion numbers in each U(2) Dirac vortex are one so that they are all identical vortices. Two exchange matrices, i.e., $\tau_{k}^{\mathbf{2}_{[11]]}}$ for the exchange of the $k$-th and $(k+1)$-th vortices, and $\tau_{k+1}^{\mathbf{2}_{[11]}}$ for the exchange of the $(k+1)$-th and $(k+2)$-th vortices, can be found in the last line in Eq. (3.35):

$$
\tau_{k}^{\mathbf{2}_{[111]}}=\left(\begin{array}{cc}
-1 & 0 \\
0 & 1
\end{array}\right), \quad \tau_{k+1}^{\mathbf{2}_{[111]}}=\left(\begin{array}{cc}
\frac{1}{2} & \frac{\sqrt{3}}{2} \\
\frac{\sqrt{3}}{2} & -\frac{1}{2}
\end{array}\right)
$$

They are non-commutative: $\tau_{k}^{\mathbf{2}_{[111]}} \tau_{k+1}^{\mathbf{2}_{[11]}} \neq \tau_{k+1}^{\mathbf{2}_{[111]}} \tau_{k}^{\mathbf{2}_{[111]}}$, showing genuine non-Abelian statistics. 
2. The non-Abelian transformation equivalent to Eq. (5.10) are embedded in a sector of four vortices. For instance, let us consider four vortices three of which contain one Dirac fermion at each vortex but the rest of which contains no Dirac fermion, then the exchange of the identical vortices with the one Dirac fermion occupation leads to the same result as in Eq. (5.10). To see this more explicitly, consider the doublet subspace with the basis $\left|\mathbf{2}_{\mathrm{A}} 01 \underline{11}\right\rangle$ and $\left|\mathbf{2}_{\mathrm{S} 0111}\right\rangle$ defined in Eq. (B.13). We can see that the submatrices made of the fifth and sixth columns and rows in $\tau_{k+1}^{\mathbf{2}_{[1110]}}$ and $\tau_{k+2}^{\mathbf{2}_{[1110]}}$ in Eq. (B.36) are nothing but the matrices in Eq. (5.10). The rest corresponding submatrix in $\tau_{k}^{\mathbf{2}_{[1110]}}$ in Eq. B.36) is consistently zero, because we are considering the exchange of only three identical vortices with one Dirac fermion occupations.

3. The singlet Hilbert space $\mathbb{H}^{\mathbf{1}_{[1111]}}$ for four $\mathrm{U}(2)$ Dirac vortices $(n=4 ; k$-th, $(k+1)$-th, $(k+2)$-th and $(k+3)$-th vortices) with single Dirac fermion occupations. We have now four identical vortices each of which contains one Dirac fermion. The singlet Hilbert space $\mathbb{H}^{\mathbf{1}_{[111]}}$ is a two-dimensional subspace with the basis $\left|\mathbf{1}_{\mathrm{AA}} \underline{11} \underline{11}\right\rangle$ and $\left|\mathbf{1}_{\mathrm{SS} \text { 11 } 11}\right\rangle$ defined in Eq. (B.8). The non-Abelian exchange matrices can be found in Eq. (B.31) as

$$
\tau_{k}^{\mathbf{1}_{[1111]}}=\left(\begin{array}{cc}
-1 & 0 \\
0 & 1
\end{array}\right), \tau_{k+1}^{\mathbf{1}_{[1111]}}=\left(\begin{array}{cc}
\frac{1}{2} & \frac{\sqrt{3}}{2} \\
\frac{\sqrt{3}}{2} & -\frac{1}{2}
\end{array}\right), \tau_{k+2}^{\mathbf{1}_{[1111]}}=\left(\begin{array}{cc}
-1 & 0 \\
0 & 1
\end{array}\right)
$$

The exchange of the Dirac vortices are non-Abelian; $\tau_{\ell}^{\mathbf{1}_{[1111]}} \tau_{\ell+1}^{\mathbf{1}_{[1111]}} \neq \tau_{\ell+1}^{\mathbf{1}_{[111]}} \tau_{\ell}^{\mathbf{1}_{[1111]}}$ for $\ell=k, k+1$.

4. The triplet Hilbert space $\mathbb{H}^{\mathbf{3}_{[111]}}$ for four $\mathrm{U}(2)$ Dirac vortices $(n=4 ; k$-th, $(k+1)$-th, $(k+2)$-th and $(k+3)$-th vortices) with single Dirac fermion occupations. The four identical Dirac vortices with single Dirac fermion occupations allow for another sector having genuine non-Abelian statistics. The triplet Hilbert space $\mathbb{H}^{\mathbf{3}_{[1111]}}$ is three dimensional with the basis $\left|\mathbf{3}_{\mathrm{AS}} \underline{11} \underline{11}\right\rangle,\left|\mathbf{3}_{\mathrm{SA}} \underline{11} \underline{11}\right\rangle$ and $\left|\mathbf{3}_{\mathrm{SS}} \underline{11} \underline{11}\right\rangle$ in Eq. (B.19). The exchange matrices can be found in Eq. (B.42) as

$$
\tau_{k}^{\mathbf{3}_{[1111]}}=\left(\begin{array}{ccc}
-1 & 0 & 0 \\
0 & 1 & 0 \\
0 & 0 & 1
\end{array}\right), \tau_{k+1}^{\mathbf{3}_{[111]}}=\left(\begin{array}{ccc}
\frac{1}{2} & -\frac{1}{2} & \frac{1}{\sqrt{2}} \\
-\frac{1}{2} & \frac{1}{2} & \frac{1}{\sqrt{2}} \\
\frac{1}{\sqrt{2}} & \frac{1}{\sqrt{2}} & 0
\end{array}\right), \tau_{k+2}^{\mathbf{3}_{[1111]}}=\left(\begin{array}{ccc}
1 & 0 & 0 \\
0 & -1 & 0 \\
0 & 0 & 1
\end{array}\right)
$$

The exchange of these Dirac vortices are non-Abelian; $\tau_{\ell}^{\mathbf{3}_{[1111]}} \tau_{\ell+1}^{\mathbf{3}_{[1111]}} \neq \tau_{\ell+1}^{\mathbf{3}_{[1111]}} \tau_{\ell}^{\mathbf{3}_{[1111]}}$ for $\ell=k, k+1$.

From the above examples, we find that the U(2) Dirac vortices with one Dirac fermion occupation give non-Abelian quantum statistics of identical particles.

\section{B. Entanglement in the Hilbert space}

If one reminds of the case of Majorana vortices showing the genuine non-Abelian statistics, one may critically wonder why we have found the genuine non-Abelian statistics even though Dirac fermions are defined locally at each vortex. As is well known, for vortices with Majorana fermions, Dirac fermions have to be defined non-locally by using two Majorana fermions localized at spatially separated vortices, and, thus far, it has been commonly thought that emergence of non-Abelian statistics is attributed to such a non-locality of Dirac fermions. In contrast, in our case, Dirac fermions are introduced from the beginning, and are of course defined locally at each vortex. Then, it is natural to ask why we get non-Abelian statistics from local Dirac fermions, or to raise question if the non-locality is really essential for the emergence of non-Abelian statistics.

Our answer is that the non-locality is indeed essential but it does not come from the definition of Dirac fermions but rather from the definition of the basis of the Hilbert space. Namely, the use of entangled states, which was absent in the previous $\mathrm{U}(1)$ Dirac case, is essential in the $\mathrm{U}(2)$ case. For instance, in the first example of the previous subsection, the basis $\left|\mathbf{2}_{\mathrm{A} 111}\right\rangle$ and $\left|\mathbf{2}_{\mathrm{S} 111}\right\rangle$ in Eq. (3.31) are entangled because of (anti-)symmetric combination of indices. Such entangled states are necessary because the basis of the Hilbert space have to belong to the irreducible representations of $\mathrm{U}(2)$ symmetry. Therefore, genuine non-Abelian statistics does not appear in U(1) Dirac vortices but it first appears in U(2) Dirac vortices because of non-Abelian U(2) symmetry group acting on the doublet Dirac fermions on the vortices.

From these considerations, we conclude that some kind of non-locality is necessary to obtain the genuine nonAbelian statistics: It is the non-local definition of the Dirac fermions in the case of Majorana vortices, while it is the entanglement in the Hilbert space in the case of Dirac vortices. 


\section{SUMMARY}

We have considered the simplest system of non-Abelian Dirac vortices, namely the system of vortices in which U(2) doublet zero-energy Dirac fermions are trapped. We have constructed the non-Abelian representation of the braid group for the exchange of vortices with $\mathrm{U}(2)$ Dirac fermions. This was confirmed both at the operator level and in matrix representations of the exchange operations. In particular, the whole Hilbert spaces for $n=2,3,4$ vortices are decomposed into subspaces according to representations of $\mathrm{U}(2)$, and the matrix forms of the exchange operations were obtained in these subspaces. We have found they have off-diagonal elements in some subspaces. This analysis is an extension of the previous one for the $\mathrm{U}(1)$ (one component) Dirac vortices [11]. By using a simple reduction of the $\mathrm{U}(2)$ results, we have indeed identified the same matrices as in the $\mathrm{U}(1)$ Dirac vortices. This way of identifying the U(1) structure is rather different from the one in the non-Abelian Majorana vortices where one can extract the $\mathrm{U}(1)$ part as a tensor product. It is not trivial at this point if a similar identification is possible in the Dirac vortices. Finally, we have found that the whole braid group contains a subgroup of genuine non-Abelian statistics for the exchange of the vortices with one Dirac fermion. The reason why non-Abelian statistics appears only from locally defined Dirac fermions is that the basis of the Hilbert space are entangled because of the representation of U(2) acting on the Dirac fermions. In this sense, the 'spatial' non-locality of Dirac fermions is not needed to have non-Abelian statistics but rather the non-locality, namely entanglement, in representations is needed.

\begin{tabular}{|c|c|c|c|c|}
\hline & \multicolumn{2}{|c|}{$\begin{array}{c}\text { Majorana vortices } \\
\hat{\gamma}_{k}=\hat{\gamma}_{k}^{\dagger} \\
\end{array}$} & \multicolumn{2}{|c|}{$\begin{array}{c}\text { Dirac vortices } \\
\hat{\psi}_{k} \neq \hat{\psi}_{k}^{\dagger} \\
\end{array}$} \\
\hline $\begin{array}{c}\text { \# of trapped fermions } \\
\text { and symmetry }\end{array}$ & $\begin{array}{c}1 \\
\text { non }\end{array}$ & $\begin{array}{cc}3, & 2 N-1 \\
\mathrm{SO}(3), & \mathrm{SO}(2 N-1)\end{array}$ & $\begin{array}{c}1 \\
\mathrm{U}(1)\end{array}$ & $\begin{array}{c}2 \\
\mathrm{U}(2)\end{array}$ \\
\hline examples & $p$-wave SC, etc & CFL phase of color SC & A-phase of ${ }^{3} \mathrm{He}$, etc & CFL phase of color SC, etc \\
\hline $\begin{array}{c}\text { dimension of } \\
n \text {-vortex system }\end{array}$ & $\begin{array}{l}2^{n / 2} \\
\text { for even } n\end{array}$ & $\begin{array}{c}\left(2^{3}\right)^{n / 2}, \quad\left(2^{2 N-1}\right)^{n / 2} \\
\text { for even } n\end{array}$ & $2^{n}$ & $\left(2^{2}\right)^{n}$ \\
\hline $\begin{array}{l}\text { representation } \\
\text { of braid group }\end{array}$ & $\begin{array}{c}\text { non-Abelian } \\
(\text { Ivanov matrices) }\end{array}$ & $\begin{array}{c}\text { non-Abelian } \\
(\text { Ivanov } \times \text { Coxeter })\end{array}$ & non-Abelian & non-Abelian \\
\hline $\begin{array}{l}\text { exchange } \\
\text { statistics }\end{array}$ & $\begin{array}{c}\text { non-Abelian } \\
\text { (Ivanov matrices) }\end{array}$ & $\begin{array}{c}\text { non-Abelian } \\
(\text { Ivanov } \times \text { Coxeter })\end{array}$ & Abelian & non-Abelian \\
\hline references & [6] & $\mathrm{SO}(3)[9], \mathrm{SO}(2 N-1)[10]$ & [11] & this paper \\
\hline
\end{tabular}

TABLE I: Summary of the results on non-Abelian representations of the braid group and non-Abelian statistics of Majorana/Dirac vortices with Abelian/non-Abelian symmetry. Note that the braid group acts on all particles while the non-Abelian statistics concerns only on identical particles. Multiple zero-energy fermions are in the vector representations of the groups shown in the table. In the Majorana vortices, the exchange matrices for a single fermion are called Ivanov matrices, and those for multiple fermions are decomposed into the Ivanov matrices and generators of the Coxeter group.

In Table I, we have summarized the results of Majorana/Dirac vortices with Abelian/non-Abelian symmetry. All of them exhibit non-Abelian representations of the braid group. The right most column corresponds to the present result. The exchange of Majorana fermions automatically gives non-Abelian statistics, while the exchange of Dirac fermions contains non-Abelian statistics when one exchanges identical particles, that is possible at least for U(2).

So far, we know only high-energy-physics examples of vortices with multiple Dirac fermions , i.e., non-Abelian vortices in dense QCD and supersymmetric QCD. As future studies, examples in condensed matter physics and experimental observation of the non-Abelian statistics in laboratory will be important. Applications to quantum computing should also be explored.

\section{Appendix A: Exchange matrices for U(1) Dirac vortices}

In this Appendix, we present the matrix representations of the exchange operators $\tau_{\ell}$ 's $(\ell=1, \cdots, n-1)$ for the U(1) Dirac vortices which was first obtained in Ref. [11] (below we omit the superscript "s" for notational simplicity). The Hilbert space of $n$ vortices, $\mathbb{H}^{(n)}$, is decomposed into subspaces $\mathbb{H}^{(n, f)}$ which are specified by the total fermion number $f$. 
In the case of $n=2\left(k\right.$-th and $(k+1)$-th vortices), we have only one exchange operation $T_{k}$. The Hilbert space $\mathbb{H}^{(2)}$ is decomposed into a direct sum of three subspaces: $\mathbb{H}^{(2,0)} \equiv\{|00\rangle\}, \mathbb{H}^{(2,1)} \equiv\{|10\rangle,|01\rangle\}$ and $\mathbb{H}^{(2,2)} \equiv\{|11\rangle\}$. Thus, $\mathbb{H}^{(2)}=\mathbb{H}^{(2,0)} \oplus \mathbb{H}^{(2,1)} \oplus \mathbb{H}^{(2,2)}$. Then, we obtain the matrix representations of $\hat{\tau}_{k}$ as

$$
\begin{aligned}
& \tau_{k}^{(2,0)}=1 \\
& \tau_{k}^{(2,1)}=\left(\begin{array}{cc}
0 & -1 \\
1 & 0
\end{array}\right), \\
& \tau_{k}^{(2,2)}=1 .
\end{aligned}
$$

In the case of $n=3\left(k\right.$-th, $(k+1)$-th and $(k+2)$-th vortices), we have two operations $T_{k}$ and $T_{k+1}$. The Hilbert space $\mathbb{H}^{(3)}$ is decomposed into a direct sum of $\mathbb{H}^{(3,0)} \equiv\{|000\rangle\}, \mathbb{H}^{(3,1)} \equiv\{|100\rangle,|010\rangle,|001\rangle\}, \mathbb{H}^{(3,2)} \equiv\{|110\rangle,|011\rangle,|101\rangle\}$ and $\mathbb{H}^{(3,3)} \equiv\{|111\rangle\}$. Namely, $\mathbb{H}^{(3)}=\mathbb{H}^{(3,0)} \oplus \mathbb{H}^{(3,1)} \oplus \mathbb{H}^{(3,2)} \oplus \mathbb{H}^{(3,3)}$. We thus have the matrix representations of $\hat{\tau}_{k}$ and $\hat{\tau}_{k+1}$ as

$$
\begin{aligned}
\tau_{k}^{(3,0)} & =\tau_{k+1}^{(3,0)}=1, \\
\tau_{k}^{(3,1)} & =\left(\begin{array}{ccc}
0 & -1 & 0 \\
1 & 0 & 0 \\
0 & 0 & 1
\end{array}\right), \tau_{k+1}^{(3,1)}=\left(\begin{array}{ccc}
1 & 0 & 0 \\
0 & 0 & -1 \\
0 & 1 & 0
\end{array}\right), \\
\tau_{k}^{(3,2)} & =\left(\begin{array}{ccc}
1 & 0 & 0 \\
0 & 0 & 1 \\
0 & -1 & 0
\end{array}\right), \tau_{k+1}^{(3,2)}=\left(\begin{array}{ccc}
0 & 0 & -1 \\
0 & 1 & 0 \\
1 & 0 & 0
\end{array}\right), \\
\tau_{k}^{(3,3)} & =\tau_{k+1}^{(3,3)}=1 .
\end{aligned}
$$

In the case of $n=4$ ( $k$-th, $(k+1)$-th, $(k+2)$-th and $(k+3)$-th vortices), we have three operations $T_{k}$, $T_{k+1}$ and $T_{k+2}$. The Hilbert space $\mathbb{H}^{(4)}$ is decomposed into a direct sum of five sectors: $\mathbb{H}^{(4,0)} \equiv\{|0000\rangle\}$, $\mathbb{H}^{(4,1)} \equiv\{|1000\rangle,|0100\rangle,|0010\rangle,|0001\rangle\}, \mathbb{H}^{(4,2)} \equiv\{|1100\rangle,|1010\rangle,|1001\rangle,|0110\rangle,|0101\rangle,|0011\rangle\}, \mathbb{H}^{(4,3)} \equiv\{|1110\rangle$, $|1101\rangle,|1011\rangle,|0111\rangle\}$ and $\mathbb{H}^{(4,4)} \equiv\{|1111\rangle\}$. Namely, $\mathbb{H}^{(4)}=\mathbb{H}^{(4,0)} \oplus \mathbb{H}^{(4,1)} \oplus \mathbb{H}^{(4,2)} \oplus \mathbb{H}^{(4,3)} \oplus \mathbb{H}^{(4,4)}$. The matrix representations of $\hat{\tau}_{k}, \hat{\tau}_{k+1}$ and $\hat{\tau}_{k+2}$ are

$$
\begin{aligned}
& \tau_{k}^{(4,0)}=\tau_{k+1}^{(4,0)}=\tau_{k+2}^{(4,0)}=1, \\
& \tau_{k}^{(4,1)}=\left(\begin{array}{cccc}
0 & -1 & 0 & 0 \\
1 & 0 & 0 & 0 \\
0 & 0 & 1 & 0 \\
0 & 0 & 0 & 1
\end{array}\right), \tau_{k+1}^{(4,1)}=\left(\begin{array}{cccc}
1 & 0 & 0 & 0 \\
0 & 0 & -1 & 0 \\
0 & 1 & 0 & 0 \\
0 & 0 & 0 & 1
\end{array}\right), \tau_{k+2}^{(4,1)}=\left(\begin{array}{cccc}
1 & 0 & 0 & 0 \\
0 & 1 & 0 & 0 \\
0 & 0 & 0 & -1 \\
0 & 0 & 1 & 0
\end{array}\right), \\
& \tau_{k}^{(4,2)}=\left(\begin{array}{cccccc}
1 & 0 & 0 & 0 & 0 & 0 \\
0 & 0 & 0 & -1 & 0 & 0 \\
0 & 0 & 0 & 0 & -1 & 0 \\
0 & 1 & 0 & 0 & 0 & 0 \\
0 & 0 & 1 & 0 & 0 & 0 \\
0 & 0 & 0 & 0 & 0 & 1
\end{array}\right), \tau_{k+1}^{(4,2)}=\left(\begin{array}{cccccc}
0 & -1 & 0 & 0 & 0 & 0 \\
1 & 0 & 0 & 0 & 0 & 0 \\
0 & 0 & 1 & 0 & 0 & 0 \\
0 & 0 & 0 & 1 & 0 & 0 \\
0 & 0 & 0 & 0 & 0 & -1 \\
0 & 0 & 0 & 0 & 1 & 0
\end{array}\right), \tau_{k+2}^{(4,2)}=\left(\begin{array}{cccccc}
1 & 0 & 0 & 0 & 0 & 0 \\
0 & 0 & -1 & 0 & 0 & 0 \\
0 & 1 & 0 & 0 & 0 & 0 \\
0 & 0 & 0 & 0 & -1 & 0 \\
0 & 0 & 0 & 1 & 0 & 0 \\
0 & 0 & 0 & 0 & 0 & 1
\end{array}\right), \\
& \tau_{k}^{(4,3)}=\left(\begin{array}{cccc}
1 & 0 & 0 & 0 \\
0 & 1 & 0 & 0 \\
0 & 0 & 0 & -1 \\
0 & 0 & 1 & 0
\end{array}\right), \tau_{k+1}^{(4,3)}=\left(\begin{array}{cccc}
1 & 0 & 0 & 0 \\
0 & 0 & -1 & 0 \\
0 & 1 & 0 & 0 \\
0 & 0 & 0 & 1
\end{array}\right), \tau_{k+2}^{(4,3)}=\left(\begin{array}{cccc}
0 & -1 & 0 & 0 \\
1 & 0 & 0 & 0 \\
0 & 0 & 1 & 0 \\
0 & 0 & 0 & 1
\end{array}\right) \text {, } \\
& \tau_{k}^{(4,4)}=\tau_{k+1}^{(4,4)}=\tau_{k+2}^{(4,4)}=1 .
\end{aligned}
$$

\section{Appendix B: The case of $n=4$ for the U(2) Dirac vortices}

We consider the system of $n=4$ vortices, which is an ensemble of $k$-th, $(k+1)$-th, $(k+2)$-th, and $(k+3)$-th vortices. 
The Hilbert space is constructed straightforwardly from the $n=2$ and $n=3$ cases. The representations of the $\mathrm{U}(2)$ symmetry are decomposed as

$$
\begin{aligned}
& \left(\mathbf{1}_{0}+\mathbf{2}_{1}+\mathbf{1}_{2}\right)_{\text {vortex } k} \otimes\left(\mathbf{1}_{0}+\mathbf{2}_{1}+\mathbf{1}_{2}\right)_{\text {vortex } k+1} \otimes\left(\mathbf{1}_{0}+\mathbf{2}_{1}+\mathbf{1}_{2}\right)_{\text {vortex } k+2} \otimes\left(\mathbf{1}_{0}+\mathbf{2}_{1}+\mathbf{1}_{2}\right)_{\text {vortex } k+3} \\
& =\mathbf{1}_{0000}+\mathbf{1}_{0020}+\mathbf{1}_{0002}+\mathbf{1}_{2000}+\mathbf{1}_{0200} \\
& +\mathbf{1}_{0011}+\mathbf{1}_{1100}+\mathbf{1}_{1010}+\mathbf{1}_{1001}+\mathbf{1}_{0110}+\mathbf{1}_{0101} \\
& +\mathbf{1}_{0022}+\mathbf{1}_{2020}+\mathbf{1}_{2002}+\mathbf{1}_{0220}+\mathbf{1}_{0202}+\mathbf{1}_{2200} \\
& +\mathbf{1}_{1120}+\mathbf{1}_{1102}+\mathbf{1}_{2011}+\mathbf{1}_{0211}+\mathbf{1}_{1021}+\mathbf{1}_{1012}+\mathbf{1}_{0121}+\mathbf{1}_{0112}+\mathbf{1}_{2110}+\mathbf{1}_{2101}+\mathbf{1}_{1210}+\mathbf{1}_{1201} \\
& +\mathbf{1}_{2022}+\mathbf{1}_{0222}+\mathbf{1}_{2220}+\mathbf{1}_{2202} \\
& +\mathbf{1}_{\mathrm{AA} \underline{11} \underline{11}}+\mathbf{1}_{\mathrm{SS} \underline{11} \underline{11}} \\
& +\mathbf{1}_{1122}+\mathbf{1}_{2211}+\mathbf{1}_{2121}+\mathbf{1}_{2112}+\mathbf{1}_{1221}+\mathbf{1}_{1212} \\
& +\mathbf{1}_{2222} \\
& +\mathbf{2}_{1000}+\mathbf{2}_{0100}+\mathbf{2}_{0010}+\mathbf{2}_{0001} \\
& +\mathbf{2}_{0021}+\mathbf{2}_{0012}+\mathbf{2}_{2010}+\mathbf{2}_{2001}+\mathbf{2}_{0210}+\mathbf{2}_{0201}+\mathbf{2}_{1020}+\mathbf{2}_{1002}+\mathbf{2}_{0120}+\mathbf{2}_{0102}+\mathbf{2}_{2100}+\mathbf{2}_{1200} \\
& +\mathbf{2}_{\mathrm{A} \underline{11} 10}+\mathbf{2}_{\mathrm{A} \underline{1101}}+\mathbf{2}_{\mathrm{A} 10 \underline{11}}+\mathbf{2}_{\mathrm{S} 10 \underline{11}}+\mathbf{2}_{\mathrm{A} 01 \underline{11}}+\mathbf{2}_{\mathrm{S} 01 \underline{11}}+\mathbf{2}_{\mathrm{S} \underline{11} 10}+\mathbf{2}_{\mathrm{S} \underline{1101}} \\
& +\mathbf{2}_{2021}+\mathbf{2}_{2012}+\mathbf{2}_{0221}+\mathbf{2}_{0212}+\mathbf{2}_{2210}+\mathbf{2}_{2201}+\mathbf{2}_{1022}+\mathbf{2}_{0122}+\mathbf{2}_{2120}+\mathbf{2}_{2102}+\mathbf{2}_{1220}+\mathbf{2}_{1202} \\
& +\mathbf{2}_{\mathrm{A} 1121}+\mathbf{2}_{\mathrm{A} \underline{1112}}+\mathbf{2}_{\mathrm{A} 21 \underline{11}}+\mathbf{2}_{\mathrm{S} 21 \underline{11}}+\mathbf{2}_{\mathrm{A} 12 \underline{11}}+\mathbf{2}_{\mathrm{S} 12 \underline{11}}+\mathbf{2}_{\mathrm{S} \underline{1121}}+\mathbf{2}_{\mathrm{S} \underline{111} 2} \\
& +\mathbf{2}_{2221}+\mathbf{2}_{2212}+\mathbf{2}_{2122}+\mathbf{2}_{1222} \\
& +\mathbf{3}_{1100}+\mathbf{3}_{1010}+\mathbf{3}_{1001}+\mathbf{3}_{0110}+\mathbf{3}_{0101}+\mathbf{3}_{0011} \\
& +\mathbf{3}_{2011}+\mathbf{3}_{0211}+\mathbf{3}_{1021}+\mathbf{3}_{1012}+\mathbf{3}_{0121}+\mathbf{3}_{0112}+\mathbf{3}_{2110}+\mathbf{3}_{2101}+\mathbf{3}_{1210}+\mathbf{3}_{1201}+\mathbf{3}_{1120}+\mathbf{3}_{1102} \\
& +\mathbf{3}_{\mathrm{AS} \underline{11} \underline{11}}+\mathbf{3}_{\mathrm{SA} \underline{11} \underline{11}}+\mathbf{3}_{\mathrm{SS} \underline{11} \underline{11}} \\
& +\mathbf{3}_{2211}+\mathbf{3}_{2121}+\mathbf{3}_{2112}+\mathbf{3}_{1221}+\mathbf{3}_{1212}+\mathbf{3}_{1122} \\
& +4_{\mathrm{S} 1110}+4_{\mathrm{S} \underline{1101}}+4_{\mathrm{S} 10 \underline{11}}+4_{\mathrm{S} 01 \underline{1}} \\
& +4_{\mathrm{S} 21 \underline{11}}+4_{\mathrm{S} 12 \underline{11}}+4_{\mathrm{S} \underline{1121}}+4_{\mathrm{S} \underline{11} 12} \\
& +5_{\mathrm{S}}^{11} \underline{11}
\end{aligned}
$$

where the subscript $n_{k} n_{k+1} n_{k+2} n_{k+3}\left(n_{k}, n_{k+1}, n_{k+2}, n_{k+3}=0,1,2\right)$ denotes the number of the Dirac fermions, $n_{k}$, $n_{k+1} n_{k+2}$ and $n_{k+3}$, at the $k$-th, $(k+1)$-th, $(k+2)$-th and $(k+3)$-th vortices, respectively. One obtains the basis states of the Hilbert space by applying $\hat{\psi}_{\ell}^{a \dagger}(\ell=k, k+1, k+2, k+3$ and $a=1,2)$ successively to the Fock vacuum $|0\rangle$ defined by $\hat{\psi}_{\ell}^{a}|0\rangle=0$ for all $\ell$ and $a=1,2$.

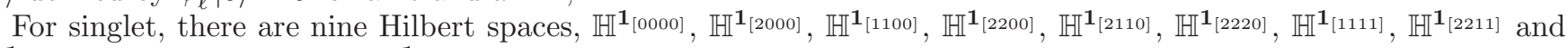

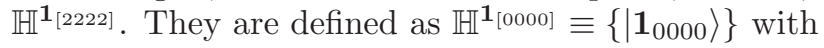

$$
\left|\mathbf{1}_{0000}\right\rangle \equiv|0\rangle,
$$

$\mathbb{H}^{\mathbf{1}_{[2000]}} \equiv\left\{\left|\mathbf{1}_{0020}\right\rangle,\left|\mathbf{1}_{0002}\right\rangle,\left|\mathbf{1}_{2000}\right\rangle,\left|\mathbf{1}_{0200}\right\rangle\right\}$ with

$$
\begin{aligned}
\left|\mathbf{1}_{0020}\right\rangle & \equiv \hat{\psi}_{k+2}^{1 \dagger} \hat{\psi}_{k+2}^{2 \dagger}|0\rangle, \\
\left|\mathbf{1}_{0002}\right\rangle & \equiv \hat{\psi}_{k+3}^{1 \dagger} \hat{\psi}_{k+3}^{2 \dagger}|0\rangle, \\
\left|\mathbf{1}_{2000}\right\rangle & \equiv \hat{\psi}_{k}^{1 \dagger} \hat{\psi}_{k}^{2 \dagger}|0\rangle, \\
\left|\mathbf{1}_{0200}\right\rangle & \equiv \hat{\psi}_{k+1}^{1 \dagger} \hat{\psi}_{k+1}^{2 \dagger}|0\rangle,
\end{aligned}
$$

$\mathbb{H}^{\mathbf{1}_{[1100]}} \equiv\left\{\left|\mathbf{1}_{0011}\right\rangle,\left|\mathbf{1}_{1100}\right\rangle,\left|\mathbf{1}_{1010}\right\rangle,\left|\mathbf{1}_{1001}\right\rangle,\left|\mathbf{1}_{0110}\right\rangle,\left|\mathbf{1}_{0101}\right\rangle\right\}$ with

$$
\begin{aligned}
\left|\mathbf{1}_{0011}\right\rangle & \equiv \frac{1}{\sqrt{2}}\left(\hat{\psi}_{k+2}^{1 \dagger} \hat{\psi}_{k+3}^{2 \dagger}-\hat{\psi}_{k+2}^{2 \dagger} \hat{\psi}_{k+3}^{1 \dagger}\right)|0\rangle, \\
\left|\mathbf{1}_{1100}\right\rangle & \equiv \frac{1}{\sqrt{2}}\left(\hat{\psi}_{k}^{1 \dagger} \hat{\psi}_{k+1}^{2 \dagger}-\hat{\psi}_{k}^{2 \dagger} \hat{\psi}_{k+1}^{1 \dagger}\right)|0\rangle, \\
\left|\mathbf{1}_{1010}\right\rangle & \equiv \frac{1}{\sqrt{2}}\left(\hat{\psi}_{k}^{1 \dagger} \hat{\psi}_{k+2}^{2 \dagger}-\hat{\psi}_{k}^{2 \dagger} \hat{\psi}_{k+2}^{1 \dagger}\right)|0\rangle, \\
\left|\mathbf{1}_{1001}\right\rangle & \equiv \frac{1}{\sqrt{2}}\left(\hat{\psi}_{k}^{1 \dagger} \hat{\psi}_{k+3}^{2 \dagger}-\hat{\psi}_{k}^{2 \dagger} \hat{\psi}_{k+3}^{1 \dagger}\right)|0\rangle,
\end{aligned}
$$




$$
\begin{aligned}
& \left|\mathbf{1}_{0110}\right\rangle \equiv \frac{1}{\sqrt{2}}\left(\hat{\psi}_{k+1}^{1 \dagger} \hat{\psi}_{k+2}^{2 \dagger}-\hat{\psi}_{k+1}^{2 \dagger} \hat{\psi}_{k+2}^{1 \dagger}\right)|0\rangle, \\
& \left|\mathbf{1}_{0101}\right\rangle \equiv \frac{1}{\sqrt{2}}\left(\hat{\psi}_{k+1}^{1 \dagger} \hat{\psi}_{k+3}^{2 \dagger}-\hat{\psi}_{k+1}^{2 \dagger} \hat{\psi}_{k+3}^{1 \dagger}\right)|0\rangle,
\end{aligned}
$$

$\mathbb{H}^{\mathbf{1}_{[2200]}} \equiv\left\{\left|\mathbf{1}_{0022}\right\rangle,\left|\mathbf{1}_{2020}\right\rangle,\left|\mathbf{1}_{2002}\right\rangle,\left|\mathbf{1}_{0220}\right\rangle,\left|\mathbf{1}_{0202}\right\rangle,\left|\mathbf{1}_{2200}\right\rangle\right\}$ with

$$
\begin{aligned}
\left|\mathbf{1}_{0022}\right\rangle & \equiv \hat{\psi}_{k+2}^{1 \dagger} \hat{\psi}_{k+2}^{2 \dagger} \hat{\psi}_{k+3}^{1 \dagger} \hat{\psi}_{k+3}^{2 \dagger}|0\rangle, \\
\left|\mathbf{1}_{2020}\right\rangle & \equiv \hat{\psi}_{k}^{1 \dagger} \hat{\psi}_{k}^{2 \dagger} \hat{\psi}_{k+2}^{1 \dagger} \hat{\psi}_{k+2}^{2 \dagger}|0\rangle, \\
\left|\mathbf{1}_{2002}\right\rangle & \equiv \hat{\psi}_{k}^{1 \dagger} \hat{\psi}_{k}^{2 \dagger} \hat{\psi}_{k+3}^{1 \dagger} \hat{\psi}_{k+3}^{2 \dagger}|0\rangle, \\
\left|\mathbf{1}_{0220}\right\rangle & \equiv \hat{\psi}_{k+1}^{1 \dagger} \hat{\psi}_{k+1}^{2 \dagger} \hat{\psi}_{k+2}^{1 \dagger} \hat{\psi}_{k+2}^{2 \dagger}|0\rangle, \\
\left|\mathbf{1}_{0202}\right\rangle & \equiv \hat{\psi}_{k+1}^{1 \dagger} \hat{\psi}_{k+1}^{2 \dagger} \hat{\psi}_{k+3}^{1 \dagger} \hat{\psi}_{k+3}^{2 \dagger}|0\rangle, \\
\left|\mathbf{1}_{2200}\right\rangle & \equiv \hat{\psi}_{k}^{1 \dagger} \hat{\psi}_{k}^{2 \dagger} \hat{\psi}_{k+1}^{1 \dagger} \hat{\psi}_{k+1}^{2 \dagger}|0\rangle,
\end{aligned}
$$

$\mathbb{H}^{\mathbf{1}^{[2110]}} \equiv\left\{\left|\mathbf{1}_{1120}\right\rangle,\left|\mathbf{1}_{1102}\right\rangle,\left|\mathbf{1}_{2011}\right\rangle,\left|\mathbf{1}_{0211}\right\rangle,\left|\mathbf{1}_{1021}\right\rangle,\left|\mathbf{1}_{1012}\right\rangle,\left|\mathbf{1}_{0121}\right\rangle,\left|\mathbf{1}_{0112}\right\rangle,\left|\mathbf{1}_{2110}\right\rangle,\left|\mathbf{1}_{2101}\right\rangle,\left|\mathbf{1}_{1210}\right\rangle,\left|\mathbf{1}_{1201}\right\rangle\right\}$ with

$$
\begin{aligned}
\left|\mathbf{1}_{1120}\right\rangle & \equiv \frac{1}{\sqrt{2}}\left(\hat{\psi}_{k}^{1 \dagger} \hat{\psi}_{k+1}^{2 \dagger}-\hat{\psi}_{k}^{2 \dagger} \hat{\psi}_{k+1}^{1 \dagger}\right) \hat{\psi}_{k+2}^{1 \dagger} \hat{\psi}_{k+2}^{2 \dagger}|0\rangle, \\
\left|\mathbf{1}_{1102}\right\rangle & \equiv \frac{1}{\sqrt{2}}\left(\hat{\psi}_{k}^{1 \dagger} \hat{\psi}_{k+1}^{2 \dagger}-\hat{\psi}_{k}^{2 \dagger} \hat{\psi}_{k+1}^{1 \dagger}\right) \hat{\psi}_{k+3}^{1 \dagger} \hat{\psi}_{k+3}^{2 \dagger}|0\rangle, \\
\left|\mathbf{1}_{2011}\right\rangle & \equiv \frac{1}{\sqrt{2}} \hat{\psi}_{k}^{1 \dagger} \hat{\psi}_{k}^{2 \dagger}\left(\hat{\psi}_{k+2}^{1 \dagger} \hat{\psi}_{k+3}^{2 \dagger}-\hat{\psi}_{k+2}^{2 \dagger} \hat{\psi}_{k+3}^{1 \dagger}\right)|0\rangle, \\
\left|\mathbf{1}_{0211}\right\rangle & \equiv \frac{1}{\sqrt{2}} \hat{\psi}_{k+1}^{1 \dagger} \hat{\psi}_{k+1}^{2 \dagger}\left(\hat{\psi}_{k+2}^{1 \dagger} \hat{\psi}_{k+3}^{2 \dagger}-\hat{\psi}_{k+2}^{2 \dagger} \hat{\psi}_{k+3}^{1 \dagger}\right)|0\rangle, \\
\left|\mathbf{1}_{1021}\right\rangle & \equiv \frac{1}{\sqrt{2}}\left(\hat{\psi}_{k}^{1 \dagger} \hat{\psi}_{k+3}^{2 \dagger}-\hat{\psi}_{k}^{2 \dagger} \hat{\psi}_{k+3}^{1 \dagger}\right) \hat{\psi}_{k+2}^{1 \dagger} \hat{\psi}_{k+2}^{2 \dagger}|0\rangle, \\
\left|\mathbf{1}_{1012}\right\rangle & \equiv \frac{1}{\sqrt{2}}\left(\hat{\psi}_{k}^{1 \dagger} \hat{\psi}_{k+2}^{2 \dagger}-\hat{\psi}_{k}^{2 \dagger} \hat{\psi}_{k+2}^{1 \dagger}\right) \hat{\psi}_{k+3}^{1 \dagger} \hat{\psi}_{k+3}^{2 \dagger}|0\rangle, \\
\left|\mathbf{1}_{0121}\right\rangle & \equiv \frac{1}{\sqrt{2}}\left(\hat{\psi}_{k+1}^{1 \dagger} \hat{\psi}_{k+3}^{2 \dagger}-\hat{\psi}_{k+1}^{2 \dagger} \hat{\psi}_{k+3}^{1 \dagger}\right) \hat{\psi}_{k+2}^{1 \dagger} \hat{\psi}_{k+2}^{2 \dagger}|0\rangle, \\
\left|\mathbf{1}_{0112}\right\rangle & \equiv \frac{1}{\sqrt{2}}\left(\hat{\psi}_{k+1}^{1 \dagger} \hat{\psi}_{k+2}^{2 \dagger}-\hat{\psi}_{k+1}^{2 \dagger} \hat{\psi}_{k+2}^{1 \dagger}\right) \hat{\psi}_{k+3}^{1 \dagger} \hat{\psi}_{k+3}^{2 \dagger}|0\rangle, \\
\left|\mathbf{1}_{2110}\right\rangle & \equiv \frac{1}{\sqrt{2}} \hat{\psi}_{k}^{1 \dagger} \hat{\psi}_{k}^{2 \dagger}\left(\hat{\psi}_{k+1}^{1 \dagger} \hat{\psi}_{k+2}^{2 \dagger}-\hat{\psi}_{k+1}^{2 \dagger} \hat{\psi}_{k+2}^{1 \dagger}\right)|0\rangle, \\
\left|\mathbf{1}_{2101}\right\rangle & \equiv \frac{1}{\sqrt{2}} \hat{\psi}_{k}^{1 \dagger} \hat{\psi}_{k}^{2 \dagger}\left(\hat{\psi}_{k+1}^{1 \dagger} \hat{\psi}_{k+3}^{2 \dagger}-\hat{\psi}_{k+1}^{2 \dagger} \hat{\psi}_{k+3}^{1 \dagger}\right)|0\rangle, \\
\left|\mathbf{1}_{1210}\right\rangle & \equiv \frac{1}{\sqrt{2}}\left(\hat{\psi}_{k}^{1 \dagger} \hat{\psi}_{k+2}^{2 \dagger}-\hat{\psi}_{k}^{2 \dagger} \hat{\psi}_{k+2}^{1 \dagger}\right) \hat{\psi}_{k+1}^{1 \dagger} \hat{\psi}_{k+1}^{2 \dagger}|0\rangle, \\
\left|\mathbf{1}_{1201}\right\rangle & \equiv \frac{1}{\sqrt{2}}\left(\hat{\psi}_{k}^{1 \dagger} \hat{\psi}_{k+3}^{2 \dagger}-\hat{\psi}_{k}^{2 \dagger} \hat{\psi}_{k+3}^{1 \dagger}\right) \hat{\psi}_{k+1}^{1 \dagger} \hat{\psi}_{k+1}^{2 \dagger}|0\rangle,
\end{aligned}
$$

$\mathbb{H}^{\left[\mathbf{1}^{[220]}\right.} \equiv\left\{\left|\mathbf{1}_{2022}\right\rangle,\left|\mathbf{1}_{0222}\right\rangle,\left|\mathbf{1}_{2220}\right\rangle,\left|\mathbf{1}_{2202}\right\rangle\right\}$ with

$$
\begin{aligned}
\left|\mathbf{1}_{2022}\right\rangle & \equiv \hat{\psi}_{k}^{1 \dagger} \hat{\psi}_{k}^{2 \dagger} \hat{\psi}_{k+2}^{1 \dagger} \hat{\psi}_{k+2}^{2 \dagger} \hat{\psi}_{k+3}^{1 \dagger} \hat{\psi}_{k+3}^{2 \dagger}|0\rangle, \\
\left|\mathbf{1}_{0222}\right\rangle & \equiv \hat{\psi}_{k+1}^{1 \dagger} \hat{\psi}_{k+1}^{2 \dagger} \hat{\psi}_{k+2}^{1 \dagger} \hat{\psi}_{k+2}^{2 \dagger} \hat{\psi}_{k+3}^{1 \dagger} \hat{\psi}_{k+3}^{2 \dagger}|0\rangle, \\
\left|\mathbf{1}_{2220}\right\rangle & \equiv \hat{\psi}_{k}^{1 \dagger} \hat{\psi}_{k}^{2 \dagger} \hat{\psi}_{k+1}^{1 \dagger} \hat{\psi}_{k+1}^{2 \dagger} \hat{\psi}_{k+2}^{1 \dagger} \hat{\psi}_{k+2}^{2 \dagger}|0\rangle, \\
\left|\mathbf{1}_{2202}\right\rangle & \equiv \hat{\psi}_{k}^{1 \dagger} \hat{\psi}_{k}^{2 \dagger} \hat{\psi}_{k+1}^{1 \dagger} \hat{\psi}_{k+1}^{2 \dagger} \hat{\psi}_{k+3}^{1 \dagger} \hat{\psi}_{k+3}^{2 \dagger}|0\rangle,
\end{aligned}
$$

$\mathbb{H}^{\mathbf{1}_{[111]}} \equiv\left\{\left|\mathbf{1}_{\mathrm{AA} \underline{11} \underline{11}}\right\rangle,\left|\mathbf{1}_{\mathrm{SS}} \underline{11} \underline{11}\right\rangle\right\}$ with

$$
\left|\mathbf{1}_{\mathrm{AA}} \underline{11} \underline{11}\right\rangle \equiv \frac{1}{2}\left(\hat{\psi}_{k}^{1 \dagger} \hat{\psi}_{k+1}^{2 \dagger}-\hat{\psi}_{k}^{2 \dagger} \hat{\psi}_{k+1}^{1 \dagger}\right)\left(\hat{\psi}_{k+2}^{1 \dagger} \hat{\psi}_{k+3}^{2 \dagger}-\hat{\psi}_{k+2}^{2 \dagger} \hat{\psi}_{k+3}^{1 \dagger}\right)|0\rangle,
$$




$$
\left|\mathbf{1}_{\mathrm{SS} \underline{11} \underline{11}}\right\rangle \equiv \frac{1}{\sqrt{3}}\left\{\hat{\psi}_{k}^{1 \dagger} \hat{\psi}_{k+1}^{1 \dagger} \hat{\psi}_{k+2}^{2 \dagger} \hat{\psi}_{k+3}^{2 \dagger}-\frac{1}{2}\left(\hat{\psi}_{k}^{1 \dagger} \hat{\psi}_{k+1}^{2 \dagger}+\hat{\psi}_{k}^{2 \dagger} \hat{\psi}_{k+1}^{1 \dagger}\right)\left(\hat{\psi}_{k+2}^{1 \dagger} \hat{\psi}_{k+3}^{2 \dagger}+\hat{\psi}_{k+2}^{2 \dagger} \hat{\psi}_{k+3}^{1 \dagger}\right)+\hat{\psi}_{k}^{2 \dagger} \hat{\psi}_{k+1}^{2 \dagger} \hat{\psi}_{k+2}^{1 \dagger} \hat{\psi}_{k+3}^{1 \dagger}\right\}|0\rangle
$$

with a notation A (S) for an antisymmetric (symmetric) combination in first and second pair of indices with the underline in the $k$-th and $(k+1)$-th vortices and in the $(k+2)$-th and $(k+3)$-th vortices. $\mathbb{H}^{\mathbf{1}^{[2211]}} \equiv$ $\left\{\left|\mathbf{1}_{1122}\right\rangle,\left|\mathbf{1}_{2211}\right\rangle,\left|\mathbf{1}_{2121}\right\rangle,\left|\mathbf{1}_{2112}\right\rangle,\left|\mathbf{1}_{1221}\right\rangle,\left|\mathbf{1}_{1212}\right\rangle\right\}$ with

$$
\begin{aligned}
\left|\mathbf{1}_{1122}\right\rangle & \equiv \frac{1}{\sqrt{2}}\left(\hat{\psi}_{k}^{1 \dagger} \hat{\psi}_{k+1}^{2 \dagger}-\hat{\psi}_{k}^{2 \dagger} \hat{\psi}_{k+1}^{1 \dagger}\right) \hat{\psi}_{k+2}^{1 \dagger} \hat{\psi}_{k+2}^{2 \dagger} \hat{\psi}_{k+3}^{1 \dagger} \hat{\psi}_{k+3}^{2 \dagger}|0\rangle, \\
\left|\mathbf{1}_{2211}\right\rangle & \equiv \frac{1}{\sqrt{2}} \hat{\psi}_{k}^{1 \dagger} \hat{\psi}_{k}^{2 \dagger} \hat{\psi}_{k+1}^{1 \dagger} \hat{\psi}_{k+1}^{2 \dagger}\left(\hat{\psi}_{k+2}^{1 \dagger} \hat{\psi}_{k+3}^{2 \dagger}-\hat{\psi}_{k+2}^{2 \dagger} \hat{\psi}_{k+3}^{1 \dagger}\right)|0\rangle, \\
\left|\mathbf{1}_{2121}\right\rangle & \equiv \frac{1}{\sqrt{2}} \hat{\psi}_{k}^{1 \dagger} \hat{\psi}_{k}^{2 \dagger}\left(\hat{\psi}_{k+1}^{1 \dagger} \hat{\psi}_{k+3}^{2 \dagger}-\hat{\psi}_{k+1}^{2 \dagger} \hat{\psi}_{k+3}^{1 \dagger}\right) \hat{\psi}_{k+2}^{1 \dagger} \hat{\psi}_{k+2}^{2 \dagger}|0\rangle, \\
\left|\mathbf{1}_{2112}\right\rangle & \equiv \frac{1}{\sqrt{2}} \hat{\psi}_{k}^{1 \dagger} \hat{\psi}_{k}^{2 \dagger}\left(\hat{\psi}_{k+1}^{1 \dagger} \hat{\psi}_{k+2}^{2 \dagger}-\hat{\psi}_{k+1}^{2 \dagger} \hat{\psi}_{k+2}^{1 \dagger}\right) \hat{\psi}_{k+3}^{1 \dagger} \hat{\psi}_{k+3}^{2 \dagger}|0\rangle, \\
\left|\mathbf{1}_{1221}\right\rangle & \equiv \frac{1}{\sqrt{2}}\left(\hat{\psi}_{k}^{1 \dagger} \hat{\psi}_{k+3}^{2 \dagger}-\hat{\psi}_{k}^{2 \dagger} \hat{\psi}_{k+3}^{1 \dagger}\right) \hat{\psi}_{k+1}^{1 \dagger} \hat{\psi}_{k+1}^{2 \dagger} \hat{\psi}_{k+2}^{1 \dagger} \hat{\psi}_{k+2}^{2 \dagger}|0\rangle, \\
\left|\mathbf{1}_{1212}\right\rangle & \equiv \frac{1}{\sqrt{2}}\left(\hat{\psi}_{k}^{1 \dagger} \hat{\psi}_{k+2}^{2 \dagger}-\hat{\psi}_{k}^{2 \dagger} \hat{\psi}_{k+2}^{1 \dagger}\right) \hat{\psi}_{k+1}^{1 \dagger} \hat{\psi}_{k+1}^{2 \dagger} \hat{\psi}_{k+3}^{1 \dagger} \hat{\psi}_{k+3}^{2 \dagger}|0\rangle,
\end{aligned}
$$

and $\mathbb{H}^{\mathbf{1}_{[222]}} \equiv\left\{\left|\mathbf{1}_{2222}\right\rangle\right\}$ with

$$
\left|\mathbf{1}_{2222}\right\rangle \equiv \hat{\psi}_{k}^{1 \dagger} \hat{\psi}_{k}^{2 \dagger} \hat{\psi}_{k+1}^{1 \dagger} \hat{\psi}_{k+1}^{2 \dagger} \hat{\psi}_{k+2}^{1 \dagger} \hat{\psi}_{k+2}^{2 \dagger} \hat{\psi}_{k+3}^{1 \dagger} \hat{\psi}_{k+3}^{2 \dagger}|0\rangle .
$$

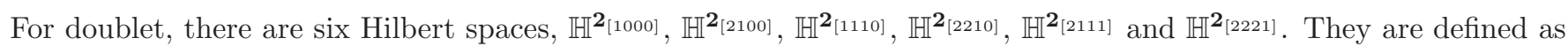
$\mathbb{H}^{\mathbf{2}_{[1000]}} \equiv\left\{\left|\mathbf{2}_{1000}\right\rangle,\left|\mathbf{2}_{0100}\right\rangle,\left|\mathbf{2}_{0010}\right\rangle,\left|\mathbf{2}_{0001}\right\rangle\right\}$ with

$$
\begin{aligned}
&\left|\mathbf{2}_{1000}\right\rangle \equiv\left(\begin{array}{c}
\hat{\psi}_{k}^{1 \dagger} \\
\hat{\psi}_{k}^{2 \dagger}
\end{array}\right)|0\rangle, \\
&\left|\mathbf{2}_{0100}\right\rangle \equiv\left(\begin{array}{c}
\hat{\psi}_{k+1}^{1 \dagger} \\
\hat{\psi}_{k+1}^{\dagger \dagger}
\end{array}\right)|0\rangle, \\
&\left|\mathbf{2}_{0010}\right\rangle \equiv\left(\begin{array}{c}
\hat{\psi}_{k+2}^{1 \dagger} \\
\hat{\psi}_{k+2}^{2 \dagger}
\end{array}\right)|0\rangle, \\
&\left|\mathbf{2}_{0001}\right\rangle \equiv\left(\begin{array}{c}
\hat{\psi}_{k+3}^{1 \dagger} \\
\hat{\psi}_{k+3}^{2 \dagger}
\end{array}\right)|0\rangle,
\end{aligned}
$$

$\mathbb{H}^{\mathbf{2}_{[2100]}} \equiv\left\{\left|\mathbf{2}_{0021}\right\rangle,\left|\mathbf{2}_{0012}\right\rangle,\left|\mathbf{2}_{2010}\right\rangle,\left|\mathbf{2}_{2001}\right\rangle,\left|\mathbf{2}_{0210}\right\rangle,\left|\mathbf{2}_{0201}\right\rangle,\left|\mathbf{2}_{1020}\right\rangle,\left|\mathbf{2}_{1002}\right\rangle,\left|\mathbf{2}_{0120}\right\rangle,\left|\mathbf{2}_{0102}\right\rangle,\left|\mathbf{2}_{2100}\right\rangle,\left|\mathbf{2}_{1200}\right\rangle\right\}$ with

$$
\begin{aligned}
\left|\mathbf{2}_{0021}\right\rangle & \equiv \hat{\psi}_{k+2}^{1 \dagger} \hat{\psi}_{k+2}^{2 \dagger}\left(\begin{array}{c}
\hat{\psi}_{k+3}^{1 \dagger} \\
\hat{\psi}_{k+3}^{2 \dagger}
\end{array}\right)|0\rangle, \\
\left|\mathbf{2}_{0012}\right\rangle & \equiv\left(\begin{array}{c}
\hat{\psi}_{k+2}^{1 \dagger} \\
\hat{\psi}_{k+2}^{2 \dagger}
\end{array}\right) \hat{\psi}_{k+3}^{1 \dagger} \hat{\psi}_{k+3}^{2 \dagger}|0\rangle, \\
\left|\mathbf{2}_{2010}\right\rangle & \equiv \hat{\psi}_{k}^{1 \dagger} \hat{\psi}_{k}^{2 \dagger}\left(\begin{array}{c}
\hat{\psi}_{k+2}^{1 \dagger} \\
\hat{\psi}_{k+2}^{2 \dagger}
\end{array}\right)|0\rangle, \\
\left|\mathbf{2}_{2001}\right\rangle & \equiv \hat{\psi}_{k}^{1 \dagger} \hat{\psi}_{k}^{2 \dagger}\left(\begin{array}{c}
\hat{\psi}_{k+3}^{1 \dagger} \\
\hat{\psi}_{k+3}^{2 \dagger}
\end{array}\right)|0\rangle, \\
\left|\mathbf{2}_{0210}\right\rangle & \equiv \hat{\psi}_{k+1}^{1 \dagger} \hat{\psi}_{k+1}^{2 \dagger}\left(\begin{array}{c}
\hat{\psi}_{k+2}^{1 \dagger} \\
\hat{\psi}_{k+2}^{2 \dagger}
\end{array}\right)|0\rangle, \\
\left|\mathbf{2}_{0201}\right\rangle & \equiv \hat{\psi}_{k+1}^{1 \dagger} \hat{\psi}_{k+1}^{2 \dagger}\left(\begin{array}{c}
\hat{\psi}_{k+3}^{1 \dagger} \\
\hat{\psi}_{k+3}^{2 \dagger}
\end{array}\right)|0\rangle,
\end{aligned}
$$




$$
\begin{aligned}
\left|\mathbf{2}_{1020}\right\rangle & \equiv\left(\begin{array}{c}
\hat{\psi}_{k}^{1 \dagger} \\
\hat{\psi}_{k}^{2 \dagger}
\end{array}\right) \hat{\psi}_{k+2}^{1 \dagger} \hat{\psi}_{k+2}^{2 \dagger}|0\rangle, \\
\left|\mathbf{2}_{1002}\right\rangle & \equiv\left(\begin{array}{c}
\hat{\psi}_{k}^{1 \dagger} \\
\hat{\psi}_{k}^{2 \dagger}
\end{array}\right) \hat{\psi}_{k+3}^{1 \dagger} \hat{\psi}_{k+3}^{2 \dagger}|0\rangle, \\
\left|\mathbf{2}_{0120}\right\rangle & \equiv\left(\begin{array}{c}
\hat{\psi}_{k+1}^{1 \dagger} \\
\hat{\psi}_{k+1}^{2 \dagger}
\end{array}\right) \hat{\psi}_{k+2}^{1 \dagger} \hat{\psi}_{k+2}^{2 \dagger}|0\rangle, \\
\left|\mathbf{2}_{0102}\right\rangle & \equiv\left(\begin{array}{c}
\hat{\psi}_{k+1}^{1 \dagger} \\
\hat{\psi}_{k+1}^{2 \dagger}
\end{array}\right) \hat{\psi}_{k+3}^{1 \dagger} \hat{\psi}_{k+3}^{2 \dagger}|0\rangle, \\
\left|\mathbf{2}_{2100}\right\rangle & \equiv \hat{\psi}_{k}^{1 \dagger} \hat{\psi}_{k}^{2 \dagger}\left(\begin{array}{c}
\hat{\psi}_{k+1}^{1 \dagger} \\
\hat{\psi}_{k+1}^{2 \dagger}
\end{array}\right)|0\rangle, \\
\left|\mathbf{2}_{1200}\right\rangle & \equiv\left(\begin{array}{c}
\hat{\psi}_{k}^{1 \dagger} \\
\hat{\psi}_{k}^{2 \dagger}
\end{array}\right) \hat{\psi}_{k+1}^{1 \dagger} \hat{\psi}_{k+1}^{2 \dagger}|0\rangle,
\end{aligned}
$$

$\mathbb{H}^{\mathbf{2}^{[1110]}} \equiv\left\{\left|\mathbf{2}_{\mathrm{A}} \underline{1110}\right\rangle,\left|\mathbf{2}_{\mathrm{A}} \underline{1101}\right\rangle,\left|\mathbf{2}_{\mathrm{A} 10 \underline{11}}\right\rangle,\left|\mathbf{2}_{\mathrm{S} 10 \underline{11}}\right\rangle,\left|\mathbf{2}_{\mathrm{A} 01 \underline{11}}\right\rangle,\left|\mathbf{2}_{\mathrm{S} 01 \underline{11}}\right\rangle,\left|\mathbf{2}_{\mathrm{S} \underline{11} 10}\right\rangle,\left|\mathbf{2}_{\mathrm{S} 1101}\right\rangle\right\}$ with

$$
\begin{aligned}
\left|\mathbf{2}_{\mathrm{A} \underline{1110}}\right\rangle \equiv & \frac{1}{\sqrt{2}}\left(\hat{\psi}_{k}^{1 \dagger} \hat{\psi}_{k+1}^{2 \dagger}-\hat{\psi}_{k}^{2 \dagger} \hat{\psi}_{k+1}^{1 \dagger}\right)\left(\begin{array}{c}
\hat{\psi}_{k+2}^{1 \dagger} \\
\hat{\psi}_{k+2}^{2 \dagger}
\end{array}\right)|0\rangle, \\
\left|\mathbf{2}_{\mathrm{A} \underline{1101}}\right\rangle \equiv & \frac{1}{\sqrt{2}}\left(\hat{\psi}_{k}^{1 \dagger} \hat{\psi}_{k+1}^{2 \dagger}-\hat{\psi}_{k}^{2 \dagger} \hat{\psi}_{k+1}^{1 \dagger}\right)\left(\begin{array}{c}
\hat{\psi}_{k+3}^{1 \dagger} \\
\hat{\psi}_{k+3}^{+\dagger}
\end{array}\right)|0\rangle, \\
\left|\mathbf{2}_{\mathrm{A} 1011}\right\rangle \equiv & \frac{1}{\sqrt{2}}\left(\begin{array}{c}
\hat{\psi}_{k}^{1 \dagger} \\
\hat{\psi}_{k}^{2 \dagger}
\end{array}\right)\left(\hat{\psi}_{k+2}^{1 \dagger} \hat{\psi}_{k+3}^{2 \dagger}-\hat{\psi}_{k+2}^{2 \dagger} \hat{\psi}_{k+3}^{1 \dagger}\right)|0\rangle, \\
\left|\mathbf{2}_{\mathrm{S} 1011}\right\rangle \equiv & \left(\begin{array}{c}
\frac{1}{\sqrt{3}} \hat{\psi}_{k}^{2 \dagger} \hat{\psi}_{k+2}^{1 \dagger} \hat{\psi}_{k+3}^{1 \dagger}-\frac{1}{\sqrt{3}} \hat{\psi}_{k}^{1 \dagger}\left(\hat{\psi}_{k+2}^{1 \dagger} \hat{\psi}_{k+3}^{2 \dagger}+\hat{\psi}_{k+2}^{2 \dagger} \hat{\psi}_{k+3}^{1 \dagger}\right) \\
\frac{1}{\sqrt{6}} \hat{\psi}_{k}^{2 \dagger}\left(\hat{\psi}_{k+2}^{1 \dagger} \hat{\psi}_{k+3}^{2 \dagger}+\hat{\psi}_{k+2}^{2 \dagger} \hat{\psi}_{k+3}^{1 \dagger}\right)-\sqrt{\frac{2}{3}} \hat{\psi}_{k}^{1 \dagger} \hat{\psi}_{k+2}^{2 \dagger} \hat{\psi}_{k+3}^{2 \dagger}
\end{array}\right)|0\rangle, \\
\left|\mathbf{2}_{\mathrm{A} 0111}\right\rangle \equiv & \frac{1}{\sqrt{2}}\left(\begin{array}{l}
\hat{\psi}_{k+1}^{1 \dagger} \\
\hat{\psi}_{k+1}^{2 \dagger}
\end{array}\right)\left(\hat{\psi}_{k+2}^{1 \dagger} \hat{\psi}_{k+3}^{2 \dagger}-\hat{\psi}_{k+2}^{2 \dagger} \hat{\psi}_{k+3}^{1 \dagger}\right)|0\rangle, \\
\left|\mathbf{2}_{\mathrm{S} 0111}\right\rangle \equiv & \left(\begin{array}{c}
\frac{1}{\sqrt{3}} \hat{\psi}_{k+1}^{2 \dagger} \hat{\psi}_{k+2}^{1 \dagger} \hat{\psi}_{k+3}^{1 \dagger}-\frac{1}{\sqrt{3}} \hat{\psi}_{k+1}^{1 \dagger}\left(\hat{\psi}_{k+2}^{1 \dagger} \hat{\psi}_{k+3}^{2 \dagger}+\hat{\psi}_{k+2}^{2 \dagger} \hat{\psi}_{k+3}^{1 \dagger}\right) \\
\frac{1}{\sqrt{6}} \hat{\psi}_{k+1}^{2 \dagger}\left(\hat{\psi}_{k+2}^{1 \dagger} \hat{\psi}_{k+3}^{2 \dagger}+\hat{\psi}_{k+2}^{2 \dagger} \hat{\psi}_{k+3}^{1 \dagger}\right)-\sqrt{\frac{2}{3}} \hat{\psi}_{k+1}^{1 \dagger} \hat{\psi}_{k+2}^{2 \dagger} \hat{\psi}_{k+3}^{2 \dagger}
\end{array}\right)|0\rangle, \\
\left|\mathbf{2}_{\mathrm{S} \underline{1110}}\right\rangle \equiv & \left(\begin{array}{c}
\frac{1}{\sqrt{3}} \hat{\psi}_{k}^{1 \dagger} \hat{\psi}_{k+1}^{1 \dagger} \hat{\psi}_{k+2}^{2 \dagger}-\frac{1}{\sqrt{3}}\left(\hat{\psi}_{k}^{1 \dagger} \hat{\psi}_{k+1}^{2 \dagger}+\hat{\psi}_{k}^{2 \dagger} \hat{\psi}_{k+1}^{1 \dagger}\right) \hat{\psi}_{k+2}^{1 \dagger} \\
\frac{1}{\sqrt{6}}\left(\hat{\psi}_{k}^{1 \dagger} \hat{\psi}_{k+1}^{2 \dagger}+\hat{\psi}_{k}^{2 \dagger} \hat{\psi}_{k+1}^{1 \dagger}\right) \hat{\psi}_{k+2}^{2 \dagger}-\sqrt{\frac{2}{3}} \hat{\psi}_{k}^{2 \dagger} \hat{\psi}_{k+1}^{2 \dagger} \hat{\psi}_{k+2}^{1 \dagger}
\end{array}\right)|0\rangle, \\
\left|\mathbf{2}_{\mathrm{S} \underline{1101}}\right\rangle \equiv & \left(\begin{array}{c}
\frac{1}{\sqrt{3}} \hat{\psi}_{k}^{1 \dagger} \hat{\psi}_{k+1}^{1 \dagger} \hat{\psi}_{k+3}^{2 \dagger}-\frac{1}{\sqrt{3}}\left(\hat{\psi}_{k}^{1 \dagger} \hat{\psi}_{k+1}^{2 \dagger}+\hat{\psi}_{k}^{2 \dagger} \hat{\psi}_{k+1}^{1 \dagger}\right) \hat{\psi}_{k+3}^{1 \dagger} \\
\frac{1}{\sqrt{6}}\left(\hat{\psi}_{k}^{1 \dagger} \hat{\psi}_{k+1}^{2 \dagger}+\hat{\psi}_{k}^{2 \dagger} \hat{\psi}_{k+1}^{1 \dagger}\right) \hat{\psi}_{k+3}^{2 \dagger}-\sqrt{\frac{2}{3}} \hat{\psi}_{k}^{2 \dagger} \hat{\psi}_{k+1}^{2 \dagger} \hat{\psi}_{k+3}^{1 \dagger}
\end{array}\right)|0\rangle,
\end{aligned}
$$

with a notation A (S) for an antisymmetric (symmetric) combination in a pair of indices with the underline in the $k$-th and $(k+1)$-th vortices, or in the $(k+2)$-th and $(k+3)$-th vortices. $\mathbb{H}^{\mathbf{2}^{22210]}} \equiv$ $\left\{\left|\mathbf{2}_{2021}\right\rangle,\left|\mathbf{2}_{2012}\right\rangle,\left|\mathbf{2}_{0221}\right\rangle,\left|\mathbf{2}_{0212}\right\rangle,\left|\mathbf{2}_{2210}\right\rangle,\left|\mathbf{2}_{2201}\right\rangle,\left|\mathbf{2}_{1022}\right\rangle,\left|\mathbf{2}_{0122}\right\rangle,\left|\mathbf{2}_{2120}\right\rangle,\left|\mathbf{2}_{2102}\right\rangle,\left|\mathbf{2}_{1220}\right\rangle,\left|\mathbf{2}_{1202}\right\rangle\right\}$ with

$$
\begin{aligned}
\left|\mathbf{2}_{2021}\right\rangle & \equiv \hat{\psi}_{k}^{1 \dagger} \hat{\psi}_{k}^{2 \dagger} \hat{\psi}_{k+2}^{1 \dagger} \hat{\psi}_{k+2}^{2 \dagger}\left(\begin{array}{c}
\hat{\psi}_{k+3}^{1 \dagger} \\
\hat{\psi}_{k+3}^{2 \dagger}
\end{array}\right)|0\rangle, \\
\left|\mathbf{2}_{2012}\right\rangle & \equiv \hat{\psi}_{k}^{1 \dagger} \hat{\psi}_{k}^{2 \dagger}\left(\begin{array}{c}
\hat{\psi}_{k+2}^{1 \dagger} \\
\hat{\psi}_{k+2}^{2 \dagger}
\end{array}\right) \hat{\psi}_{k+3}^{1 \dagger} \hat{\psi}_{k+3}^{2 \dagger}|0\rangle, \\
\left|\mathbf{2}_{0221}\right\rangle & \equiv \hat{\psi}_{k+1}^{1 \dagger} \hat{\psi}_{k+1}^{2 \dagger} \hat{\psi}_{k+2}^{1 \dagger} \hat{\psi}_{k+2}^{2 \dagger}\left(\begin{array}{c}
\hat{\psi}_{k+3}^{1 \dagger} \\
\hat{\psi}_{k+3}^{2 \dagger}
\end{array}\right)|0\rangle,
\end{aligned}
$$




$$
\begin{aligned}
&\left|\mathbf{2}_{0212}\right\rangle \equiv \hat{\psi}_{k+1}^{1 \dagger} \hat{\psi}_{k+1}^{2 \dagger}\left(\begin{array}{c}
\hat{\psi}_{k+2}^{1 \dagger} \\
\hat{\psi}_{k+2}^{2 \dagger}
\end{array}\right) \hat{\psi}_{k+3}^{1 \dagger} \hat{\psi}_{k+3}^{2 \dagger}|0\rangle, \\
&\left|\mathbf{2}_{2210}\right\rangle \equiv \hat{\psi}_{k}^{1 \dagger} \hat{\psi}_{k}^{2 \dagger} \hat{\psi}_{k+1}^{1 \dagger} \hat{\psi}_{k+1}^{2 \dagger}\left(\begin{array}{c}
\hat{\psi}_{k+2}^{1 \dagger} \\
\hat{\psi}_{k+2}^{2 \dagger}
\end{array}\right)|0\rangle, \\
&\left|\mathbf{2}_{2201}\right\rangle \equiv \hat{\psi}_{k}^{1 \dagger} \hat{\psi}_{k}^{2 \dagger} \hat{\psi}_{k+1}^{1 \dagger} \hat{\psi}_{k+1}^{2 \dagger}\left(\begin{array}{c}
\hat{\psi}_{k+3}^{1 \dagger} \\
\hat{\psi}_{k+3}^{2 \dagger}
\end{array}\right)|0\rangle, \\
&\left|\mathbf{2}_{1022}\right\rangle \equiv\left(\begin{array}{c}
\hat{\psi}_{k}^{1 \dagger} \\
\hat{\psi}_{k}^{2 \dagger}
\end{array}\right) \hat{\psi}_{k+2}^{1 \dagger} \hat{\psi}_{k+2}^{2 \dagger} \hat{\psi}_{k+3}^{1 \dagger} \hat{\psi}_{k+3}^{2 \dagger}|0\rangle, \\
&\left|\mathbf{2}_{0122}\right\rangle \equiv\left(\begin{array}{c}
\hat{\psi}_{k+1}^{1 \dagger} \\
\hat{\psi}_{k+1}^{2 \dagger}
\end{array}\right) \hat{\psi}_{k+2}^{1 \dagger} \hat{\psi}_{k+2}^{2 \dagger} \hat{\psi}_{k+3}^{1 \dagger} \hat{\psi}_{k+3}^{2 \dagger}|0\rangle, \\
&\left|\mathbf{2}_{2120}\right\rangle \equiv \hat{\psi}_{k}^{1 \dagger} \hat{\psi}_{k}^{2 \dagger}\left(\begin{array}{c}
\hat{\psi}_{k+1}^{1 \dagger} \\
\hat{\psi}_{k+1}^{2 \dagger}
\end{array}\right) \hat{\psi}_{k+2}^{1 \dagger} \hat{\psi}_{k+2}^{2 \dagger}|0\rangle, \\
&\left|\mathbf{2}_{2102}\right\rangle \equiv \hat{\psi}_{k}^{1 \dagger} \hat{\psi}_{k}^{2 \dagger}\left(\begin{array}{c}
\hat{\psi}_{k+1}^{1 \dagger} \\
\hat{\psi}_{k+1}^{2 \dagger}
\end{array}\right) \hat{\psi}_{k+3}^{1 \dagger} \hat{\psi}_{k+3}^{2 \dagger}|0\rangle, \\
&\left|\mathbf{2}_{1220}\right\rangle \equiv\left(\begin{array}{c}
\hat{\psi}_{k}^{1 \dagger} \\
\hat{\psi}_{k}^{2 \dagger}
\end{array}\right) \hat{\psi}_{k+1}^{1 \dagger} \hat{\psi}_{k+1}^{2 \dagger} \hat{\psi}_{k+2}^{1 \dagger} \hat{\psi}_{k+2}^{2 \dagger}|0\rangle, \\
&\left|\mathbf{2}_{1202}\right\rangle \equiv\left(\begin{array}{c}
\hat{\psi}_{k}^{1 \dagger} \\
\hat{\psi}_{k}^{2 \dagger}
\end{array}\right) \hat{\psi}_{k+1}^{1 \dagger} \hat{\psi}_{k+1}^{2 \dagger} \hat{\psi}_{k+3}^{1 \dagger} \hat{\psi}_{k+3}^{2 \dagger}|0\rangle,
\end{aligned}
$$

$\mathbb{H}^{[2111]} \equiv\left\{\left|\mathbf{2}_{\mathrm{A}} \underline{1121}\right\rangle,\left|\mathbf{2}_{\mathrm{A}} \underline{1112}\right\rangle,\left|\mathbf{2}_{\mathrm{A} 21 \underline{11}}\right\rangle,\left|\mathbf{2}_{\mathrm{S} 21 \underline{11}}\right\rangle,\left|\mathbf{2}_{\mathrm{A} 12 \underline{11}}\right\rangle,\left|\mathbf{2}_{\mathrm{S} 12 \underline{11}}\right\rangle,\left|\mathbf{2}_{\mathrm{S}} \underline{1121}\right\rangle,\left|\mathbf{2}_{\mathrm{S} 1112}\right\rangle\right\}$ with

$$
\begin{aligned}
& \left|\mathbf{2}_{\mathrm{A}} \underline{1121}\right\rangle \equiv \frac{1}{\sqrt{2}}\left(\hat{\psi}_{k}^{1 \dagger} \hat{\psi}_{k+1}^{2 \dagger}-\hat{\psi}_{k}^{2 \dagger} \hat{\psi}_{k+1}^{1 \dagger}\right) \hat{\psi}_{k+2}^{1 \dagger} \hat{\psi}_{k+2}^{2 \dagger}\left(\begin{array}{c}
\hat{\psi}_{k+3}^{1 \dagger} \\
\hat{\psi}_{k+3}^{2 \dagger}
\end{array}\right)|0\rangle, \\
& \left|\mathbf{2}_{\mathrm{A}} \underline{1112}\right\rangle \equiv \frac{1}{\sqrt{2}}\left(\hat{\psi}_{k}^{1 \dagger} \hat{\psi}_{k+1}^{2 \dagger}-\hat{\psi}_{k}^{2 \dagger} \hat{\psi}_{k+1}^{1 \dagger}\right)\left(\begin{array}{c}
\hat{\psi}_{k+2}^{1 \dagger} \\
\hat{\psi}_{k+2}^{2 \dagger}
\end{array}\right) \hat{\psi}_{k+3}^{1 \dagger} \hat{\psi}_{k+3}^{2 \dagger}|0\rangle, \\
& \left|\mathbf{2}_{\mathrm{A} 2111}\right\rangle \equiv \frac{1}{\sqrt{2}} \hat{\psi}_{k}^{1 \dagger} \hat{\psi}_{k}^{2 \dagger}\left(\begin{array}{c}
\hat{\psi}_{k+1}^{1 \dagger} \\
\hat{\psi}_{k+1}^{2 \dagger}
\end{array}\right)\left(\hat{\psi}_{k+2}^{1 \dagger} \hat{\psi}_{k+3}^{2 \dagger}-\hat{\psi}_{k+2}^{2 \dagger} \hat{\psi}_{k+3}^{1 \dagger}\right)|0\rangle, \\
& \left|\mathbf{2}_{\mathrm{S} 2111}\right\rangle \equiv \hat{\psi}_{k}^{1 \dagger} \hat{\psi}_{k}^{2 \dagger}\left(\begin{array}{c}
\frac{1}{\sqrt{3}} \hat{\psi}_{k+1}^{2 \dagger} \hat{\psi}_{k+2}^{1 \dagger} \hat{\psi}_{k+3}^{1 \dagger}-\frac{1}{\sqrt{3}} \hat{\psi}_{k+1}^{1 \dagger}\left(\hat{\psi}_{k+2}^{1 \dagger} \hat{\psi}_{k+3}^{2 \dagger}+\hat{\psi}_{k+2}^{2 \dagger} \hat{\psi}_{k+3}^{1 \dagger}\right) \\
\frac{1}{\sqrt{6}} \hat{\psi}_{k+1}^{2 \dagger}\left(\hat{\psi}_{k+2}^{1 \dagger} \hat{\psi}_{k+3}^{2 \dagger}+\hat{\psi}_{k+2}^{2 \dagger} \hat{\psi}_{k+3}^{1 \dagger}\right)-\sqrt{\frac{2}{3}} \hat{\psi}_{k+1}^{1 \dagger} \hat{\psi}_{k+2}^{2 \dagger} \hat{\psi}_{k+3}^{2 \dagger}
\end{array}\right)|0\rangle, \\
& \left|\mathbf{2}_{\mathrm{A} 12 \underline{11}}\right\rangle \equiv \frac{1}{\sqrt{2}}\left(\begin{array}{c}
\hat{\psi}_{k}^{1 \dagger} \\
\hat{\psi}_{k}^{2 \dagger}
\end{array}\right) \hat{\psi}_{k+1}^{1 \dagger} \hat{\psi}_{k+1}^{2 \dagger}\left(\hat{\psi}_{k+2}^{1 \dagger} \hat{\psi}_{k+3}^{2 \dagger}-\hat{\psi}_{k+2}^{2 \dagger} \hat{\psi}_{k+3}^{1 \dagger}\right)|0\rangle, \\
& \left|\mathbf{2}_{\mathrm{S} 12 \underline{11}}\right\rangle \equiv \hat{\psi}_{k+1}^{1 \dagger} \hat{\psi}_{k+1}^{2 \dagger}\left(\begin{array}{c}
\frac{1}{\sqrt{3}} \hat{\psi}_{k}^{2 \dagger} \hat{\psi}_{k+2}^{1 \dagger} \hat{\psi}_{k+3}^{1 \dagger}-\frac{1}{\sqrt{3}} \hat{\psi}_{k}^{1 \dagger}\left(\hat{\psi}_{k+2}^{1 \dagger} \hat{\psi}_{k+3}^{2 \dagger}+\hat{\psi}_{k+2}^{2 \dagger} \hat{\psi}_{k+3}^{1 \dagger}\right) \\
\frac{1}{\sqrt{6}} \hat{\psi}_{k}^{2 \dagger}\left(\hat{\psi}_{k+2}^{1 \dagger} \hat{\psi}_{k+3}^{2 \dagger}+\hat{\psi}_{k+2}^{2 \dagger} \hat{\psi}_{k+3}^{1 \dagger}\right)-\sqrt{\frac{2}{3}} \hat{\psi}_{k}^{1 \dagger} \hat{\psi}_{k+2}^{2 \dagger} \hat{\psi}_{k+3}^{2 \dagger}
\end{array}\right)|0\rangle, \\
& \left|\mathbf{2}_{\mathrm{S}} \underline{1121}\right\rangle \equiv\left(\begin{array}{c}
\frac{1}{\sqrt{3}} \hat{\psi}_{k}^{1 \dagger} \hat{\psi}_{k+1}^{1 \dagger} \hat{\psi}_{k+3}^{2 \dagger}-\frac{1}{\sqrt{3}}\left(\hat{\psi}_{k}^{1 \dagger} \hat{\psi}_{k+1}^{2 \dagger}+\hat{\psi}_{k}^{2 \dagger} \hat{\psi}_{k+1}^{1 \dagger}\right) \hat{\psi}_{k+3}^{1 \dagger} \\
\frac{1}{\sqrt{6}}\left(\hat{\psi}_{k}^{1 \dagger} \hat{\psi}_{k+1}^{2 \dagger}+\hat{\psi}_{k}^{2 \dagger} \hat{\psi}_{k+1}^{1 \dagger}\right) \hat{\psi}_{k+3}^{2 \dagger}-\sqrt{\frac{2}{3}} \hat{\psi}_{k}^{2 \dagger} \hat{\psi}_{k+1}^{2 \dagger} \hat{\psi}_{k+3}^{1 \dagger}
\end{array}\right) \hat{\psi}_{k+2}^{1 \dagger} \hat{\psi}_{k+2}^{2 \dagger}|0\rangle, \\
& \left|\mathbf{2}_{\mathrm{S}} \underline{1112}\right\rangle \equiv\left(\begin{array}{c}
\frac{1}{\sqrt{3}} \hat{\psi}_{k}^{1 \dagger} \hat{\psi}_{k+1}^{1 \dagger} \hat{\psi}_{k+2}^{2 \dagger}-\frac{1}{\sqrt{3}}\left(\hat{\psi}_{k}^{1 \dagger} \hat{\psi}_{k+1}^{2 \dagger}+\hat{\psi}_{k}^{2 \dagger} \hat{\psi}_{k+1}^{1 \dagger}\right) \hat{\psi}_{k+2}^{1 \dagger} \\
\frac{1}{\sqrt{6}}\left(\hat{\psi}_{k}^{1 \dagger} \hat{\psi}_{k+1}^{2 \dagger}+\hat{\psi}_{k}^{2 \dagger} \hat{\psi}_{k+1}^{1 \dagger}\right) \hat{\psi}_{k+2}^{2 \dagger}-\sqrt{\frac{2}{3}} \hat{\psi}_{k}^{2 \dagger} \hat{\psi}_{k+1}^{2 \dagger} \hat{\psi}_{k+2}^{1 \dagger}
\end{array}\right) \hat{\psi}_{k+3}^{1 \dagger} \hat{\psi}_{k+3}^{2 \dagger}|0\rangle,
\end{aligned}
$$

with a notation A (S) for an antisymmetric (symmetric) combination in a pair of indices with the underline in the $k$-th and $(k+1)$-th vortices, or in the $(k+2)$-th and $(k+3)$-th vortices. $\mathbb{H}^{\mathbf{2}^{[2221]}} \equiv\left\{\left|\mathbf{2}_{2221}\right\rangle,\left|\mathbf{2}_{2212}\right\rangle,\left|\mathbf{2}_{2122}\right\rangle,\left|\mathbf{2}_{1222}\right\rangle\right\}$ 
with

$$
\begin{aligned}
\left|\mathbf{2}_{2221}\right\rangle & \equiv \hat{\psi}_{k}^{1 \dagger} \hat{\psi}_{k}^{2 \dagger} \hat{\psi}_{k+1}^{1 \dagger} \hat{\psi}_{k+1}^{2 \dagger} \hat{\psi}_{k+2}^{1 \dagger} \hat{\psi}_{k+2}^{2 \dagger}\left(\begin{array}{c}
\hat{\psi}_{k+3}^{1 \dagger} \\
\hat{\psi}_{k+3}^{2 \dagger}
\end{array}\right)|0\rangle, \\
\left|\mathbf{2}_{2212}\right\rangle & \equiv \hat{\psi}_{k}^{1 \dagger} \hat{\psi}_{k}^{2 \dagger} \hat{\psi}_{k+1}^{1 \dagger} \hat{\psi}_{k+1}^{2 \dagger}\left(\begin{array}{c}
\hat{\psi}_{k+2}^{1 \dagger} \\
\hat{\psi}_{k+2}^{2 \dagger}
\end{array}\right) \hat{\psi}_{k+3}^{1 \dagger} \hat{\psi}_{k+3}^{2 \dagger}|0\rangle, \\
\left|\mathbf{2}_{2122}\right\rangle & \equiv \hat{\psi}_{k}^{1 \dagger} \hat{\psi}_{k}^{2 \dagger}\left(\begin{array}{c}
\hat{\psi}_{k+1}^{1 \dagger} \\
\hat{\psi}_{k+1}^{2 \dagger}
\end{array}\right) \hat{\psi}_{k+2}^{1 \dagger} \hat{\psi}_{k+2}^{2 \dagger} \hat{\psi}_{k+3}^{1 \dagger} \hat{\psi}_{k+3}^{2 \dagger}|0\rangle, \\
\left|\mathbf{2}_{1222}\right\rangle & \equiv\left(\begin{array}{c}
\hat{\psi}_{k}^{1 \dagger} \\
\hat{\psi}_{k}^{2 \dagger}
\end{array}\right) \hat{\psi}_{k+1}^{1 \dagger} \hat{\psi}_{k+1}^{2 \dagger} \hat{\psi}_{k+2}^{1 \dagger} \hat{\psi}_{k+2}^{2 \dagger} \hat{\psi}_{k+3}^{1 \dagger} \hat{\psi}_{k+3}^{2 \dagger}|0\rangle .
\end{aligned}
$$

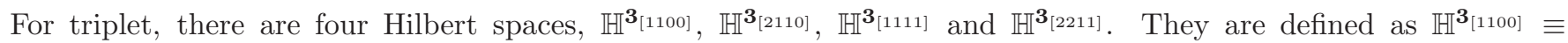
$\left\{\left|\mathbf{3}_{1100}\right\rangle,\left|\mathbf{3}_{1010}\right\rangle,\left|\mathbf{3}_{1001}\right\rangle,\left|\mathbf{3}_{0110}\right\rangle,\left|\mathbf{3}_{0101}\right\rangle,\left|\mathbf{3}_{0011}\right\rangle\right\}$ with

$$
\begin{aligned}
& \left|\mathbf{3}_{1100}\right\rangle \equiv\left(\begin{array}{c}
\hat{\psi}_{k}^{1 \dagger} \hat{\psi}_{k+1}^{1 \dagger} \\
\frac{1}{\sqrt{2}}\left(\hat{\psi}_{k}^{1 \dagger} \hat{\psi}_{k+1}^{2 \dagger}+\hat{\psi}_{k}^{2 \dagger} \hat{\psi}_{k+1}^{1 \dagger}\right) \\
\hat{\psi}_{k}^{2 \dagger} \hat{\psi}_{k+1}^{2 \dagger}
\end{array}\right)|0\rangle, \\
& \left|\mathbf{3}_{1010}\right\rangle \equiv\left(\begin{array}{c}
\hat{\psi}_{k}^{1 \dagger} \hat{\psi}_{k+2}^{1 \dagger} \\
\frac{1}{\sqrt{2}}\left(\hat{\psi}_{k}^{1 \dagger} \hat{\psi}_{k+2}^{2 \dagger}+\hat{\psi}_{k}^{2 \dagger} \hat{\psi}_{k+2}^{1 \dagger}\right) \\
\hat{\psi}_{k}^{2 \dagger} \hat{\psi}_{k+2}^{2 \dagger}
\end{array}\right)|0\rangle, \\
& \left|\mathbf{3}_{1001}\right\rangle \equiv\left(\begin{array}{c}
\hat{\psi}_{k}^{1 \dagger} \hat{\psi}_{k+3}^{1 \dagger} \\
\frac{1}{\sqrt{2}}\left(\hat{\psi}_{k}^{1 \dagger} \hat{\psi}_{k+3}^{2 \dagger}+\hat{\psi}_{k}^{2 \dagger} \hat{\psi}_{k+3}^{1 \dagger}\right) \\
\hat{\psi}_{k}^{2 \dagger} \hat{\psi}_{k+3}^{2 \dagger}
\end{array}\right)|0\rangle, \\
& \left|\mathbf{3}_{0110}\right\rangle \equiv\left(\begin{array}{c}
\hat{\psi}_{k+1}^{1 \dagger} \hat{\psi}_{k+2}^{1 \dagger} \\
\frac{1}{\sqrt{2}}\left(\hat{\psi}_{k+1}^{1 \dagger} \hat{\psi}_{k+2}^{2 \dagger}+\hat{\psi}_{k+1}^{2 \dagger} \hat{\psi}_{k+2}^{1 \dagger}\right.
\end{array}\right)|0\rangle \\
& \left|\mathbf{3}_{0101}\right\rangle \equiv\left(\begin{array}{c}
\hat{\psi}_{k+1}^{1 \dagger} \hat{\psi}_{k+3}^{1 \dagger} \\
\frac{1}{\sqrt{2}}\left(\hat{\psi}_{k+1}^{1 \dagger} \hat{\psi}_{k+3}^{2 \dagger}+\hat{\psi}_{k+1}^{2 \dagger} \hat{\psi}_{k+3}^{1 \dagger}\right) \\
\hat{\psi}_{k+1}^{2 \dagger} \hat{\psi}_{k+3}^{2 \dagger}
\end{array}\right)|0\rangle \\
& \left|\mathbf{3}_{0011}\right\rangle \equiv\left(\begin{array}{c}
\hat{\psi}_{k+2}^{1 \dagger} \hat{\psi}_{k+3}^{1 \dagger} \\
\frac{1}{\sqrt{2}}\left(\hat{\psi}_{k+2}^{1 \dagger} \hat{\psi}_{k+3}^{2 \dagger}+\hat{\psi}_{k+2}^{2 \dagger} \hat{\psi}_{k+3}^{1 \dagger}\right) \\
\hat{\psi}_{k+2}^{2 \dagger} \hat{\psi}_{k+3}^{2 \dagger}
\end{array}\right)|0\rangle
\end{aligned}
$$

$\mathbb{H}^{\left[\mathbf{3}_{[2110]}\right.} \equiv\left\{\left|\mathbf{3}_{2011}\right\rangle,\left|\mathbf{3}_{0211}\right\rangle,\left|\mathbf{3}_{1021}\right\rangle,\left|\mathbf{3}_{1012}\right\rangle,\left|\mathbf{3}_{0121}\right\rangle,\left|\mathbf{3}_{0112}\right\rangle,\left|\mathbf{3}_{2110}\right\rangle,\left|\mathbf{3}_{2101}\right\rangle,\left|\mathbf{3}_{1210}\right\rangle,\left|\mathbf{3}_{1201}\right\rangle,\left|\mathbf{3}_{1120}\right\rangle,\left|\mathbf{3}_{1102}\right\rangle\right\}$ with

$$
\begin{aligned}
& \left|\mathbf{3}_{2011}\right\rangle \equiv \hat{\psi}_{k}^{1 \dagger} \hat{\psi}_{k}^{2 \dagger}\left(\begin{array}{c}
\hat{\psi}_{k+2}^{1 \dagger} \hat{\psi}_{k+3}^{1 \dagger} \\
\frac{1}{\sqrt{2}}\left(\hat{\psi}_{k+2}^{1 \dagger} \hat{\psi}_{k+3}^{2 \dagger}+\hat{\psi}_{k+2}^{2 \dagger} \hat{\psi}_{k+3}^{1 \dagger}\right) \\
\hat{\psi}_{k+2}^{2 \dagger} \hat{\psi}_{k+3}^{2 \dagger}
\end{array}\right)|0\rangle \\
& \left|\mathbf{3}_{0211}\right\rangle \equiv \hat{\psi}_{k+1}^{1 \dagger} \hat{\psi}_{k+1}^{2 \dagger}\left(\begin{array}{c}
\hat{\psi}_{k+2}^{1 \dagger} \hat{\psi}_{k+3}^{1 \dagger} \\
\frac{1}{\sqrt{2}}\left(\hat{\psi}_{k+2}^{1 \dagger} \hat{\psi}_{k+3}^{2 \dagger}+\hat{\psi}_{k+2}^{2 \dagger} \hat{\psi}_{k+3}^{1 \dagger}\right) \\
\hat{\psi}_{k+2}^{2 \dagger} \hat{\psi}_{k+3}^{2 \dagger}
\end{array}\right)|0\rangle \\
& \left|\mathbf{3}_{1021}\right\rangle \equiv\left(\begin{array}{c}
\hat{\psi}_{k}^{1 \dagger} \hat{\psi}_{k+3}^{1 \dagger} \\
\frac{1}{\sqrt{2}}\left(\hat{\psi}_{k}^{1 \dagger} \hat{\psi}_{k+3}^{2 \dagger}+\hat{\psi}_{k}^{2 \dagger} \hat{\psi}_{k+3}^{1 \dagger}\right. \\
\hat{\psi}_{k}^{2 \dagger} \hat{\psi}_{k+3}^{2 \dagger}
\end{array}\right) \hat{\psi}_{k+2}^{1 \dagger} \hat{\psi}_{k+2}^{2 \dagger}|0\rangle, \\
& \left|\mathbf{3}_{1012}\right\rangle \equiv\left(\begin{array}{c}
\hat{\psi}_{k}^{1 \dagger} \hat{\psi}_{k+2}^{1 \dagger} \\
\frac{1}{\sqrt{2}}\left(\hat{\psi}_{k}^{1 \dagger} \hat{\psi}_{k+2}^{2 \dagger}+\hat{\psi}_{k}^{2 \dagger} \hat{\psi}_{k+2}^{1 \dagger}\right. \\
\hat{\psi}_{k}^{2 \dagger} \hat{\psi}_{k+2}^{2 \dagger}
\end{array}\right) \hat{\psi}_{k+3}^{1 \dagger} \hat{\psi}_{k+3}^{2 \dagger}|0\rangle,
\end{aligned}
$$




$$
\begin{aligned}
& \left|\mathbf{3}_{0121}\right\rangle \equiv\left(\begin{array}{c}
\hat{\psi}_{k+1}^{1 \dagger} \hat{\psi}_{k+3}^{1 \dagger} \\
\frac{1}{\sqrt{2}}\left(\hat{\psi}_{k+1}^{1 \dagger} \hat{\psi}_{k+3}^{2 \dagger}+\hat{\psi}_{k+1}^{2 \dagger} \hat{\psi}_{k+3}^{1 \dagger}\right) \\
\hat{\psi}_{k+1}^{2 \dagger} \hat{\psi}_{k+3}^{2 \dagger}
\end{array}\right) \hat{\psi}_{k+2}^{1 \dagger} \hat{\psi}_{k+2}^{2 \dagger}|0\rangle \\
& \left|\mathbf{3}_{0112}\right\rangle \equiv\left(\begin{array}{c}
\hat{\psi}_{k+1}^{1 \dagger} \hat{\psi}_{k+2}^{1 \dagger} \\
\frac{1}{\sqrt{2}}\left(\hat{\psi}_{k+1}^{1 \dagger} \hat{\psi}_{k+2}^{2 \dagger}+\hat{\psi}_{k+1}^{2 \dagger} \hat{\psi}_{k+2}^{1 \dagger}\right) \\
\hat{\psi}_{k+1}^{2 \dagger} \hat{\psi}_{k+2}^{2 \dagger}
\end{array}\right) \hat{\psi}_{k+3}^{1 \dagger} \hat{\psi}_{k+3}^{2 \dagger}|0\rangle, \\
& \left|\mathbf{3}_{2110}\right\rangle \equiv \hat{\psi}_{k}^{1 \dagger} \hat{\psi}_{k}^{2 \dagger}\left(\begin{array}{c}
\hat{\psi}_{k+1}^{1 \dagger} \hat{\psi}_{k+2}^{1 \dagger} \\
\frac{1}{\sqrt{2}}\left(\hat{\psi}_{k+1}^{1 \dagger} \hat{\psi}_{k+2}^{2 \dagger}+\hat{\psi}_{k+1}^{2 \dagger} \hat{\psi}_{k+2}^{1 \dagger}\right) \\
\hat{\psi}_{k+1}^{2 \dagger} \hat{\psi}_{k+2}^{2 \dagger}
\end{array}\right)|0\rangle \\
& \left|\mathbf{3}_{2101}\right\rangle \equiv \hat{\psi}_{k}^{1 \dagger} \hat{\psi}_{k}^{2 \dagger}\left(\begin{array}{c}
\hat{\psi}_{k+1}^{1 \dagger} \hat{\psi}_{k+3}^{1 \dagger} \\
\frac{1}{\sqrt{2}}\left(\hat{\psi}_{k+1}^{1 \dagger} \hat{\psi}_{k+3}^{2 \dagger}+\hat{\psi}_{k+1}^{2 \dagger} \hat{\psi}_{k+3}^{1 \dagger}\right) \\
\hat{\psi}_{k+1}^{2 \dagger} \hat{\psi}_{k+3}^{2 \dagger}
\end{array}\right)|0\rangle \\
& \left|\mathbf{3}_{1210}\right\rangle \equiv\left(\begin{array}{c}
\hat{\psi}_{k}^{1 \dagger} \hat{\psi}_{k+2}^{1 \dagger} \\
\frac{1}{\sqrt{2}}\left(\hat{\psi}_{k}^{1 \dagger} \hat{\psi}_{k+2}^{2 \dagger}+\hat{\psi}_{k}^{2 \dagger} \hat{\psi}_{k+2}^{1 \dagger}\right. \\
\hat{\psi}_{k}^{2 \dagger} \hat{\psi}_{k+2}^{2 \dagger}
\end{array}\right) \hat{\psi}_{k+1}^{1 \dagger} \hat{\psi}_{k+1}^{2 \dagger}|0\rangle, \\
& \left|\mathbf{3}_{1201}\right\rangle \equiv\left(\begin{array}{c}
\hat{\psi}_{k}^{1 \dagger} \hat{\psi}_{k+3}^{1 \dagger} \\
\frac{1}{\sqrt{2}}\left(\hat{\psi}_{k}^{1 \dagger} \hat{\psi}_{k+3}^{2 \dagger}+\hat{\psi}_{k}^{2 \dagger} \hat{\psi}_{k+3}^{1 \dagger}\right. \\
\hat{\psi}_{k}^{2 \dagger} \hat{\psi}_{k+3}^{2 \dagger}
\end{array}\right) \hat{\psi}_{k+1}^{1 \dagger} \hat{\psi}_{k+1}^{2 \dagger}|0\rangle, \\
& \left|\mathbf{3}_{1120}\right\rangle \equiv\left(\begin{array}{c}
\hat{\psi}_{k}^{1 \dagger} \hat{\psi}_{k+1}^{1 \dagger} \\
\frac{1}{\sqrt{2}}\left(\hat{\psi}_{k}^{1 \dagger} \hat{\psi}_{k+1}^{2 \dagger}+\hat{\psi}_{k}^{2 \dagger} \hat{\psi}_{k+1}^{1 \dagger}\right. \\
\hat{\psi}_{k}^{2 \dagger} \hat{\psi}_{k+1}^{2 \dagger}
\end{array}\right) \hat{\psi}_{k+2}^{1 \dagger} \hat{\psi}_{k+2}^{2 \dagger}|0\rangle, \\
& \left|\mathbf{3}_{1102}\right\rangle \equiv\left(\begin{array}{c}
\hat{\psi}_{k}^{1 \dagger} \hat{\psi}_{k+1}^{1 \dagger} \\
\frac{1}{\sqrt{2}}\left(\hat{\psi}_{k}^{1 \dagger} \hat{\psi}_{k+1}^{2 \dagger}+\hat{\psi}_{k}^{2 \dagger} \hat{\psi}_{k+1}^{1 \dagger}\right. \\
\hat{\psi}_{k}^{2 \dagger} \hat{\psi}_{k+1}^{2 \dagger}
\end{array}\right) \hat{\psi}_{k+3}^{1 \dagger} \hat{\psi}_{k+3}^{2 \dagger}|0\rangle
\end{aligned}
$$

$\mathbb{H}^{\mathbf{3}_{[1111]}} \equiv\left\{\left|\mathbf{3}_{\mathrm{AS}} \underline{11} \underline{11}\right\rangle,\left|\mathbf{3}_{\mathrm{SA}} \underline{11} \underline{11}\right\rangle,\left|\mathbf{3}_{\mathrm{SS}} \underline{11} \underline{11}\right\rangle\right\}$ with

$$
\begin{aligned}
& \left|\mathbf{3}_{\mathrm{AS} \underline{11} \underline{11}}\right\rangle \equiv \frac{1}{\sqrt{2}}\left(\hat{\psi}_{k}^{1 \dagger} \hat{\psi}_{k+1}^{2 \dagger}-\hat{\psi}_{k}^{2 \dagger} \hat{\psi}_{k+1}^{1 \dagger}\right)\left(\begin{array}{c}
\hat{\psi}_{k+2}^{1 \dagger} \hat{\psi}_{k+3}^{1 \dagger} \\
\frac{1}{\sqrt{2}}\left(\hat{\psi}_{k+2}^{1 \dagger} \underset{\hat{\psi}_{k+3}^{2 \dagger}+\hat{\psi}_{k+2}^{2 \dagger}}{\hat{\psi}_{k+3}^{2 \dagger}} \hat{\psi}_{k+1}^{1 \dagger}\right) \\
\hat{\psi}_{k+2}^{2 \dagger} \hat{\psi}_{k+3}^{2 \dagger}
\end{array}\right)|0\rangle, \\
& \left|\mathbf{3}_{\mathrm{SA}} \underline{11} \underline{11}\right\rangle \equiv\left(\begin{array}{c}
\hat{\psi}_{k}^{1 \dagger} \hat{\psi}_{k+1}^{1 \dagger} \\
\frac{1}{\sqrt{2}}\left(\hat{\psi}_{k}^{1 \dagger} \hat{\psi}_{k+1}^{2 \dagger}+\hat{\psi}_{k}^{2 \dagger} \hat{\psi}_{k+1}^{1 \dagger}\right) \\
\hat{\psi}_{k}^{2 \dagger} \hat{\psi}_{k+1}^{2 \dagger}
\end{array}\right) \frac{1}{\sqrt{2}}\left(\hat{\psi}_{k+2}^{1 \dagger} \hat{\psi}_{k+3}^{2 \dagger}-\hat{\psi}_{k+2}^{2 \dagger} \hat{\psi}_{k+3}^{1 \dagger}\right)|0\rangle \\
& \left|\mathbf{3}_{\mathrm{SS} \underline{11} \underline{11}}\right\rangle \equiv\left(\begin{array}{c}
\frac{1}{2} \hat{\psi}_{k}^{1 \dagger} \hat{\psi}_{k+1}^{1 \dagger}\left(\hat{\psi}_{k+2}^{1 \dagger} \hat{\psi}_{k+3}^{2 \dagger}+\hat{\psi}_{k+2}^{2 \dagger} \hat{\psi}_{k+3}^{1 \dagger}\right)-\frac{1}{2}\left(\hat{\psi}_{k}^{1 \dagger} \hat{\psi}_{k+1}^{2 \dagger}+\hat{\psi}_{k}^{2 \dagger} \hat{\psi}_{k+1}^{1 \dagger}\right) \hat{\psi}_{k+2}^{1 \dagger} \hat{\psi}_{k+3}^{1 \dagger} \\
\frac{1}{\sqrt{2}} \hat{\psi}_{k}^{1 \dagger} \hat{\psi}_{k+1}^{1 \dagger} \hat{\psi}_{k+2}^{2 \dagger} \hat{\psi}_{k+3}^{2 \dagger}-\frac{1}{\sqrt{2}} \hat{\psi}_{k}^{2 \dagger} \hat{\psi}_{k+1}^{2 \dagger} \hat{\psi}_{k+2}^{1 \dagger} \hat{\psi}_{k+3}^{1 \dagger} \\
\frac{1}{2}\left(\hat{\psi}_{k}^{1 \dagger} \hat{\psi}_{k+1}^{2 \dagger}+\hat{\psi}_{k}^{2 \dagger} \hat{\psi}_{k+1}^{1 \dagger}\right) \hat{\psi}_{k+2}^{2 \dagger} \hat{\psi}_{k+3}^{2 \dagger}-\frac{1}{2} \hat{\psi}_{k}^{2 \dagger} \hat{\psi}_{k+1}^{2 \dagger}\left(\hat{\psi}_{k+2}^{1 \dagger} \hat{\psi}_{k+3}^{2 \dagger}+\hat{\psi}_{k+2}^{2 \dagger} \hat{\psi}_{k+3}^{1 \dagger}\right)
\end{array}\right)|0\rangle
\end{aligned}
$$

with a notation $\mathrm{A}(\mathrm{S})$ for an antisymmetric (symmetric) combination in first or second pair of indices with the underline in the $k$-th and $(k+1)$-th vortices, and in the $(k+2)$-th and $(k+3)$-th vortices. $\mathbb{H}^{\mathbf{3}_{[2211]}} \equiv$ $\left\{\left|\mathbf{3}_{2211}\right\rangle,\left|\mathbf{3}_{2121}\right\rangle,\left|\mathbf{3}_{2112}\right\rangle,\left|\mathbf{3}_{1221}\right\rangle,\left|\mathbf{3}_{1212}\right\rangle,\left|\mathbf{3}_{1122}\right\rangle\right\}$ with

$$
\begin{aligned}
\left|\mathbf{3}_{2211}\right\rangle \equiv \hat{\psi}_{k}^{1 \dagger} \hat{\psi}_{k}^{2 \dagger} \hat{\psi}_{k+1}^{1 \dagger} \hat{\psi}_{k+1}^{2 \dagger}\left(\begin{array}{c}
\hat{\psi}_{k+2}^{1 \dagger} \hat{\psi}_{k+3}^{1 \dagger} \\
\frac{1}{\sqrt{2}}\left(\hat{\psi}_{k+2}^{1 \dagger} \hat{\psi}_{k+3}^{2 \dagger}+\hat{\psi}_{k+2}^{2 \dagger} \hat{\psi}_{k+3}^{1 \dagger}\right) \\
\hat{\psi}_{k+2}^{2 \dagger} \hat{\psi}_{k+3}^{2 \dagger}
\end{array}\right)|0\rangle, \\
\left|\mathbf{3}_{2121}\right\rangle \equiv \hat{\psi}_{k}^{1 \dagger} \hat{\psi}_{k}^{2 \dagger} \hat{\psi}_{k+2}^{1 \dagger} \hat{\psi}_{k+2}^{2 \dagger}\left(\begin{array}{c}
\hat{\psi}_{k+1}^{1 \dagger} \hat{\psi}_{k+3}^{1 \dagger} \\
\frac{1}{\sqrt{2}}\left(\hat{\psi}_{k+1}^{1 \dagger} \hat{\psi}_{k+3}^{2 \dagger}+\hat{\psi}_{k+1}^{2 \dagger} \hat{\psi}_{k+3}^{1 \dagger}\right) \\
\hat{\psi}_{k+1}^{2 \dagger} \hat{\psi}_{k+3}^{2 \dagger}
\end{array}\right)|0\rangle
\end{aligned}
$$




$$
\begin{aligned}
\left|\mathbf{3}_{2112}\right\rangle \equiv & \hat{\psi}_{k}^{1 \dagger} \hat{\psi}_{k}^{2 \dagger} \hat{\psi}_{k+3}^{1 \dagger} \hat{\psi}_{k+3}^{2 \dagger}\left(\begin{array}{c}
\hat{\psi}_{k+1}^{1 \dagger} \hat{\psi}_{k+2}^{1 \dagger} \\
\frac{1}{\sqrt{2}}\left(\hat{\psi}_{k+1}^{1 \dagger} \hat{\psi}_{k+2}^{2 \dagger}+\hat{\psi}_{k+1}^{2 \dagger} \hat{\psi}_{k+2}^{1 \dagger}\right) \\
\hat{\psi}_{k+1}^{2 \dagger} \hat{\psi}_{k+2}^{2 \dagger}
\end{array}\right)|0\rangle, \\
\left|\mathbf{3}_{1221}\right\rangle \equiv & \left(\begin{array}{c}
\hat{\psi}_{k}^{1 \dagger} \hat{\psi}_{k+3}^{1 \dagger} \\
\frac{1}{\sqrt{2}}\left(\begin{array}{c}
\hat{\psi}_{k}^{1 \dagger} \hat{\psi}_{k+3}^{2 \dagger}+\hat{\psi}_{k}^{2 \dagger} \hat{\psi}_{k+3}^{1 \dagger} \\
\hat{\psi}_{k}^{2 \dagger} \hat{\psi}_{k+3}^{2 \dagger}
\end{array}\right) \hat{\psi}_{k+1}^{1 \dagger} \hat{\psi}_{k+1}^{2 \dagger} \hat{\psi}_{k+2}^{1 \dagger} \hat{\psi}_{k+2}^{2 \dagger}|0\rangle, \\
\hat{\psi}_{k}^{1 \dagger} \hat{\psi}_{k+2}^{1 \dagger}
\end{array}\right) \\
\left|\mathbf{3}_{1212}\right\rangle \equiv & \left(\begin{array}{c}
\frac{1}{\sqrt{2}}\left(\begin{array}{c}
\left.\hat{\psi}_{k}^{1 \dagger} \hat{\psi}_{k+2}^{2 \dagger}+\hat{\psi}_{k}^{2 \dagger} \hat{\psi}_{k+2}^{1 \dagger}\right) \\
\hat{\psi}_{k}^{2 \dagger} \hat{\psi}_{k+2}^{2 \dagger} \\
\hat{\psi}_{k}^{1 \dagger} \hat{\psi}_{k+1}^{1 \dagger}
\end{array}\right) \hat{\psi}_{k+1}^{1 \dagger} \hat{\psi}_{k+1}^{2 \dagger} \hat{\psi}_{k+3}^{1 \dagger} \hat{\psi}_{k+3}^{2 \dagger}|0\rangle, \\
\left|\mathbf{3}_{1122}\right\rangle \equiv
\end{array}\right) \hat{\psi}_{k+2}^{1 \dagger} \hat{\psi}_{k+2}^{2 \dagger} \hat{\psi}_{k+3}^{1 \dagger} \hat{\psi}_{k+3}^{2 \dagger}|0\rangle .
\end{aligned}
$$

For quartet, there are two Hilbert spaces, $\mathbb{H}^{\mathbf{4}_{[1110]}}$ and $\mathbb{H}^{\mathbf{4}_{[2111]}}$. They are defined as $\mathbb{H}^{\mathbf{4}_{[1110]}} \equiv$ $\left\{\left|\mathbf{4}_{\mathrm{S}} \underline{1110}\right\rangle,\left|\mathbf{4}_{\mathrm{S} \underline{1101}}\right\rangle,\left|\mathbf{4}_{\mathrm{S} 10 \underline{11}}\right\rangle,\left|\boldsymbol{4}_{\mathrm{S} 01 \underline{11}}\right\rangle\right\}$ with

$$
\begin{aligned}
& \left|\mathbf{4}_{\mathrm{S} \underline{1110}}\right\rangle \equiv\left(\begin{array}{c}
\hat{\psi}_{k}^{1 \dagger} \hat{\psi}_{k+1}^{1 \dagger} \hat{\psi}_{k+2}^{1 \dagger} \\
\frac{1}{\sqrt{3}} \hat{\psi}_{k}^{1 \dagger} \hat{\psi}_{k+1}^{1 \dagger} \hat{\psi}_{k+2}^{2 \dagger}+\frac{1}{\sqrt{3}}\left(\hat{\psi} \hat{\psi}_{k}^{1 \dagger} \hat{\psi}_{k+1}^{2 \dagger}+\hat{\psi}_{k}^{2 \dagger} \hat{\psi}_{k+1}^{1 \dagger}\right) \hat{\psi}_{k+2}^{1 \dagger} \\
\frac{1}{\sqrt{3}}\left(\hat{\psi}_{k}^{1 \dagger} \hat{\psi}_{k+1}^{2 \dagger}+\hat{\psi}_{k}^{2 \dagger} \hat{\psi}_{k+1}^{1 \dagger}\right) \hat{\psi}_{k+2}^{2 \dagger}+\frac{1}{\sqrt{3}} \hat{\psi}_{k}^{2 \dagger} \hat{\psi}_{k+1}^{2 \dagger} \hat{\psi}_{k+2}^{1 \dagger} \\
\hat{\psi}_{k}^{2 \dagger} \hat{\psi}_{k+1}^{2 \dagger} \hat{\psi}_{k+2}^{2 \dagger}
\end{array}\right)|0\rangle, \\
& \left|\mathbf{4}_{\mathrm{S} \underline{1101}}\right\rangle \equiv\left(\begin{array}{c}
\hat{\psi}_{k}^{1 \dagger} \hat{\psi}_{k+1}^{1 \dagger} \hat{\psi}_{k+3}^{1 \dagger} \\
\frac{1}{\sqrt{3}} \hat{\psi}_{k}^{1 \dagger} \hat{\psi}_{k+1}^{1 \dagger} \hat{\psi}_{k+3}^{2 \dagger}+\frac{1}{\sqrt{3}}\left(\hat{\psi}_{k}^{1 \dagger} \hat{\psi}_{k+1}^{2 \dagger}+\hat{\psi}_{k}^{2 \dagger} \hat{\psi}_{k+1}^{1 \dagger}\right) \hat{\psi}_{k+3}^{1 \dagger} \\
\frac{1}{\sqrt{3}}\left(\hat{\psi}_{k}^{1 \dagger} \hat{\psi}_{k+1}^{2 \dagger}+\hat{\psi}_{k}^{2 \dagger} \hat{\psi}_{k+1}^{1 \dagger}\right) \hat{\psi}_{k+3}^{2 \dagger}+\frac{1}{\sqrt{3}} \hat{\psi}_{k}^{2 \dagger} \hat{\psi}_{k+1}^{2 \dagger} \hat{\psi}_{k+3}^{1 \dagger} \\
\hat{\psi}_{k}^{2 \dagger} \hat{\psi}_{k+1}^{2 \dagger} \hat{\psi}_{k+3}^{2 \dagger}
\end{array}\right)|0\rangle \\
& \left|\mathbf{4}_{\mathrm{S} 10 \underline{11}}\right\rangle \equiv\left(\begin{array}{c}
\hat{\psi}_{k}^{1 \dagger} \hat{\psi}_{k+2}^{1 \dagger} \hat{\psi}_{k+3}^{1 \dagger} \\
\frac{1}{\sqrt{3}} \hat{\psi}_{k}^{2 \dagger} \hat{\psi}_{k+2}^{1 \dagger} \hat{\psi}_{k+3}^{1 \dagger}+\frac{1}{\sqrt{3}} \hat{\psi}_{k}^{1 \dagger}\left(\hat{\psi}_{k+2}^{1 \dagger} \hat{\psi}_{k+3}^{2 \dagger}+\hat{\psi}_{k+2}^{2 \dagger} \hat{\psi}_{k+3}^{1 \dagger}\right) \\
\frac{1}{\sqrt{3}} \hat{\psi}_{k}^{2 \dagger}\left(\hat{\psi}_{k+2}^{1 \dagger} \hat{\psi}_{k+3}^{2 \dagger}+\hat{\psi}_{k+2}^{2 \dagger} \hat{\psi}_{k+3}^{1 \dagger}\right)+\frac{1}{\sqrt{3}} \hat{\psi}_{k}^{1 \dagger} \hat{\psi}_{k+2}^{2 \dagger} \hat{\psi}_{k+3}^{2 \dagger} \\
\hat{\psi}_{k}^{2 \dagger} \hat{\psi}_{k+2}^{2 \dagger} \hat{\psi}_{k+3}^{2 \dagger}
\end{array}\right)|0\rangle \\
& \left|\mathbf{4}_{\mathrm{S} 01 \underline{11}}\right\rangle \equiv\left(\begin{array}{c}
\hat{\psi}_{k+1}^{1 \dagger} \hat{\psi}_{k+2}^{1 \dagger} \hat{\psi}_{k+3}^{1 \dagger} \\
\frac{1}{\sqrt{3}} \hat{\psi}_{k+1}^{2 \dagger} \hat{\psi}_{k+2}^{1 \dagger} \hat{\psi}_{k+3}^{1 \dagger}+\frac{1}{\sqrt{3}} \hat{\psi}_{k+1}^{1 \dagger}\left(\hat{\psi}_{k+2}^{1 \dagger} \hat{\psi}_{k+3}^{2 \dagger}+\hat{\psi}_{k+2}^{2 \dagger} \hat{\psi}_{k+3}^{1 \dagger}\right) \\
\frac{1}{\sqrt{3}} \hat{\psi}_{k+1}^{2 \dagger}\left(\hat{\psi}_{k+2}^{1 \dagger} \hat{\psi}_{k+3}^{2 \dagger}+\hat{\psi}_{k+2}^{2 \dagger} \hat{\psi}_{k+3}^{1 \dagger}\right)+\frac{1}{\sqrt{3}} \hat{\psi}_{k+1}^{1 \dagger} \hat{\psi}_{k+2}^{2 \dagger} \hat{\psi}_{k+3}^{2 \dagger} \\
\hat{\psi}_{k+1}^{2 \dagger} \hat{\psi}_{k+2}^{2 \dagger} \hat{\psi}_{k+3}^{2 \dagger}
\end{array}\right)|0\rangle,
\end{aligned}
$$

and $\mathbb{H}^{\mathbf{4}_{[211]}} \equiv\left\{\left|\mathbf{4}_{\mathrm{S} 21 \underline{11}}\right\rangle,\left|\mathbf{4}_{\mathrm{S} 12 \underline{11}}\right\rangle,\left|\mathbf{4}_{\mathrm{S}} \underline{1121}\right\rangle,\left|\mathbf{4}_{\mathrm{S}} \underline{1112}\right\rangle\right\}$ with

$$
\begin{aligned}
& \left|\mathbf{4}_{\mathrm{S} 21 \underline{11}}\right\rangle \equiv \hat{\psi}_{k}^{1 \dagger} \hat{\psi}_{k}^{2 \dagger}\left(\begin{array}{c}
\hat{\psi}_{k+1}^{1 \dagger} \hat{\psi}_{k+2}^{1 \dagger} \hat{\psi}_{k+3}^{1 \dagger} \\
\frac{1}{\sqrt{3}} \hat{\psi}_{k+1}^{2 \dagger} \hat{\psi}_{k+2}^{1 \dagger} \hat{\psi}_{k+3}^{1 \dagger}+\frac{1}{\sqrt{3}} \hat{\psi}_{k+1}^{1 \dagger}\left(\hat{\psi}_{k+2}^{1 \dagger} \hat{\psi}_{k+3}^{2 \dagger}+\hat{\psi}_{k+2}^{2 \dagger} \hat{\psi}_{k+3}^{1 \dagger}\right) \\
\frac{1}{\sqrt{3}} \hat{\psi}_{k+1}^{2 \dagger}\left(\hat{\psi}_{k+2}^{1 \dagger} \hat{\psi}_{k+3}^{2 \dagger}+\hat{\psi}_{k+2}^{2 \dagger} \hat{\psi}_{k+3}^{1 \dagger}\right)+\frac{1}{\sqrt{3}} \hat{\psi}_{k+1}^{1 \dagger} \hat{\psi}_{k+2}^{2 \dagger} \hat{\psi}_{k+3}^{2 \dagger} \\
\hat{\psi}_{k+1}^{2 \dagger} \hat{\psi}_{k+2}^{2 \dagger} \hat{\psi}_{k+3}^{2 \dagger}
\end{array}\right)|0\rangle, \\
& \left|\mathbf{4}_{\mathrm{S} 12 \underline{11}}\right\rangle \equiv\left(\begin{array}{c}
\hat{\psi}_{k}^{1 \dagger} \hat{\psi}_{k+2}^{1 \dagger} \hat{\psi}_{k+3}^{1 \dagger} \\
\frac{1}{\sqrt{3}} \hat{\psi}_{k}^{2 \dagger} \hat{\psi}_{k+2}^{1 \dagger} \hat{\psi}_{k+3}^{1 \dagger}+\frac{1}{\sqrt{3}} \hat{\psi}_{k}^{1 \dagger}\left(\hat{\psi}_{k+2}^{1 \dagger} \hat{\psi}_{k+3}^{2 \dagger}+\hat{\psi}_{k+2}^{2 \dagger} \hat{\psi}_{k+3}^{1 \dagger}\right) \\
\frac{1}{\sqrt{3}} \hat{\psi}_{k}^{2 \dagger}\left(\hat{\psi}_{k+2}^{1 \dagger} \hat{\psi}_{k+3}^{2 \dagger}+\hat{\psi}_{k+2}^{2 \dagger} \hat{\psi}_{k+3}^{1 \dagger}\right)+\frac{1}{\sqrt{3}} \hat{\psi}_{k}^{1 \dagger} \hat{\psi}_{k+2}^{2 \dagger} \hat{\psi}_{k+3}^{2 \dagger} \\
\hat{\psi}_{k}^{2 \dagger} \hat{\psi}_{k+2}^{2 \dagger} \hat{\psi}_{k+3}^{2 \dagger}
\end{array}\right) \hat{\psi}_{k+1}^{1 \dagger} \hat{\psi}_{k+1}^{2 \dagger}|0\rangle
\end{aligned}
$$




$$
\begin{aligned}
&\left|\mathbf{4}_{\mathrm{S} \underline{1121}}\right\rangle \equiv\left(\begin{array}{c}
\hat{\psi}_{k}^{1 \dagger} \hat{\psi}_{k+1}^{1 \dagger} \hat{\psi}_{k+3}^{1 \dagger} \\
\frac{1}{\sqrt{3}} \hat{\psi}_{k}^{1 \dagger} \hat{\psi}_{k+1}^{1 \dagger} \hat{\psi}_{k+3}^{2 \dagger}+\frac{1}{\sqrt{3}}\left(\hat{\psi}_{k}^{1 \dagger} \hat{\psi}_{k+1}^{2 \dagger}+\hat{\psi}_{k}^{2 \dagger} \hat{\psi}_{k+1}^{1 \dagger}\right) \hat{\psi}_{k+3}^{1 \dagger} \\
\frac{1}{\sqrt{3}}\left(\hat{\psi}_{k}^{1 \dagger} \hat{\psi}_{k+1}^{2 \dagger}+\hat{\psi}_{k}^{2 \dagger} \hat{\psi}_{k+1}^{1 \dagger}\right) \hat{\psi}_{k+3}^{2 \dagger}+\frac{1}{\sqrt{3}} \hat{\psi}_{k}^{2 \dagger} \hat{\psi}_{k+1}^{2 \dagger} \hat{\psi}_{k+3}^{1 \dagger} \\
\hat{\psi}_{k}^{2 \dagger} \hat{\psi}_{k+1}^{2 \dagger} \hat{\psi}_{k+3}^{2 \dagger}
\end{array}\right) \hat{\psi}_{k+2}^{1 \dagger} \hat{\psi}_{k+2}^{2 \dagger}|0\rangle \\
& \hat{\psi}_{k}^{1 \dagger} \hat{\psi}_{k+1}^{1 \dagger} \hat{\psi}_{k+}^{1 \dagger} \\
&\left|\mathbf{4}_{\mathrm{S} \underline{11} 12}\right\rangle \equiv \\
&\left(\begin{array}{c}
\frac{1}{\sqrt{3}} \hat{\psi}_{k}^{1 \dagger} \hat{\psi}_{k+1}^{1 \dagger} \hat{\psi}_{k+2}^{2 \dagger}+\frac{1}{\sqrt{3}}\left(\hat{\psi}_{k}^{1 \dagger} \hat{\psi}_{k+1}^{2 \dagger}+\hat{\psi}_{k}^{2 \dagger} \hat{\psi}_{k+1}^{1 \dagger}\right) \hat{\psi}_{k+2}^{1 \dagger} \\
\frac{1}{\sqrt{3}}\left(\hat{\psi}_{k}^{1 \dagger} \hat{\psi}_{k+1}^{2 \dagger}+\hat{\psi}_{k}^{2 \dagger} \hat{\psi}_{k+1}^{1 \dagger}\right) \hat{\psi}_{k+2}^{2 \dagger}+\frac{1}{\sqrt{3}} \hat{\psi}_{k}^{2 \dagger} \hat{\psi}_{k+1}^{2 \dagger} \hat{\psi}_{k+2}^{1 \dagger} \\
\hat{\psi}_{k}^{2 \dagger} \hat{\psi}_{k+1}^{2 \dagger} \hat{\psi}_{k+2}^{2 \dagger}
\end{array}\right) \hat{\psi}_{k+3}^{1 \dagger} \hat{\psi}_{k+3}^{2 \dagger}|0\rangle
\end{aligned}
$$

with a notation $\mathrm{A}(\mathrm{S})$ for an antisymmetric (symmetric) combination in a pair of indices with the underline in the $k$-th and $(k+1)$-th vortices, or in the $(k+2)$-th and $(k+3)$-th vortices.

For quintet, there is one Hilbert space $\mathbb{H}^{\mathbf{5}_{[1111]}}$ which is defined as $\mathbb{H}^{\mathbf{5}_{[1111]}} \equiv\left\{\left|\mathbf{5}_{\mathrm{SS}} \underline{11} \underline{11}\right\rangle\right\}$ with

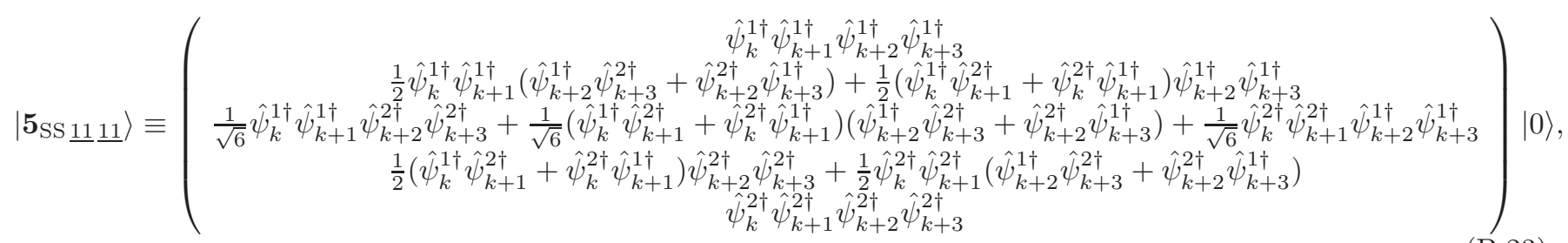

with a notation $\mathrm{S}$ for a symmetric combination in first and second pair of indices with the underline in the $k$-th and $(k+1)$-th vortices, and in the $(k+2)$-th and $(k+3)$-th vortices. Therefore, the Hilbert space is totally given as a direct sum,

$$
\begin{aligned}
\mathbb{H}^{\{n=4\}} & =\mathbb{H}^{\mathbf{1}_{[0000]}} \oplus \mathbb{H}^{\mathbf{1}_{[2000]}} \oplus \mathbb{H}^{\mathbf{1}_{[1100]}} \oplus \mathbb{H}^{\mathbf{1}_{[2200]}} \oplus \mathbb{\mathbb { H } ^ { [ 2 1 1 0 ] }} \oplus \mathbb{H}^{\mathbf{1}_{[2220]}} \oplus \mathbb{H}^{\mathbf{1}_{[1111]}} \oplus \mathbb{H}^{\mathbf{1}_{[2211]}} \oplus \mathbb{H}^{\mathbf{1}_{[222]}} \\
& \oplus \mathbb{H}^{\mathbf{2}_{[1000]}} \oplus \mathbb{H}^{\mathbf{2}_{[2100]}} \oplus \mathbb{H}^{\mathbf{2}_{[1110]}} \oplus \mathbb{H}^{\mathbf{2}_{[2210]}} \oplus \mathbb{H}^{\mathbf{2}_{[2111]}} \oplus \mathbb{H}^{\mathbf{2}_{[2221]}} \\
& \oplus \mathbb{H}^{\mathbf{3}_{[1100]}} \oplus \mathbb{H}^{\mathbf{3}_{[2110]}} \oplus \mathbb{H}^{\mathbf{3}_{[1111]}} \oplus \mathbb{H}^{\mathbf{3}_{[2211]}} \\
& \oplus \mathbb{H}^{\mathbf{4}_{[1110]}} \oplus \mathbb{H}^{\mathbf{4}_{[2111]}} \\
& \oplus \mathbb{H}^{\mathbf{5}_{[1111]}}
\end{aligned}
$$

With these basis states in the Hilbert spaces, the operators $\hat{\tau}_{k}, \hat{\tau}_{k+1}$ and $\hat{\tau}_{k+2}$ are expressed as matrices. For singlet, the matrices are

$$
\tau_{k}^{\mathbf{1}_{[0000]}}=\tau_{k+1}^{\mathbf{1}_{[0000]}}=\tau_{k+2}^{\mathbf{1}_{[0000]}}=1,
$$

for $\mathbb{H}^{\mathbf{1}_{[0000]}}$

$$
\tau_{k}^{\mathbf{1}_{[2000]}}=\left(\begin{array}{llll}
1 & 0 & 0 & 0 \\
0 & 1 & 0 & 0 \\
0 & 0 & 0 & 1 \\
0 & 0 & 1 & 0
\end{array}\right), \tau_{k+1}^{\mathbf{1}_{[2000]}}=\left(\begin{array}{llll}
0 & 0 & 0 & 1 \\
0 & 1 & 0 & 0 \\
0 & 0 & 1 & 0 \\
1 & 0 & 0 & 0
\end{array}\right), \tau_{k+2}^{\mathbf{1}_{[2000]}}=\left(\begin{array}{llll}
0 & 1 & 0 & 0 \\
1 & 0 & 0 & 0 \\
0 & 0 & 1 & 0 \\
0 & 0 & 0 & 1
\end{array}\right),
$$

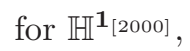

$$
\tau_{k}^{\mathbf{1}_{[1100]}}=\left(\begin{array}{cccccc}
1 & 0 & 0 & 0 & 0 & 0 \\
0 & -1 & 0 & 0 & 0 & 0 \\
0 & 0 & 0 & 0 & -1 & 0 \\
0 & 0 & 0 & 0 & 0 & -1 \\
0 & 0 & 1 & 0 & 0 & 0 \\
0 & 0 & 0 & 1 & 0 & 0
\end{array}\right), \tau_{k+1}^{\mathbf{1}_{[1100]}}=\left(\begin{array}{cccccc}
0 & 0 & 0 & 0 & 0 & 1 \\
0 & 0 & -1 & 0 & 0 & 0 \\
0 & 1 & 0 & 0 & 0 & 0 \\
0 & 0 & 0 & 1 & 0 & 0 \\
0 & 0 & 0 & 0 & -1 & 0 \\
-1 & 0 & 0 & 0 & 0 & 0
\end{array}\right), \tau_{k+2}^{\mathbf{1}_{[1100]}}=\left(\begin{array}{cccccc}
-1 & 0 & 0 & 0 & 0 & 0 \\
0 & 1 & 0 & 0 & 0 & 0 \\
0 & 0 & -1 & 0 & 0 \\
0 & 0 & 1 & 0 & 0 & 0 \\
0 & 0 & 0 & 0 & -1 \\
0 & 0 & 0 & 1 & 0
\end{array}\right),
$$


for $\mathbb{H}^{\mathbf{1}_{[1100]}}$,

$$
\tau_{k}^{\mathbf{1}_{[2200]}}=\left(\begin{array}{cccccc}
1 & 0 & 0 & 0 & 0 & 0 \\
0 & 0 & 0 & 1 & 0 & 0 \\
0 & 0 & 0 & 0 & 1 & 0 \\
0 & 1 & 0 & 0 & 0 & 0 \\
0 & 0 & 1 & 0 & 0 & 0 \\
0 & 0 & 0 & 0 & 0 & 1
\end{array}\right), \tau_{k+1}^{\mathbf{1}_{[2200]}}=\left(\begin{array}{llllll}
0 & 0 & 0 & 0 & 1 & 0 \\
0 & 0 & 0 & 0 & 0 & 1 \\
0 & 0 & 1 & 0 & 0 & 0 \\
0 & 0 & 0 & 1 & 0 & 0 \\
1 & 0 & 0 & 0 & 0 & 0 \\
0 & 1 & 0 & 0 & 0 & 0
\end{array}\right), \tau_{k+2}^{\mathbf{1}_{[2200]}}=\left(\begin{array}{llllll}
1 & 0 & 0 & 0 & 0 & 0 \\
0 & 0 & 1 & 0 & 0 & 0 \\
0 & 1 & 0 & 0 & 0 & 0 \\
0 & 0 & 0 & 0 & 1 & 0 \\
0 & 0 & 0 & 1 & 0 & 0 \\
0 & 0 & 0 & 0 & 0 & 1
\end{array}\right),
$$

for $\mathbb{H}^{\mathbf{1}_{[2200]} \text {, }}$

$$
\tau_{k}^{\mathbf{1}_{[2110]}}=\left(\begin{array}{cccccccccccc}
-1 & 0 & 0 & 0 & 0 & 0 & 0 & 0 & 0 & 0 & 0 & 0 \\
0 & -1 & 0 & 0 & 0 & 0 & 0 & 0 & 0 & 0 & 0 & 0 \\
0 & 0 & 0 & 1 & 0 & 0 & 0 & 0 & 0 & 0 & 0 & 0 \\
0 & 0 & 1 & 0 & 0 & 0 & 0 & 0 & 0 & 0 & 0 & 0 \\
0 & 0 & 0 & 0 & 0 & 0 & -1 & 0 & 0 & 0 & 0 & 0 \\
0 & 0 & 0 & 0 & 0 & 0 & 0 & -1 & 0 & 0 & 0 & 0 \\
0 & 0 & 0 & 0 & 1 & 0 & 0 & 0 & 0 & 0 & 0 & 0 \\
0 & 0 & 0 & 0 & 0 & 1 & 0 & 0 & 0 & 0 & 0 & 0 \\
0 & 0 & 0 & 0 & 0 & 0 & 0 & 0 & 0 & 0 & 1 & 0 \\
0 & 0 & 0 & 0 & 0 & 0 & 0 & 0 & 0 & 0 & 0 & 1 \\
0 & 0 & 0 & 0 & 0 & 0 & 0 & 0 & -1 & 0 & 0 & 0 \\
0 & 0 & 0 & 0 & 0 & 0 & 0 & 0 & 0 & -1 & 0 & 0
\end{array}\right), \tau_{k+1}^{\mathbf{1}_{[2110]}}=\left(\begin{array}{ccccccccccccccccc}
0 & 0 & 0 & 0 & 0 & 0 & 0 & 0 & 0 & 0 & -1 & 0 \\
0 & 0 & 0 & 0 & 0 & -1 & 0 & 0 & 0 & 0 & 0 & 0 \\
0 & 0 & 0 & 0 & 0 & 0 & 0 & 0 & 0 & 1 & 0 & 0 \\
0 & 0 & 0 & 0 & 0 & 0 & 1 & 0 & 0 & 0 & 0 & 0 \\
0 & 0 & 0 & 0 & 0 & 0 & 0 & 0 & 0 & 0 & 0 & 1 \\
0 & 1 & 0 & 0 & 0 & 0 & 0 & 0 & 0 & 0 & 0 & 0 \\
0 & 0 & 0 & -1 & 0 & 0 & 0 & 0 & 0 & 0 & 0 & 0 \\
0 & 0 & 0 & 0 & 0 & 0 & 0 & -1 & 0 & 0 & 0 & 0 \\
0 & 0 & 0 & 0 & 0 & 0 & 0 & 0 & -1 & 0 & 0 & 0 \\
0 & 0 & -1 & 0 & 0 & 0 & 0 & 0 & 0 & 0 & 0 & 0 \\
1 & 0 & 0 & 0 & 0 & 0 & 0 & 0 & 0 & 0 & 0 & 0 \\
0 & 0 & 0 & 0 & 1 & 0 & 0 & 0 & 0 & 0 & 0 & 0
\end{array}\right),
$$

for $\mathbb{H}^{\mathbf{1}_{[2110]} \text {, }}$

$$
\tau_{k}^{\mathbf{1}_{[2220]}}=\left(\begin{array}{llll}
0 & 1 & 0 & 0 \\
1 & 0 & 0 & 0 \\
0 & 0 & 1 & 0 \\
0 & 0 & 0 & 1
\end{array}\right), \tau_{k+1}^{\mathbf{1}_{[2220]}}=\left(\begin{array}{llll}
0 & 0 & 0 & 1 \\
0 & 1 & 0 & 0 \\
0 & 0 & 1 & 0 \\
1 & 0 & 0 & 0
\end{array}\right), \tau_{k+2}^{\mathbf{1}_{[2220]}}=\left(\begin{array}{llll}
1 & 0 & 0 & 0 \\
0 & 1 & 0 & 0 \\
0 & 0 & 0 & 1 \\
0 & 0 & 1 & 0
\end{array}\right),
$$

for $\mathbb{H}^{\mathbf{1}_{[2220]} \text {, }}$

$$
\tau_{k}^{\mathbf{1}_{[1111]}}=\left(\begin{array}{cc}
-1 & 0 \\
0 & 1
\end{array}\right), \tau_{k+1}^{\mathbf{1}_{[111]}}=\left(\begin{array}{cc}
\frac{1}{2} & \frac{\sqrt{3}}{2} \\
\frac{\sqrt{3}}{2} & -\frac{1}{2}
\end{array}\right), \tau_{k+2}^{\mathbf{1}_{[1111]}}=\left(\begin{array}{cc}
-1 & 0 \\
0 & 1
\end{array}\right),
$$


for $\mathbb{H}^{\mathbf{1}_{[111]}}$,

$$
\tau_{k}^{\mathbf{1}_{[2211]}}=\left(\begin{array}{cccccc}
-1 & 0 & 0 & 0 & 0 & 0 \\
0 & 1 & 0 & 0 & 0 & 0 \\
0 & 0 & 0 & 0 & 1 & 0 \\
0 & 0 & 0 & 0 & 0 & 1 \\
0 & 0 & -1 & 0 & 0 & 0 \\
0 & 0 & 0 & -1 & 0 & 0
\end{array}\right), \tau_{k+1}^{\mathbf{1}_{[2211]}}=\left(\begin{array}{cccccc}
0 & 0 & 0 & 0 & 0 & -1 \\
0 & 0 & 1 & 0 & 0 & 0 \\
0 & 1 & 0 & 0 & 0 & 0 \\
0 & 0 & 0 & -1 & 0 & 0 \\
0 & 0 & 0 & 0 & 1 & 0 \\
1 & 0 & 0 & 0 & 0 & 0
\end{array}\right), \tau_{k+2}^{\mathbf{1}_{[2211]}}=\left(\begin{array}{cccccc}
1 & 0 & 0 & 0 & 0 & 0 \\
0 & -1 & 0 & 0 & 0 & 0 \\
0 & 0 & 0 & 1 & 0 & 0 \\
0 & 0 & -1 & 0 & 0 & 0 \\
0 & 0 & 0 & 0 & 0 & 1 \\
0 & 0 & 0 & 0 & -1 & 0
\end{array}\right),
$$

for $\mathbb{H}^{\mathbf{1}_{[2211]}}$,

$$
\tau_{k}^{\mathbf{1}_{[2222]}}=\tau_{k+1}^{\mathbf{1}_{[222]}}=\tau_{k+2}^{\mathbf{1}_{[222]}}=1,
$$

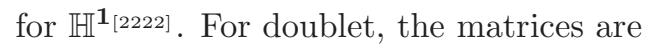

$$
\tau_{k}^{\mathbf{2}_{[1000]}}=\left(\begin{array}{cccc}
0 & -1 & 0 & 0 \\
1 & 0 & 0 & 0 \\
0 & 0 & 1 & 0 \\
0 & 0 & 0 & 1
\end{array}\right), \tau_{k+1}^{\mathbf{2}_{[1000]}}=\left(\begin{array}{cccc}
1 & 0 & 0 & 0 \\
0 & 0 & -1 & 0 \\
0 & 1 & 0 & 0 \\
0 & 0 & 0 & 1
\end{array}\right), \tau_{k+2}^{\mathbf{2}_{[1000]}}=\left(\begin{array}{cccc}
1 & 0 & 0 & 0 \\
0 & 1 & 0 & 0 \\
0 & 0 & 0 & -1 \\
0 & 0 & 1 & 0
\end{array}\right),
$$

for $\mathbb{H}^{2_{[1000]}}$,

$$
\begin{aligned}
& \tau_{k}^{\mathbf{2}_{[2100]}}=\left(\begin{array}{cccccccccccc}
1 & 0 & 0 & 0 & 0 & 0 & 0 & 0 & 0 & 0 & 0 & 0 \\
0 & 1 & 0 & 0 & 0 & 0 & 0 & 0 & 0 & 0 & 0 & 0 \\
0 & 0 & 0 & 0 & 1 & 0 & 0 & 0 & 0 & 0 & 0 & 0 \\
0 & 0 & 0 & 0 & 0 & 1 & 0 & 0 & 0 & 0 & 0 & 0 \\
0 & 0 & 1 & 0 & 0 & 0 & 0 & 0 & 0 & 0 & 0 & 0 \\
0 & 0 & 0 & 1 & 0 & 0 & 0 & 0 & 0 & 0 & 0 & 0 \\
0 & 0 & 0 & 0 & 0 & 0 & 0 & 0 & -1 & 0 & 0 & 0 \\
0 & 0 & 0 & 0 & 0 & 0 & 0 & 0 & 0 & -1 & 0 & 0 \\
0 & 0 & 0 & 0 & 0 & 0 & 1 & 0 & 0 & 0 & 0 & 0 \\
0 & 0 & 0 & 0 & 0 & 0 & 0 & 1 & 0 & 0 & 0 & 0 \\
0 & 0 & 0 & 0 & 0 & 0 & 0 & 0 & 0 & 0 & 0 & 1 \\
0 & 0 & 0 & 0 & 0 & 0 & 0 & 0 & 0 & 0 & -1 & 0
\end{array}\right), \tau_{k+1}^{\mathbf{2}_{[2100]}}=\left(\begin{array}{ccccccccccccccc}
0 & 0 & 0 & 0 & 0 & 1 & 0 & 0 & 0 & 0 & 0 & 0 \\
0 & 0 & 0 & 0 & 0 & 0 & 0 & 0 & 0 & 1 & 0 & 0 & \\
0 & 0 & 0 & 0 & 0 & 0 & 0 & 0 & 0 & 0 & 1 & 0 & \\
0 & 0 & 0 & 1 & 0 & 0 & 0 & 0 & 0 & 0 & 0 & 0 \\
0 & 0 & 0 & 0 & 0 & 0 & 0 & 0 & 1 & 0 & 0 & 0 \\
1 & 0 & 0 & 0 & 0 & 0 & 0 & 0 & 0 & 0 & 0 & 0 \\
0 & 0 & 0 & 0 & 0 & 0 & 0 & 0 & 0 & 0 & 0 & 1 \\
0 & 0 & 0 & 0 & 0 & 0 & 0 & 1 & 0 & 0 & 0 & 0 \\
0 & 0 & 0 & 0 & -1 & 0 & 0 & 0 & 0 & 0 & 0 & 0 \\
0 & -1 & 0 & 0 & 0 & 0 & 0 & 0 & 0 & 0 & 0 & 0 \\
0 & 0 & -1 & 0 & 0 & 0 & 0 & 0 & 0 & 0 & 0 & 0 \\
0 & 0 & 0 & 0 & 0 & 0 & 1 & 0 & 0 & 0 & 0 & 0
\end{array}\right), \\
& \tau_{k+2}^{\mathbf{2}_{[2100]}}=\left(\begin{array}{cccccccccccc}
0 & 1 & 0 & 0 & 0 & 0 & 0 & 0 & 0 & 0 & 0 & 0 \\
-1 & 0 & 0 & 0 & 0 & 0 & 0 & 0 & 0 & 0 & 0 & 0 \\
0 & 0 & 0 & -1 & 0 & 0 & 0 & 0 & 0 & 0 & 0 & 0 \\
0 & 0 & 1 & 0 & 0 & 0 & 0 & 0 & 0 & 0 & 0 & 0 \\
0 & 0 & 0 & 0 & 0 & -1 & 0 & 0 & 0 & 0 & 0 & 0 \\
0 & 0 & 0 & 0 & 1 & 0 & 0 & 0 & 0 & 0 & 0 & 0 \\
0 & 0 & 0 & 0 & 0 & 0 & 0 & 1 & 0 & 0 & 0 & 0 \\
0 & 0 & 0 & 0 & 0 & 0 & 1 & 0 & 0 & 0 & 0 & 0 \\
0 & 0 & 0 & 0 & 0 & 0 & 0 & 0 & 0 & 1 & 0 & 0 \\
0 & 0 & 0 & 0 & 0 & 0 & 0 & 0 & 1 & 0 & 0 & 0 \\
0 & 0 & 0 & 0 & 0 & 0 & 0 & 0 & 0 & 0 & 1 & 0 \\
0 & 0 & 0 & 0 & 0 & 0 & 0 & 0 & 0 & 0 & 0 & 1
\end{array}\right)
\end{aligned}
$$


for $\mathbb{H}^{\mathbf{2}_{[2100]} \text {, }}$

$$
\begin{aligned}
\tau_{k}^{\mathbf{2}_{[1110]}}=\left(\begin{array}{cccccccc}
-1 & 0 & 0 & 0 & 0 & 0 & 0 & 0 \\
0 & -1 & 0 & 0 & 0 & 0 & 0 & 0 \\
0 & 0 & 0 & 0 & -1 & 0 & 0 & 0 \\
0 & 0 & 0 & 0 & 0 & -1 & 0 & 0 \\
0 & 0 & 1 & 0 & 0 & 0 & 0 & 0 \\
0 & 0 & 0 & 1 & 0 & 0 & 0 & 0 \\
0 & 0 & 0 & 0 & 0 & 0 & 1 & 0 \\
0 & 0 & 0 & 0 & 0 & 0 & 0 & 1
\end{array}\right), \tau_{k+1}^{\mathbf{2}_{[1110]}}=\left(\begin{array}{ccccccccc}
\frac{1}{2} & 0 & 0 & 0 & 0 & 0 & \frac{\sqrt{3}}{2} & 0 \\
0 & 0 & \frac{1}{2} & \frac{\sqrt{3}}{2} & 0 & 0 & 0 & 0 \\
0 & -\frac{1}{2} & 0 & 0 & 0 & 0 & 0 & \frac{\sqrt{3}}{2} \\
0 & -\frac{\sqrt{3}}{2} & 0 & 0 & 0 & 0 & 0 & -\frac{1}{2} \\
0 & 0 & 0 & 0 & \frac{1}{2} & \frac{\sqrt{3}}{2} & 0 & 0 \\
0 & 0 & 0 & 0 & \frac{\sqrt{3}}{2} & -\frac{1}{2} & 0 & 0 \\
\frac{\sqrt{3}}{2} & 0 & 0 & 0 & 0 & 0 & -\frac{1}{2} & 0 \\
0 & 0 & -\frac{\sqrt{3}}{2} & \frac{1}{2} & 0 & 0 & 0 & 0
\end{array}\right), \\
\tau_{k+2}^{\mathbf{2}_{[110]}}=\left(\begin{array}{cccccccc}
0 & -1 & 0 & 0 & 0 & 0 & 0 & 0 \\
1 & 0 & 0 & 0 & 0 & 0 & 0 & 0 \\
0 & 0 & -1 & 0 & 0 & 0 & 0 & 0 \\
0 & 0 & 0 & 1 & 0 & 0 & 0 & 0 \\
0 & 0 & 0 & 0 & -1 & 0 & 0 & 0 \\
0 & 0 & 0 & 0 & 0 & 1 & 0 & 0 \\
0 & 0 & 0 & 0 & 0 & 0 & 0 & -1 \\
0 & 0 & 0 & 0 & 0 & 0 & 1 & 0
\end{array}\right),
\end{aligned}
$$

for $\mathbb{H}^{\mathbf{2}_{[1110]}}$,

$$
\begin{aligned}
& \tau_{k}^{\mathbf{2}_{[2210]}}=\left(\begin{array}{cccccccccccc}
0 & 0 & 1 & 0 & 0 & 0 & 0 & 0 & 0 & 0 & 0 & 0 \\
0 & 0 & 0 & 1 & 0 & 0 & 0 & 0 & 0 & 0 & 0 & 0 \\
1 & 0 & 0 & 0 & 0 & 0 & 0 & 0 & 0 & 0 & 0 & 0 \\
0 & 1 & 0 & 0 & 0 & 0 & 0 & 0 & 0 & 0 & 0 & 0 \\
0 & 0 & 0 & 0 & 1 & 0 & 0 & 0 & 0 & 0 & 0 & 0 \\
0 & 0 & 0 & 0 & 0 & 1 & 0 & 0 & 0 & 0 & 0 & 0 \\
0 & 0 & 0 & 0 & 0 & 0 & 0 & -1 & 0 & 0 & 0 & 0 \\
0 & 0 & 0 & 0 & 0 & 0 & 1 & 0 & 0 & 0 & 0 & 0 \\
0 & 0 & 0 & 0 & 0 & 0 & 0 & 0 & 0 & 0 & 1 & 0 \\
0 & 0 & 0 & 0 & 0 & 0 & 0 & 0 & 0 & 0 & 0 & 1 \\
0 & 0 & 0 & 0 & 0 & 0 & 0 & 0 & -1 & 0 & 0 & 0 \\
0 & 0 & 0 & 0 & 0 & 0 & 0 & 0 & 0 & -1 & 0 & 0
\end{array}\right), \tau_{k+1}^{\mathbf{2}_{[2210]}}=\left(\begin{array}{cccccccccccccc}
0 & 0 & 0 & 0 & 0 & 1 & 0 & 0 & 0 & 0 & 0 & 0 \\
0 & 0 & 0 & 0 & 0 & 0 & 0 & 0 & 0 & 1 & 0 & 0 & \\
0 & 0 & 1 & 0 & 0 & 0 & 0 & 0 & 0 & 0 & 0 & 0 & \\
0 & 0 & 0 & 0 & 0 & 0 & 0 & 1 & 0 & 0 & 0 & 0 \\
0 & 0 & 0 & 0 & 0 & 0 & 0 & 0 & 1 & 0 & 0 & 0 \\
1 & 0 & 0 & 0 & 0 & 0 & 0 & 0 & 0 & 0 & 0 & 0 \\
0 & 0 & 0 & 0 & 0 & 0 & 0 & 0 & 0 & 0 & 0 & 1 \\
0 & 0 & 0 & -1 & 0 & 0 & 0 & 0 & 0 & 0 & 0 & 0 \\
0 & 0 & 0 & 0 & -1 & 0 & 0 & 0 & 0 & 0 & 0 & 0 \\
0 & -1 & 0 & 0 & 0 & 0 & 0 & 0 & 0 & 0 & 0 & 0 \\
0 & 0 & 0 & 0 & 0 & 0 & 0 & 0 & 0 & 0 & 1 & 0 \\
0 & 0 & 0 & 0 & 0 & 0 & 1 & 0 & 0 & 0 & 0 & 0
\end{array}\right), \\
& \tau_{k+2}^{\mathbf{2}_{[2210]}}=\left(\begin{array}{cccccccccccc}
0 & 1 & 0 & 0 & 0 & 0 & 0 & 0 & 0 & 0 & 0 & 0 \\
-1 & 0 & 0 & 0 & 0 & 0 & 0 & 0 & 0 & 0 & 0 & 0 \\
0 & 0 & 0 & 1 & 0 & 0 & 0 & 0 & 0 & 0 & 0 & 0 \\
0 & 0 & -1 & 0 & 0 & 0 & 0 & 0 & 0 & 0 & 0 & 0 \\
0 & 0 & 0 & 0 & 0 & -1 & 0 & 0 & 0 & 0 & 0 & 0 \\
0 & 0 & 0 & 0 & 1 & 0 & 0 & 0 & 0 & 0 & 0 & 0 \\
0 & 0 & 0 & 0 & 0 & 0 & 1 & 0 & 0 & 0 & 0 & 0 \\
0 & 0 & 0 & 0 & 0 & 0 & 0 & 1 & 0 & 0 & 0 & 0 \\
0 & 0 & 0 & 0 & 0 & 0 & 0 & 0 & 0 & 1 & 0 & 0 \\
0 & 0 & 0 & 0 & 0 & 0 & 0 & 0 & 1 & 0 & 0 & 0 \\
0 & 0 & 0 & 0 & 0 & 0 & 0 & 0 & 0 & 0 & 0 & 1 \\
0 & 0 & 0 & 0 & 0 & 0 & 0 & 0 & 0 & 0 & 1 & 0
\end{array}\right)
\end{aligned}
$$


for $\mathbb{H}^{2^{[2210]} \text {, }}$

$$
\begin{aligned}
& \tau_{k}^{\mathbf{2}_{[2111]}}=\left(\begin{array}{cccccccc}
-1 & 0 & 0 & 0 & 0 & 0 & 0 & 0 \\
0 & -1 & 0 & 0 & 0 & 0 & 0 & 0 \\
0 & 0 & 0 & 0 & 1 & 0 & 0 & 0 \\
0 & 0 & 0 & 0 & 0 & 1 & 0 & 0 \\
0 & 0 & -1 & 0 & 0 & 0 & 0 & 0 \\
0 & 0 & 0 & -1 & 0 & 0 & 0 & 0 \\
0 & 0 & 0 & 0 & 0 & 0 & 1 & 0 \\
0 & 0 & 0 & 0 & 0 & 0 & 0 & 1
\end{array}\right), \tau_{k+1}^{\mathbf{2}_{[2111]}}=\left(\begin{array}{cccccccc}
0 & 0 & 0 & 0 & \frac{1}{2} & \frac{\sqrt{3}}{2} & 0 & 0 \\
0 & \frac{1}{2} & 0 & 0 & 0 & 0 & 0 & \frac{\sqrt{3}}{2} \\
0 & 0 & \frac{1}{2} & \frac{\sqrt{3}}{2} & 0 & 0 & 0 & 0 \\
0 & 0 & \frac{\sqrt{3}}{2} & -\frac{1}{2} & 0 & 0 & 0 & 0 \\
-\frac{1}{2} & 0 & 0 & 0 & 0 & 0 & \frac{\sqrt{3}}{2} & 0 \\
-\frac{\sqrt{3}}{2} & 0 & 0 & 0 & 0 & 0 & -\frac{1}{2} & 0 \\
0 & 0 & 0 & 0 & -\frac{\sqrt{3}}{2} & \frac{1}{2} & 0 & 0 \\
0 & \frac{\sqrt{3}}{2} & 0 & 0 & 0 & 0 & 0 & -\frac{1}{2}
\end{array}\right), \\
& \tau_{k+2}^{\mathbf{2}_{[2111]}}=\left(\begin{array}{cccccccc}
0 & 1 & 0 & 0 & 0 & 0 & 0 & 0 \\
-1 & 0 & 0 & 0 & 0 & 0 & 0 & 0 \\
0 & 0 & -1 & 0 & 0 & 0 & 0 & 0 \\
0 & 0 & 0 & 1 & 0 & 0 & 0 & 0 \\
0 & 0 & 0 & 0 & -1 & 0 & 0 & 0 \\
0 & 0 & 0 & 0 & 0 & 1 & 0 & 0 \\
0 & 0 & 0 & 0 & 0 & 0 & 0 & 1 \\
0 & 0 & 0 & 0 & 0 & 0 & -1 & 0
\end{array}\right),
\end{aligned}
$$

for $\mathbb{H}^{2^{[2111]}}$,

$$
\tau_{k}^{\mathbf{2}_{[2221]}}=\left(\begin{array}{cccc}
1 & 0 & 0 & 0 \\
0 & 1 & 0 & 0 \\
0 & 0 & 0 & 1 \\
0 & 0 & -1 & 0
\end{array}\right), \tau_{k+1}^{\mathbf{2}_{[2221]}}=\left(\begin{array}{cccc}
1 & 0 & 0 & 0 \\
0 & 0 & 1 & 0 \\
0 & -1 & 0 & 0 \\
0 & 0 & 0 & 1
\end{array}\right), \tau_{k+2}^{\mathbf{2}_{[2221]}}=\left(\begin{array}{cccc}
0 & 1 & 0 & 0 \\
-1 & 0 & 0 & 0 \\
0 & 0 & 1 & 0 \\
0 & 0 & 0 & 1
\end{array}\right),
$$

for $\mathbb{H}^{2}[2221]$. For triplet, the matrices are

$$
\tau_{k}^{\mathbf{3}_{[1100]}}=\left(\begin{array}{cccccc}
1 & 0 & 0 & 0 & 0 & 0 \\
0 & 0 & 0 & -1 & 0 & 0 \\
0 & 0 & 0 & 0 & -1 & 0 \\
0 & 1 & 0 & 0 & 0 & 0 \\
0 & 0 & 1 & 0 & 0 & 0 \\
0 & 0 & 0 & 0 & 0 & 1
\end{array}\right), \tau_{k+1}^{\mathbf{3}_{[1100]}}=\left(\begin{array}{cccccc}
0 & -1 & 0 & 0 & 0 & 0 \\
1 & 0 & 0 & 0 & 0 & 0 \\
0 & 0 & 1 & 0 & 0 & 0 \\
0 & 0 & 0 & 1 & 0 & 0 \\
0 & 0 & 0 & 0 & 0 & -1 \\
0 & 0 & 0 & 0 & 1 & 0
\end{array}\right), \tau_{k+2}^{\mathbf{3}_{[1100]}}=\left(\begin{array}{cccccc}
1 & 0 & 0 & 0 & 0 & 0 \\
0 & 0 & -1 & 0 & 0 & 0 \\
0 & 1 & 0 & 0 & 0 & 0 \\
0 & 0 & 0 & 0 & -1 & 0 \\
0 & 0 & 0 & 1 & 0 & 0 \\
0 & 0 & 0 & 0 & 0 & 1
\end{array}\right),
$$

for $\mathbb{H}^{\mathbf{3}_{[1100]}}$,

$$
\tau_{k}^{\mathbf{3}_{[2110]}}=\left(\begin{array}{cccccccccccc}
0 & 1 & 0 & 0 & 0 & 0 & 0 & 0 & 0 & 0 & 0 & 0 \\
1 & 0 & 0 & 0 & 0 & 0 & 0 & 0 & 0 & 0 & 0 & 0 \\
0 & 0 & 0 & 0 & -1 & 0 & 0 & 0 & 0 & 0 & 0 & 0 \\
0 & 0 & 0 & 0 & 0 & -1 & 0 & 0 & 0 & 0 & 0 & 0 \\
0 & 0 & 1 & 0 & 0 & 0 & 0 & 0 & 0 & 0 & 0 & 0 \\
0 & 0 & 0 & 1 & 0 & 0 & 0 & 0 & 0 & 0 & 0 & 0 \\
0 & 0 & 0 & 0 & 0 & 0 & 0 & 0 & 1 & 0 & 0 & 0 \\
0 & 0 & 0 & 0 & 0 & 0 & 0 & 0 & 0 & 1 & 0 & 0 \\
0 & 0 & 0 & 0 & 0 & 0 & -1 & 0 & 0 & 0 & 0 & 0 \\
0 & 0 & 0 & 0 & 0 & 0 & 0 & -1 & 0 & 0 & 0 & 0 \\
0 & 0 & 0 & 0 & 0 & 0 & 0 & 0 & 0 & 0 & 1 & 0 \\
0 & 0 & 0 & 0 & 0 & 0 & 0 & 0 & 0 & 0 & 0 & 1
\end{array}\right), \tau_{k+1}^{\mathbf{3}_{[2110]}}=\left(\begin{array}{cccccccccccccc}
0 & 0 & 0 & 0 & 0 & 0 & 0 & 1 & 0 & 0 & 0 & 0 \\
0 & 0 & 0 & 0 & 1 & 0 & 0 & 0 & 0 & 0 & 0 & 0 \\
0 & 0 & 0 & 0 & 0 & 0 & 0 & 0 & 0 & 1 & 0 & 0 \\
0 & 0 & 0 & 0 & 0 & 0 & 0 & 0 & 0 & 0 & 0 & 1 \\
0 & -1 & 0 & 0 & 0 & 0 & 0 & 0 & 0 & 0 & 0 & 0 \\
0 & 0 & 0 & 0 & 0 & 1 & 0 & 0 & 0 & 0 & 0 & 0 \\
0 & 0 & 0 & 0 & 0 & 0 & 1 & 0 & 0 & 0 & 0 & 0 \\
-1 & 0 & 0 & 0 & 0 & 0 & 0 & 0 & 0 & 0 & 0 & 0 \\
0 & 0 & 0 & 0 & 0 & 0 & 0 & 0 & 0 & 0 & 1 & 0 \\
0 & 0 & 1 & 0 & 0 & 0 & 0 & 0 & 0 & 0 & 0 & 0 \\
0 & 0 & 0 & 0 & 0 & 0 & 0 & 0 & -1 & 0 & 0 & 0 \\
0 & 0 & 0 & -1 & 0 & 0 & 0 & 0 & 0 & 0 & 0 & 0
\end{array}\right),
$$




$$
\tau_{k+2}^{\mathbf{3}_{[2110]}}=\left(\begin{array}{cccccccccccc}
1 & 0 & 0 & 0 & 0 & 0 & 0 & 0 & 0 & 0 & 0 & 0 \\
0 & 1 & 0 & 0 & 0 & 0 & 0 & 0 & 0 & 0 & 0 & 0 \\
0 & 0 & 0 & 1 & 0 & 0 & 0 & 0 & 0 & 0 & 0 & 0 \\
0 & 0 & -1 & 0 & 0 & 0 & 0 & 0 & 0 & 0 & 0 & 0 \\
0 & 0 & 0 & 0 & 0 & 1 & 0 & 0 & 0 & 0 & 0 & 0 \\
0 & 0 & 0 & 0 & -1 & 0 & 0 & 0 & 0 & 0 & 0 & 0 \\
0 & 0 & 0 & 0 & 0 & 0 & 0 & -1 & 0 & 0 & 0 & 0 \\
0 & 0 & 0 & 0 & 0 & 0 & 1 & 0 & 0 & 0 & 0 & 0 \\
0 & 0 & 0 & 0 & 0 & 0 & 0 & 0 & 0 & -1 & 0 & 0 \\
0 & 0 & 0 & 0 & 0 & 0 & 0 & 0 & 1 & 0 & 0 & 0 \\
0 & 0 & 0 & 0 & 0 & 0 & 0 & 0 & 0 & 0 & 0 & 1 \\
0 & 0 & 0 & 0 & 0 & 0 & 0 & 0 & 0 & 0 & 1 & 0
\end{array}\right),
$$

for $\mathbb{H}^{\mathbf{3}_{[2110]}}$

$$
\tau_{k}^{\mathbf{3}_{[1111]}}=\left(\begin{array}{ccc}
-1 & 0 & 0 \\
0 & 1 & 0 \\
0 & 0 & 1
\end{array}\right), \tau_{k+1}^{\mathbf{3}_{[111]}}=\left(\begin{array}{ccc}
\frac{1}{2} & -\frac{1}{2} & \frac{1}{\sqrt{2}} \\
-\frac{1}{2} & \frac{1}{2} & \frac{1}{\sqrt{2}} \\
\frac{1}{\sqrt{2}} & \frac{1}{\sqrt{2}} & 0
\end{array}\right), \tau_{k+2}^{\mathbf{3}_{[1111]}}=\left(\begin{array}{ccc}
1 & 0 & 0 \\
0 & -1 & 0 \\
0 & 0 & 1
\end{array}\right)
$$

for $\mathbb{H}^{\mathbf{3}_{[1111]}}$

$$
\tau_{k}^{\mathbf{3}_{[2211]}}=\left(\begin{array}{cccccc}
1 & 0 & 0 & 0 & 0 & 0 \\
0 & 0 & 0 & 1 & 0 & 0 \\
0 & 0 & 0 & 0 & 1 & 0 \\
0 & -1 & 0 & 0 & 0 & 0 \\
0 & 0 & -1 & 0 & 0 & 0 \\
0 & 0 & 0 & 0 & 0 & 1
\end{array}\right), \tau_{k+1}^{\mathbf{3}_{[2211]}}=\left(\begin{array}{cccccc}
0 & 1 & 0 & 0 & 0 & 0 \\
-1 & 0 & 0 & 0 & 0 & 0 \\
0 & 0 & 1 & 0 & 0 & 0 \\
0 & 0 & 0 & 1 & 0 & 0 \\
0 & 0 & 0 & 0 & 0 & 1 \\
0 & 0 & 0 & 0 & -1 & 0
\end{array}\right), \tau_{k+2}^{\mathbf{3}_{[2211]}}=\left(\begin{array}{cccccc}
1 & 0 & 0 & 0 & 0 & 0 \\
0 & 0 & 1 & 0 & 0 & 0 \\
0 & -1 & 0 & 0 & 0 & 0 \\
0 & 0 & 0 & 0 & 1 & 0 \\
0 & 0 & 0 & -1 & 0 & 0 \\
0 & 0 & 0 & 0 & 0 & 1
\end{array}\right),
$$

for $\mathbb{H}^{\mathbf{3}_{[2211]}}$. For quartet, the matrices are

$$
\tau_{k}^{\mathbf{4}_{[1110]}}=\left(\begin{array}{cccc}
1 & 0 & 0 & 0 \\
0 & 1 & 0 & 0 \\
0 & 0 & 0 & -1 \\
0 & 0 & 1 & 0
\end{array}\right), \tau_{k+1}^{\mathbf{4}_{[1110]}}=\left(\begin{array}{cccc}
1 & 0 & 0 & 0 \\
0 & 0 & -1 & 0 \\
0 & 1 & 0 & 0 \\
0 & 0 & 0 & 1
\end{array}\right), \tau_{k+2}^{\mathbf{4}_{[1110]}}=\left(\begin{array}{cccc}
0 & -1 & 0 & 0 \\
1 & 0 & 0 & 0 \\
0 & 0 & 1 & 0 \\
0 & 0 & 0 & 1
\end{array}\right)
$$

for $\mathbb{H}^{\mathbf{4}}[1110]$

$$
\tau_{k}^{\mathbf{4}_{[2111]}}=\left(\begin{array}{cccc}
0 & 1 & 0 & 0 \\
-1 & 0 & 0 & 0 \\
0 & 0 & 1 & 0 \\
0 & 0 & 0 & 1
\end{array}\right), \tau_{k+1}^{\mathbf{4}_{[2111]}}=\left(\begin{array}{cccc}
1 & 0 & 0 & 0 \\
0 & 0 & 1 & 0 \\
0 & -1 & 0 & 0 \\
0 & 0 & 0 & 1
\end{array}\right), \tau_{k+2}^{\mathbf{4}_{[2111]}}=\left(\begin{array}{cccc}
1 & 0 & 0 & 0 \\
0 & 1 & 0 & 0 \\
0 & 0 & 0 & 1 \\
0 & 0 & -1 & 0
\end{array}\right)
$$

for $\mathbb{H}^{\mathbf{4}_{[2111]}}$. For quintet, the matrices are

$$
\tau_{k}^{\mathbf{5}_{[1111]}}=\tau_{k+1}^{\mathbf{5}_{[1111]}}=\tau_{k+2}^{\mathbf{5}_{[1111]}}=1,
$$

for $\mathbb{H}^{\mathbf{5}_{[1111]}}$. Interestingly, we find again the non-Abelian matrices in several Hilbert subspaces. For example, the matrices $\tau_{k}^{\mathbf{1}_{[2000]}}, \tau_{k+1}^{\mathbf{1}_{[200]}}$ and $\tau_{k+2}^{\mathbf{1}_{[2000]}}$ in the Hilbert subspace $\mathbb{H}^{\mathbf{1}_{[2000]}}$ are non-commutative; $\tau_{\ell}^{\mathbf{1}_{[2000]}} \tau_{\ell+1}^{\mathbf{1}_{[200]}} \neq \tau_{\ell+1}^{\mathbf{1}_{[200]}} \tau_{\ell}^{\mathbf{1}_{[2000]}}$ for $\ell=k, k+1$. Therefore, the exchange of the $\ell$-th and $(\ell+1)$-th vortices $(\ell=k, k+1)$ induces the non-Abelian representation of the braid group in $\mathbb{H}^{\mathbf{1}_{[2000]}}$. Similarly, the non-Abelian representation of the braid group is realized

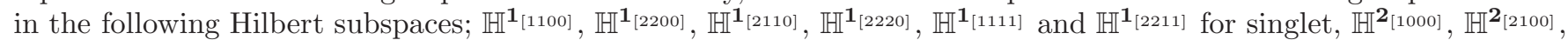

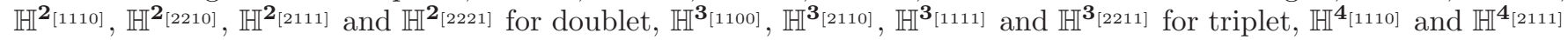
for quartet. 
As we have discussed in the text, the U(1) Dirac fermions are embedded in the U(2) Dirac fermions. When $\hat{\psi}_{\ell}^{2}$

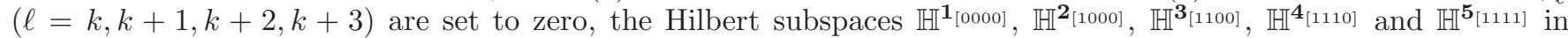
$\mathrm{U}(2)$ Dirac vortices coincide with the Hilbert subspaces $\mathbb{H}^{(4,0)}, \mathbb{H}^{(4,1)}, \mathbb{H}^{(4,2)}, \mathbb{H}^{(4,3)}$ and $\mathbb{H}^{(4,4)}$ in $\mathrm{U}(1)$ Dirac vortices, respectively. The matrices between the two are equivalent;

$$
\begin{aligned}
\tau_{\ell}^{\mathbf{1}_{[0000]}} & =\tau_{\ell}^{(4,0)}, \\
\tau_{\ell}^{\mathbf{2}_{[1000]}} & =\tau_{\ell}^{(4,1)}, \\
\tau_{\ell}^{\mathbf{3}_{[1100]}} & =\tau_{\ell}^{(4,2)}, \\
\tau_{\ell}^{\mathbf{4}_{[1110]}} & =\tau_{\ell}^{(4,3)}, \\
\tau_{\ell}^{\mathbf{5}_{[1111]}} & =\tau_{\ell}^{(4,4)},
\end{aligned}
$$

with $\ell=k, k+1, k+2$.

\section{Appendix C: Subspaces with $\left(\tau_{k}\right)^{2}=1$ in $\mathbf{U}(2)$ Dirac vortices}

We recall that, for both cases with $\mathrm{U}(1)$ and $\mathrm{U}(2)$ Dirac fermions, four-time exchange of vortices is equivalent to the identity; $\left(T_{k}\right)^{4}=1$. The same relation holds at the operator level: $\left(\hat{\tau}_{k}^{\mathrm{s}}\right)^{4}=1$ for U(1) Dirac vortices and $\left(\hat{\tau}_{k}\right)^{4}=1$ for $\mathrm{U}(2)$ Dirac vortices. However, a matrix $\tau_{k}$ representing $\hat{\tau}_{k}^{\mathrm{s}}$ or $\hat{\tau}_{k}$ happens to satisfy a stronger relation, $\left(\tau_{k}\right)^{2}=1$, in some Hilbert subspaces. There, two-time exchange of vortices is equivalent to identity. In this Appendix, we explain how such a relation can be satisfied, and check if the representation of the braid group is still non-Abelian.

First, we consider the case of U(1) Dirac fermions. From Eq. (2.9), we find that $\left(\hat{\tau}_{k}^{\mathrm{s}}\right)^{2}$ is expressed in terms of the number operator of Dirac fermions in the $\ell$-th $(\ell=k, k+1)$ vortices, $\hat{\psi}_{\ell}^{\mathrm{s \dagger}} \hat{\psi}_{\ell}^{\mathrm{s}}$. If we define $N_{\ell}^{\mathrm{s}}=0,1$ as an expectation value of the number operator $\hat{\psi}_{\ell}^{\mathrm{s} \dagger} \hat{\psi}_{\ell}^{\mathrm{s}}$, we find that the matrix $\tau_{k}^{\mathrm{s}}$ representing the operator $\hat{\tau}_{k}^{\mathrm{s}}$ yields

$$
\left(\tau_{k}^{\mathrm{s}}\right)^{2}=\left(1-2 N_{k}^{\mathrm{s}}\right)\left(1-2 N_{k+1}^{\mathrm{s}}\right) .
$$

The right-hand-side reduces to 1 only when $\left(N_{k}^{\mathrm{s}}, N_{k+1}^{\mathrm{s}}\right)=(0,0)$ or $(1,1)$. Therefore, we conclude that the relation $\left(\tau_{k}^{\mathrm{s}}\right)^{2}=1$ holds only when all the vortices are empty or fully occupied: $\left(N_{1}^{\mathrm{s}}, \cdots, N_{n}^{\mathrm{s}}\right)=(0, \cdots, 0)$ or $(1, \cdots, 1)$. In both cases, $\tau_{\ell}^{\mathrm{s}}=1$ for any $\ell=1, \cdots, n-1$, and hence it gives just trivial representation of the braid group. In fact, as shown in Appendix A, the matrices for the empty and fully-occupied states are $\tau_{\ell}^{(n, 0)}=\tau_{\ell}^{(n, n)}=1$ with $\ell=1, \cdots, n-1$ for $n=2,3,4$.

Second, let us consider the case of U(2) Dirac fermions. In this case, there are non-Abelian matrices satisfying $\left(\tau_{\ell}\right)^{2}=1$ for any $\ell=1, \cdots, n-1$. From Eq. (3.4), we find again that $\left(\hat{\tau}_{k}\right)^{2}$ is expressed in terms of the number operator of Dirac fermions in the $\ell$-th $(\ell=k, k+1)$ vortices, $\hat{\psi}_{\ell}^{a \dagger} \hat{\psi}_{\ell}^{a}$ with $a=1,2$. Note that the indices $a=1,2$ of the pseudo-spin are introduced. Then, defining $N_{\ell}^{a}=0,1$ as an expectation value of the number operator $\hat{\psi}_{\ell}^{a \dagger} \hat{\psi}_{\ell}^{a}$, we find that the matrix $\tau_{k}$ representing the operator $\hat{\tau}_{k}$ yields

$$
\left(\tau_{k}\right)^{2}=\left(1-2 N_{k}^{1}\right)\left(1-2 N_{k}^{2}\right)\left(1-2 N_{k+1}^{1}\right)\left(1-2 N_{k+1}^{2}\right) .
$$

The relation $\left(\tau_{k}\right)^{2}=1$ is fulfilled by the following combinations,

$$
\begin{array}{rllll}
\left(N_{k}^{1}, N_{k}^{2}, N_{k+1}^{1}, N_{k+1}^{2}\right)= & (0,0,0,0), & (1,1,1,1), & (1,1,0,0), & (0,0,1,1), \\
& (1,0,1,0), & (1,0,0,1), & (0,1,1,0), & (0,1,0,1) .
\end{array}
$$

When we define $N_{\ell}=N_{\ell}^{1}+N_{\ell}^{2}$, the above combinations are further rewritten as

$$
\left(N_{k}, N_{k+1}\right)=(0,0), \quad(2,2), \quad(2,0), \quad(0,2), \quad(1,1) .
$$

Therefore, we conclude that the relation $\left(\tau_{k}\right)^{2}=1$ is satisfied when $N_{k}+N_{k+1}$ is an even number. This conclusion is consistent with the expectation from the transformation properties of the Dirac fermions under the operation $\left(T_{k}\right)^{2}$. Under the two successive exchanges of $k$-th an $(k+1)$-th vortices, the Dirac fermion operators $\hat{\psi}_{k}^{a}$ and $\hat{\psi}_{k+1}^{a}$ are multiplied by -1 . If a state is composed of an even number of $k$-th and $(k+1)$-th fermions, the minus signs cancel and the state is unchanged under $\left(T_{k}\right)^{2}$. Therefore, in order for that the condition $\left(\tau_{\ell}\right)^{2}=1$ holds for any $\ell=1, \cdots, n-1$, a sum of the Dirac fermion number in the every neighboring vortices, $N_{\ell}+N_{\ell+1}$, has to be an even number. We note that $\left(N_{1}, \cdots, N_{n}\right)=(0, \cdots, 0)$ and $(2, \cdots, 2)$ corresponding to the empty state and fully-occupied 
state, respectively, give just trivial representation of the braid group $\tau_{\ell}=1$, like the case of U(1) Dirac fermions as discussed above. It is also the case for the state with the highest dimension in pseudo-spin representation. However, the other combinations of $\left(N_{1}, \cdots, N_{n}\right)$ induce non-Abelian matrices, namely non-Abelian representation of the braid group.

Let us see examples in $\mathrm{U}(2)$ Dirac fermions with $n=3$ and 4 .

a) For $n=3$, the Hilbert subspaces, in which $N_{\ell}+N_{\ell+1}$ is an even number for any $\ell=1,2$, are

$$
\mathbb{H}^{\mathbf{1}_{[000]},} \quad \mathbb{H}^{\mathbf{1}_{[200]},} \quad \mathbb{H}^{\mathbf{1}_{[220]},} \quad \mathbb{H}^{\mathbf{1}_{[222]},} \quad \mathbb{H}^{\mathbf{2}_{[111]}} \text { and } \mathbb{H}^{\mathbf{4}_{[111]}} \text {. }
$$

The matrices in each Hilbert subspace are

$$
\tau_{\ell}^{\mathbf{1}_{[000]}}, \quad \tau_{\ell}^{\mathbf{1}_{[200]}}, \quad \tau_{\ell}^{\mathbf{1}_{[220]}}, \quad \tau_{\ell}^{\mathbf{1}_{[222]}}, \quad \tau_{\ell}^{\mathbf{2}_{[111]}} \text { and } \tau_{\ell}^{\mathbf{4}_{[111]}} .
$$

Among them, the matrices $\tau_{\ell}^{\mathbf{1}_{[000]}}, \tau_{\ell}^{\mathbf{1}_{[22]}}$ and $\tau_{\ell}^{\mathbf{4}_{[111]}}$ are trivial, because they correspond to the empty state, fulloccupied state and the state with highest dimension in pseudo-spin representation, respectively. The other matrices $\tau_{\ell}^{\mathbf{1}_{[200]}}, \tau_{\ell}^{\mathbf{1}_{[220]}}$ and $\tau_{\ell}^{\mathbf{2}_{[111]}}$ are non-Abelian matrices, and hence the Hilbert subspaces $\mathbb{H}^{\mathbf{1}_{[200]}}, \mathbb{H}^{\mathbf{1}_{[220]}}$ and $\mathbb{H}^{\mathbf{2}_{[111]}}$ lead to the non-Abelian representation of the braid group satisfying $\left(\tau_{\ell}\right)^{2}=1$ for $\ell=1,2$.

b) For $n=4$, from Appendix B] the Hilbert subspaces, in which $N_{\ell}+N_{\ell+1}$ is an even number for any $\ell=1,2,3$, are

$$
\mathbb{H}^{\mathbf{1}_{[0000]}}, \quad \mathbb{H}^{\mathbf{1}_{[2000]},} \quad \mathbb{H}^{\mathbf{1}_{[2200]}}, \quad \mathbb{H}^{\mathbf{1}_{[2220]},} \quad \mathbb{H}^{\mathbf{1}_{[1111]},} \quad \mathbb{H}^{\mathbf{1}_{[2222]}}, \quad \mathbb{H}^{\mathbf{3}_{[1111]}} \quad \text { and } \quad \mathbb{H}^{\mathbf{5}_{[1111]}}
$$

The matrices in each Hilbert subspace are

$$
\tau_{\ell}^{\mathbf{1}_{[0000]}}, \quad \tau_{\ell}^{\mathbf{1}_{[2000]}}, \quad \tau_{\ell}^{\mathbf{1}_{[2200]}}, \quad \tau_{\ell}^{\mathbf{1}_{[2220]}}, \quad \tau_{\ell}^{\mathbf{1}_{[1111]}}, \quad \tau_{\ell}^{\mathbf{1}_{[2222]}}, \quad \tau_{\ell}^{\mathbf{3}_{[1111]}} \text { and } \tau_{\ell}^{\mathbf{5}_{[1111]}} \text {. }
$$

Among them, the matrices $\tau_{\ell}^{\mathbf{1}_{[0000]}}, \tau_{\ell}^{\mathbf{1}_{[222]}}$ and $\tau_{\ell}^{\mathbf{5}_{[1111]}}$ are trivial, because they correspond to the empty state, fulloccupied state and the state with highest dimension in pseudo-spin representation, respectively. The other matrices

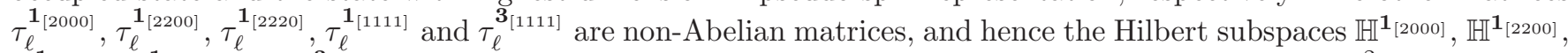
$\mathbb{H}^{\mathbf{1}_{[220]}}, \mathbb{H}^{\mathbf{1}_{[1111]}}$ and $\mathbb{H}^{\mathbf{3}_{[1111]}}$ lead to the non-Abelian representation of the braid group satisfying $\left(\tau_{\ell}\right)^{2}=1$ for $\ell=1$, 2,3 .

We note that the condition $\left(\tau_{k}\right)^{2}=1$ with the braid relations (i) $\tau_{k} \tau_{\ell} \tau_{k}=\tau_{\ell} \tau_{k} \tau_{\ell}$ for $|k-\ell|=1$ and (ii) $\tau_{k} \tau_{\ell}=\tau_{\ell} \tau_{k}$ for $|k-\ell|>1$ leads to the relations, (i') $\left(\tau_{k} \tau_{\ell}\right)^{3}=1$ for $|k-\ell|=1$ and (ii') $\left(\tau_{k} \tau_{\ell}\right)^{2}=1$ for $|k-\ell|>1$ for $k, \ell=1, \cdots, n-1$. The relations (i') and (ii') imply that such matrices $\tau_{\ell}$ are regarded as generators of the symmetric group or the Coxeter group of the type $A_{n-1}$. We recall that the Coxeter group was obtained in the $\mathrm{SO}(3)$ or more generally $\mathrm{SO}(N)$ ( $N$ odd numbers) symmetric Majorana vortices [8, 10]. There, the matrices for exchanging vortices were tensor product of "the Ivanov matrices" found by Ivanov [6] and the generators of the Coxeter group of $A_{2 m-1}$ type ( $2 m$ the number of vortices). In the present case of U(2) Dirac vortices, however, such tensor structure was not found.

[1] A. Schnyder, S. Ryu, A. Furusaki, and A. Ludwig, Classification of topological insulators and superconductors in three spatial dimensions, Phys. Rev. B 78 (2008) 195125 arXiv:0803.2786 [cond-mat.mes-hall]]; Classification of Topological Insulators and Superconductors, AIP Conf. Proc. 1134 (2009) 10 arXiv:0905.2029 [cond-mat.mes-hall]].

[2] A. Kitaev, Periodic table for topological insulators and superconductors, AIP Conf. Proc. 1134 (2009) 22 [arXiv:0901.2686 [cond-mat.mes-hall]].

[3] G. E. Volovik, Fermion zero modes on vortices in chiral superconductors, JETP Lett. 70 (1999) 609-614 arXiv:cond-mat/9909426.

[4] R. Roy, Topological Majorana and Dirac Zero Modes in Superconducting Vortex Cores, Phys. Rev. Lett. 105 (2010) 186401 arXiv:1001.2571 [cond-mat.supr-con]].

[5] N. Read and D. Green, Paired states of fermions in two-dimensions with breaking of parity and time reversal symmetries, and the fractional quantum Hall effect, Phys. Rev. B 61 (2000) 10267 arXiv:cond-mat/9906453.

[6] D. A. Ivanov, Non-Abelian Statistics of Half-Quantum Vortices in p-Wave Superconductors, Phys. Rev. Lett. 86 (2001) 268 arXiv:cond-mat/0005069 [cond-mat.supr-con]].

[7] F. Wilczek, Majorana returns, Nature Phys. 5 (2009) 614.

[8] S. Yasui, K. Itakura and M. Nitta, Majorana meets Coxeter: Non-Abelian Majorana Fermions and Non-Abelian Statistics, Phys. Rev. B 83 (2011) 134518 arXiv:1010.3331 [cond-mat.mes-hall]].

[9] S. Yasui, K. Itakura and M. Nitta, Fermion structure of non-Abelian vortices in high density QCD, Phys. Rev. D 81 (2010) 105003 arXiv:1001.3730 [hep-ph]]. 
[10] Y. Hirono, S. Yasui, K. Itakura and M. Nitta, Non-Abelian statistics of vortices with multiple Majorana fermions, arXiv:1203.0173 [cond-mat.supr-con]].

[11] S. Yasui, K. Itakura and M. Nitta, Dirac returns: Non-Abelian statistics of vortices with Dirac fermions, Nucl. Phys. B 859 (2012) 261-268 arXiv:1109.2755 [cond-mat.supr-con]].

[12] J. C. Y. Teo and C. L. Kane, Topological defects and gapless modes in insulators and superconductors, Phys. Rev. B 82 (2010) 115120.

[13] E. Majorana, Theory Of The Symmetry Of Electrons And Positrons, Nuovo Cim. 14 (1937) 171.

[14] A. P. Mackenzie and Y. Maeno, The superconductivity of $\mathrm{Sr}_{2} \mathrm{RuO}_{4}$ and the physics of spin-triplet pairing, Rev. Mod. Phys. 75 (2003) 657.

[15] M. Sato, Non-Abelian statistics of axion strings, Phys. Lett. B 575 (2003) 126.

[16] L. Fu and C. L. Kane, Superconducting Proximity Effect and Majorana Fermions at the Surface of a Topological Insulator, Phys. Rev. Lett. 100 (2008) 096407.

[17] T. Kawakami, Y. Tsutsumi and K. Machida, Singular and Half-Quantum Vortices and Associated Majorana Particles in Superfluid ${ }^{3}$ He-A between Parallel Plates, J. Phys. Soc. Jpn. 79 (2010) 044607 arXiv:0912.3085 [cond-mat.supr-con]]; T. Kawakami, T. Mizushima and K. Machida, Zero Energy Modes and Statistics of Vortices in Spinful Chiral p-Wave Superfluids, J. Phys. Soc. Jpn. 80 (2011) 044603 arXiv:1010.5038 [cond-mat.supr-con]].

[18] N. B. Kopnin and M. M. Salomaa, Mutual friction in superfluid ${ }^{3}$ He: Effects of bound states in the vortex core, Phys. Rev. B 44 (1991) 9667.

[19] K. I. Imura, Y. Takane and A. Tanaka, Weak topological insulator with protected gapless helical states, Phys. Rev. B 84 (2011) 035443 arXiv:1103.1430 [cond-mat.mes-hall]].

[20] R. Jackiw and P. Rossi, Zero Modes Of The Vortex - Fermion System, Nucl. Phys. B 190 (1981) 681.

[21] A. P. Balachandran, S. Digal and T. Matsuura, Semi-superfluid strings in high density QCD, Phys. Rev. D 73 (2006) 074009 hep-ph/0509276.

[22] M. G. Alford, A. Schmitt, K. Rajagopal, T. Schafer, Color superconductivity in dense quark matter, Rev. Mod. Phys. 80 (2008) 1455 arXiv:0709.4635 [hep-ph]].

[23] E. Nakano, M. Nitta and T. Matsuura, Non-Abelian Strings in High Density QCD: Zero Modes and Interactions, Phys. Rev. D 78, 045002 (2008) arXiv:0708.4096 [hep-ph]]; Non-Abelian Strings in Hot or Dense QCD, Prog. Theor. Phys. Suppl. 174 (2008) 254 arXiv:0805.4539 [hep-ph]]; M. Eto and M. Nitta, Color Magnetic Flux Tubes in Dense QCD, Phys. Rev. D 80 (2009) 125007 arXiv:0907.1278 [hep-ph]]; M. Eto, E. Nakano and M. Nitta, Effective World-Sheet Theory Of Color Magnetic Flux Tubes In Dense QCD, Phys. Rev. D 80 (2009) 125011 arXiv:0908.4470 [hep-ph]]; M. Eto, M. Nitta and N. Yamamoto, Instabilities of Non-Abelian Vortices in Dense QCD, Phys. Rev. Lett. 104 (2010) 161601 arXiv:0912.1352 [hep-ph]]; Y. Hirono, T. Kanazawa and M. Nitta, Topological Interactions of Non-Abelian Vortices with Quasi-Particles in High Density QCD, Phys. Rev. D 83 (2011) 085018 arXiv:1012.6042 [hep-ph]]; M. Eto, M. Nitta and N. Yamamoto, Confined Monopoles Induced by Quantum Effects in Dense QCD, Phys. Rev. D 83 (2011) 085005 arXiv:1101.2574 [hep$\mathrm{ph}]$.

[24] T. Fujiwara, T. Fukui, M. Nitta and S. Yasui, Index theorem and Majorana zero modes along a non-Abelian vortex in a color superconductor, Phys. Rev. D 84 (2011) 076002 arXiv:1105.2115 [hep-ph]].

[25] A. Hanany and D. Tong, Vortices, instantons and branes, JHEP 0307 (2003) 037 arXiv:hep-th/0306150]; R. Auzzi, S. Bolognesi, J. Evslin, K. Konishi and A. Yung, Nonabelian superconductors: Vortices and confinement in N $=2$ SQCD, Nucl. Phys. B 673 (2003) 187 arXiv:hep-th/0307287]; M. Eto, Y. Isozumi, M. Nitta, K. Ohashi and N. Sakai, Moduli space of non-Abelian vortices, Phys. Rev. Lett. 96 (2006) 161601 hep-th/0511088.

[26] D. Tong, TASI lectures on solitons: Instantons, monopoles, vortices and kinks, arXiv:hep-th/0509216]; M. Eto, Y. Isozumi, M. Nitta, K. Ohashi and N. Sakai, Solitons in the Higgs phase: The moduli matrix approach, J. Phys. A 39 R315 (2006) arXiv:hep-th/0602170; D. Tong, Quantum Vortex Strings: A Review, Annals Phys. 324 (2009) 30 arXiv:0809.5060 [hepth]]; M. Shifman and A. Yung, Supersymmetric Solitons and How They Help Us Understand Non-Abelian Gauge Theories, Rev. Mod. Phys. 79 (2007) 1139 arXiv:hep-th/0703267; an expanded version in Cambridge University Press, 2009.

[27] A. Kitaev, Anyons in an exactly solved model and beyond, Ann. Phys. 321 (2006) 2 arXiv:cond-mat/0506438 [condmat.mes-hall]].

[28] For a review, see C. Nayak, S. H. Simon, A. Stern, M. Freedman and S. Das Sarma, Non-Abelian anyons and topological quantum computation, Rev. Mod. Phys. 80 (2008) 1083 [arXiv:0707.1889 [cond-mat.str-el]].

[29] In the previous paper for the U(1) Dirac case [11], we used the word "non-Abelian statistics" to imply non-Abelian representations of the braid group. However, in the present paper, we use the word "non-Abelian statistics" only for exchanges of two identical states, which should be a subgroup of the whole representation. 